\title{
The Vienna-KPNO search for Doppler-imaging candidate stars
}

\section{A catalog of stellar-activity indicators for 1058 late-type Hipparcos stars ${ }^{\star}$}

\author{
K.G. Strassmeier ${ }^{\star \star}$, A. Washuettl ${ }^{\star \star}$, Th. Granzer ${ }^{\star \star}$, M. Scheck, and M. Weber ${ }^{\star \star}$ \\ Institut für Astronomie, Universität Wien, Türkenschanzstraße 17, A-1180 Wien, Austria \\ e-mail: name@astro.univie.ac.at
}

Received November 2; accepted December 22, 1999

\begin{abstract}
We present the results from a spectroscopic Ca II $\mathrm{H} \& \mathrm{~K}$ survey of 1058 late-type stars selected from a colorlimited subsample of the Hipparcos catalog. Out of these 1058 stars, 371 stars were found to show significant H\&K emission, most of them previously unknown; $23 \%$ with strong emission, $36 \%$ with moderate emission, and $41 \%$ with weak emission. These spectra are used to determine absolute H\&K emission-line fluxes, radial velocities, and equivalent widths of the luminosity-sensitive Sr II line at $4077 \AA$ A. Red-wavelength spectroscopic and Strömgren $y$ photometric follow-up observations of the 371 stars with $H \& \mathrm{~K}$ emission are used to additionally determine the absolute $\mathrm{H} \alpha$-core flux, the lithium abundance from the Li I 6708 A equivalent width, the rotational velocity $v \sin i$, the radial velocity, and the light variations and its periodicity. The latter is interpreted as the stellar rotation period due to an inhomogeneous surface brightness distribution. 156 stars were found with photometric periods between 0.29 and 64 days, 11 additional systems showed quasi-periodic variations possibly in excess of $\approx 50$ days. Further 54 stars had variations but no unique period was found, and four stars were essentially constant. Altogether, 170 new variable stars were discovered. Additionally, we found 17 new SB1 (plus 16 new candidates) and 19 new SB2 systems, as well as one definite and two possible new SB3 systems. Finally, we present a list of 21 stars that we think are most suitable candidates for a detailed study with the Doppler-imaging technique.
\end{abstract}

Key words: stars: activity — stars: chromospheres — stars: late-type - stars: rotation — surveys

\footnotetext{
* Tables A1-A3 are only available in electronic form at the CDS via anonymous ftp to cdsarc.u-strasbg.fr (130.79.128.5) or via http://cdsweb.u-strasbg.fr/Abstract.html

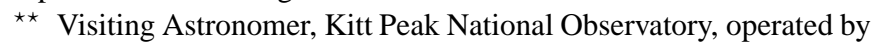
the Association of Universities for Research in Astronomy, Inc. under contract with the National Science Foundation.
}

\section{Scientific motivation for a Ca II H\&K survey}

The presence of emission in the core of the $\mathrm{Ca}$ II $\mathrm{H}$ and $\mathrm{K}$ resonance lines is a diagnostic of magnetic activity in the chromospheres of late-type stars. Spatially resolved K-line heliograms and magnetograms amply demonstrate the relation between $\mathrm{H} \& \mathrm{~K}$-emission strength and the surface magnetic field on our Sun (Schrijver 1996). Furthermore, the fact that we observe generally stronger $\mathrm{H} \& \mathrm{~K}$ emission in more rapidly rotating stars is widely known as the rotation-activity relation (e.g. Noyes et al. 1984) which is heuristically explained by the $\Omega$ effect of the classic $\alpha \Omega$ dynamo (see Stix 1989). Therefore, rapidly-rotating stars offer laboratories to study the effect of stellar dynamos. The catalog of chromospherically active binary stars (CABS, Strassmeier et al. 1993) summarized such stars in binaries and proofed to be a valuable data base for further investigations.

It is only the very rapidly-rotating stars where we can also obtain spatially resolved information of their surface temperature distribution, and respectively also of their magnetic surface field, by applying indirect imaging techniques like Doppler imaging (e.g. Rice 1996). Such rapidly-rotating active stars are relatively rare but can be identified from their $\mathrm{Ca}$ II H\&K emission with just a single spectrum of low signal-to-noise ratio and moderate resolution. For example, a H\&K survey from low-resolution spectra in the southern hemisphere (Henry et al. 1996) provided the source for the discovery of many rapidlyrotating solar-type stars (Soderblom et al. 1998). The H\&K work of W. Bidelman (e.g. Bidelman 1981; see also Sect. 2) supplied the target lists for the radial-velocity and photometric survey at SAAO (e.g. Balona 1987; Lloyd-Evans \& Koen 1987).

Another particularly important example of an usolved question in the above context is the angular momentum loss during stellar evolution. Magnetic braking of stellar rotation due to a stellar wind along predominantly equatorial magnetic field lines, like in our Sun, seemed not to have always the power to 
slow down stars from their initial angular momentum gained during the contraction from the pre-stellar cloud. The many ultra-fast rotators in young open clusters as well as the young field stars AB Dor, LQ Hya, EK Dra etc. are the most cited examples. Moreover, there exists a group of single, rapidlyrotating and evolved stars with strong magnetic activity (Fekel \& Balachandran 1993). This is a paradox since magnetic braking had enough time during the main-sequence stage to halt the rapid rotation, and the radius increase due to the termination of hydrogen-core burning should have resulted in an effectively complete loss of angular momentum. What process maintained these stars angular momentum? Is it the same process suggested for the ultra-rapid cluster rotators, i.e. a saturation of the atmospheric (coronal) volume with magnetic fields so that there is no torque arm for magnetic braking via a stellar wind anymore? Or is it something completely different?

Solanki et al. (1997), Strassmeier et al. (1998) and Buzasi (1999) suggested that magnetic fields concentrated in polar starspots could be the reason for such a lack of angularmomentum loss as described above. Solanki et al. presented numerical simulations that show that the effect would be quantitatively the same as with a dynamo saturation process. The only way to find conclusive observational evidence for or against the polar-spot hypothesis is to Doppler image these stars and search for polar starspots. Since Doppler imaging is an elaborate technique with many restrictions for the stellar sample (rapid rotation, medium inclination, known rotation period, relatively bright star etc.) one needs significantly more stellar candidates as known to date to cover the part in the $\mathrm{H}-\mathrm{R}$ diagram where stellar activity occurs. It is the primary aim of this survey to provide a larger sample of suitable Doppler-imaging targets. Additional goals are to provide activity-related stellar parameters like absolute Ca II emission-line fluxes, the $\mathrm{H} \alpha$ morphology, the lithium abundance, and photometric variations and relate them to absolute stellar parameters based on the distance from the Hipparcos satellite.

\section{Goals and observing procedure}

We have selected a subsample of late-type stars from the ESA Hipparcos catalog (ESA 1997) in the brightness range $7^{\mathrm{m}} 0-9{ }^{\mathrm{m}} 5$ and declination $-30^{\circ}$ through $+70^{\circ} . B-V$ colors between 0.67 and 1.0 for stars with parallaxes $\pi>20$ milli-arcsec (i.e. G5-K3 dwarfs) and between 0.87 and 1.2 for $3<\pi<20$ milli-arcsec (i.e. G5-K2 giants and subgiants) select the range of stars with convective envelopes and thus likely magnetic activity. Out of this sample of 6440 stars, 460 were observed in September 1998 in the right-ascension range $18^{\mathrm{h}}-6^{\mathrm{h}}$, and 598 in February 1999 in the right-ascension range $6^{\mathrm{h}}-18^{\mathrm{h}}$. Generelly, we excluded stars that already had a published moderate-to-high resolution Ca II spectrum but in some interesting cases we reobserved them. Previously known H\&K emission-line stars came mostly from the following sources: the original Wilson sample of photographic-plate spectra (e.g. Wilson 1976), the spectrophotometric Mt. Wilson H\&K survey (e.g. Duncan et al. 1991), Bidelman's catalogue and bibliography of emission-line stars of types later than B (Bidelman 1954), the Michigan-University southern and northern sky objective-prism survey (Bidelman \& MacConnell 1973; Bidelman 1981, 1983, 1985, 1988), the lists of Fekel and collaborators (e.g. Fekel et al. 1986), the CDS data base collected by Lastennet \& Freire Ferrero (1994), and our own Ca II data of active stars (Strassmeier et al. 1990, 1993; Strassmeier 1994).

Our observing procedure was as follows. After H\&K emission was detected from a short-exposure spectrum centered near $4000 \AA$, we reobserved the star at red wavelengths. These spectra include the $\mathrm{H} \alpha$ line and the lithium line at $6707 \AA$ and are used to determine their respective line properties and a more precise value for the rotational broadening as is possible from the blue spectra. The additional radial velocity from the red spectrum may also indicate whether the star is an unknown spectroscopic binary. It is also of higher precision due to lesser line blending in the red. If the star had H\&K emission, we added it to the observing menu of one of our two automatic photoelectric telescopes (APTs) in southern Arizona. These data are used to search for light variations and to determine a photometric period that is then assumed to be the stellar rotation period.

Figure 1 shows the sky coverage and galactic distribution of all target stars observed. Large dots denote the stars that were found to exhibit H\&K emission, small dots those without emission. Figure 2 plots the distribution of stellar parameters within the entire sample. $V$ magnitudes and $B-V$ colors were taken from the Tycho catalog and trigonometric parallaxes from the Hipparcos catalog (ESA 1997). The effective temperatures are based on the $B-V$ calibration from Flower (1996). Table A1 lists the stars with H\&K emission, Table A2 those without emission. Both tables are available only in electronic form.

\section{Observations}

\subsection{Spectroscopy}

All spectroscopic observations in this paper were obtained with the 0.9-m coudé feed telescope at Kitt Peak National Observatory (KPNO) during runs in September 10-23, 1998 and February 11 through March 3, 1999 (a few spectra were added from an earlier run in April 1998). Data were obtained with a $3000 \times 1000 \mathrm{CCD}$ (Ford F3KB chip, $15 \mu$ pixels) with grating $\mathrm{A}$, camera 5, the blue corrector, and the long collimator. Spectra were obtained at blue wavelengths centered at $4020 \AA$ to cover the two Ca II H\&K resonance lines at $3933 \AA$ and $3968 \AA$ as well as the luminosity-sensitive strontium line, Sr II, at $4077 \AA$. Stars with Ca II emission were also observed at red wavelengths centered at $6630 \AA$ to cover the Balmer $\mathrm{H} \alpha$ line at $6563 \AA$ and the neutral lithium line at $6708 \AA$. The useful wavelength coverage was $210 \AA$ in the blue region and $300 \AA$ in the red wavelength region. The resolving 


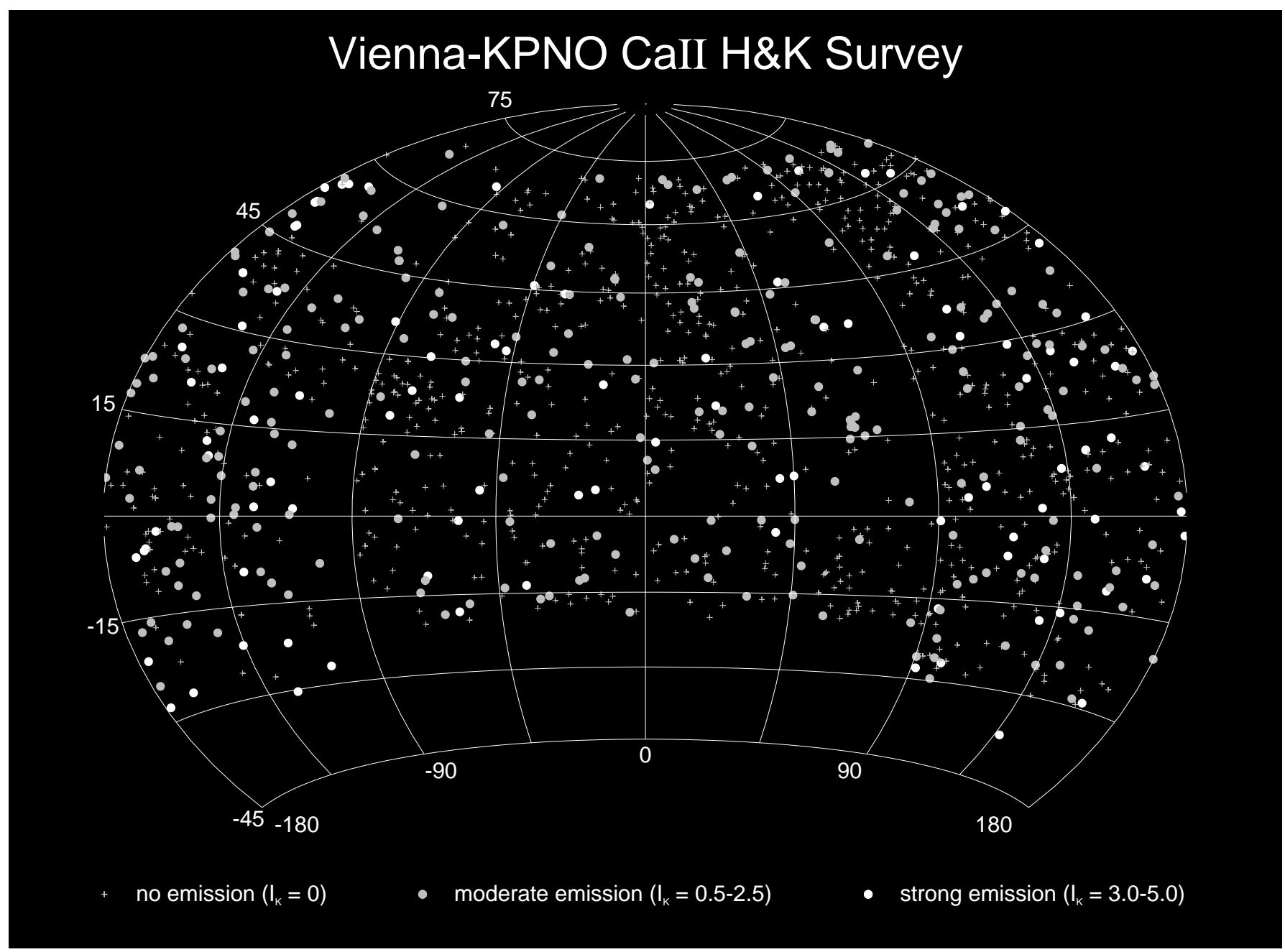

Fig. 1. The sky distribution of the sample of stars in this paper. Stars that were found to exhibit Ca II emission are shown as dots, non-emission stars as plusses. The active stars are again subdivided into weak-to-moderate emission (emission intensity, $I_{\mathrm{K}}=0.5-2.5$ according to Wilson (1976) and moderate-to-strong $\left(I_{\mathrm{K}}=3.0-5.0\right)$ emission stars

power, $\lambda / \Delta \lambda$, as measured from the full width at half maximum (FWHM) of the thorium-argon comparison lamp lines, was 18000 at $4020 \AA$ and 25000 at $6630 \AA$, This is an effective wavelength resolution of $0.23 \AA$ in the blue and $0.26 \AA$ in the red (at dispersions of $4.7 \AA / \mathrm{mm}$ and $7 \AA / \mathrm{mm}$, respectively). The instrumental FWHM was thereby sampled by 3.2 pixels and 2.5 pixels according to slit widths of $400 \mu \mathrm{m}$ in the blue and $280 \mu \mathrm{m}$ in the red, respectively. Unless otherwise noted, all blue spectra were obtained with an integration time of $5 \mathrm{~min}$ for stars brighter than $V=8$. 0 , with 7 min for stars between $8.0-8.8$, and with $10 \mathrm{~min}$ for stars fainter than 8.8 . This allows for a signal-to-noise $(\mathrm{S} / \mathrm{N})$ ratio in the continuum of approximately $20-40: 1$. The red-wavelength spectra were obtained with integration times between 20 and 30 minutes according to $\mathrm{S} / \mathrm{N}$ ratios between $70-150: 1$. A typical spectrum for each wavelength region is shown in Fig. 3.

All spectroscopic data were reduced with IRAF and included bias subtraction, flat fielding and optimized aperture extraction. Several wavelength comparison spectra and spectra of bright radial-velocity standards were obtained during each night to ensure an accurate wavelength calibration. Twenty flatfield exposures with a tungsten reference lamp were taken at the beginning of the night and again at the end of the night. These fourty flat fields were co-added and used to remove the pixelto-pixel variations in the stellar spectra on a nightly basis. The F3KB CCD showed no obvious signs of fringing at red wavelengths (none is expected in the blue) and no attempts were made to correct for it other than the standard flat-field division. Continuum fitting with a low-order polynomial was sufficient to find a satisfactory continuum solution. The H\&K region in the blue-wavelength spectra was excluded from the continuum solution.

\subsection{Photometry}

Follow-up photometry of most of the stars with Ca II H\&K emission was obtained with Wolfgang, one of the two $0.75-\mathrm{m}$ Vienna Observatory automatic photoelectric telescopes (APTs) 

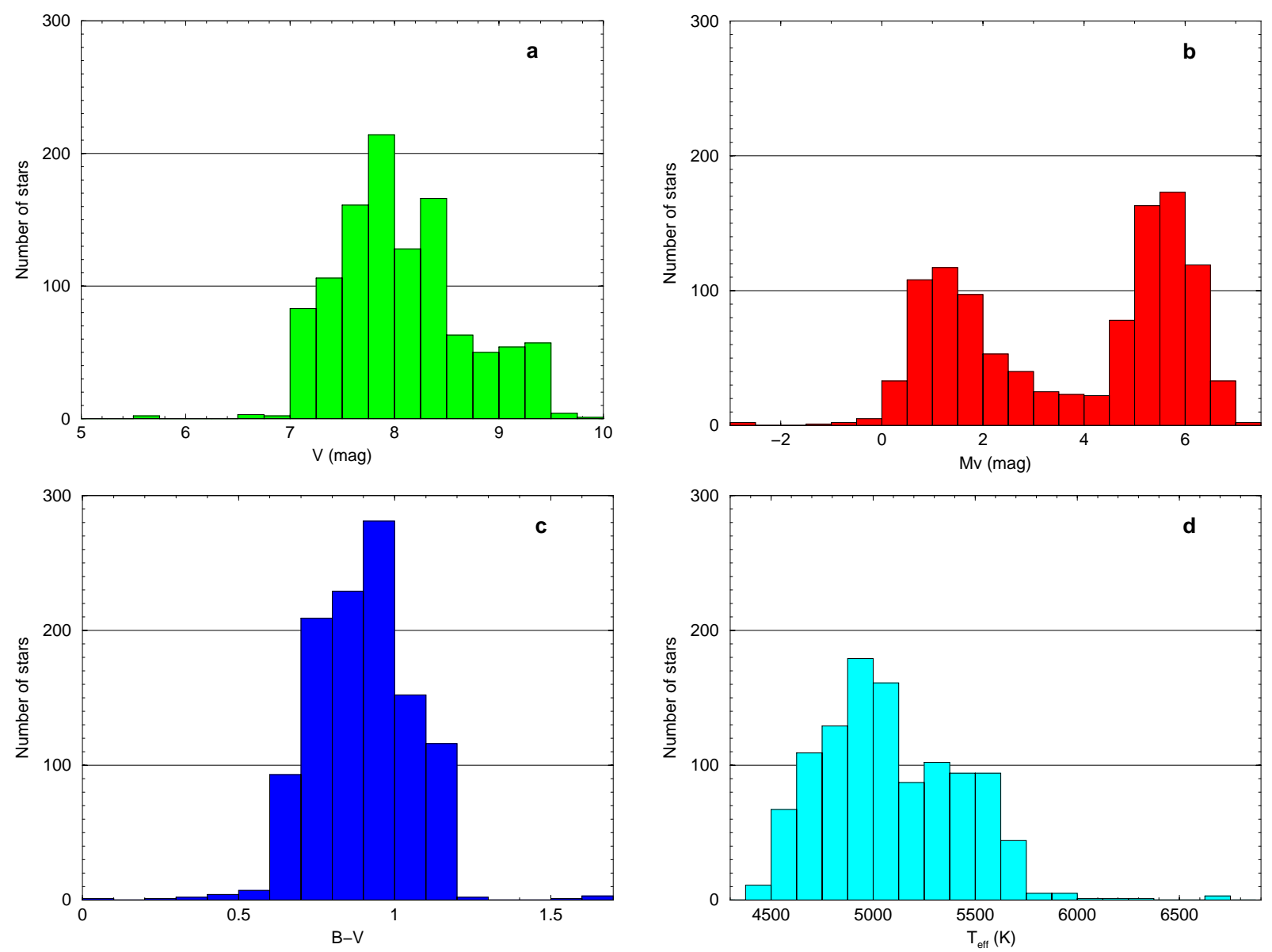

Fig. 2. Stellar parameters of the input sample. The four panels show the number of stars observed as a function of, a) visual brightness $V$, b) absolute magnitude $M_{V}$ (based on the Hipparcos parallax), c) $B-V$ color and d) effective temperature $T_{\text {eff }}$

at Fairborn Observatory in Arizona (Strassmeier et al. 1997b). All data were taken in and transformed to Strömgren $y$. Integration time was set to $20 \mathrm{~s}$ for each reading, except for stars fainter than $\approx 9^{\mathrm{m}}$ where 30 s were used.

Altogether, 8038 differential data points of 204 program stars are presented in this paper where each is the mean of three readings of the variable and four readings of the comparison star. This amount of data is proportional to $\approx 670$ hours of telescope time with $\approx 446$ hours of actual on-target integration. The observing sequence per target group was Nav-C2-Sky-CV-C-V-C-V-C-Sky-C2 (V=Variable). A second comparison star (C2), furtherin called the check star, was observed twice per differential group. A reading on the sky was taken before and at the end of each $\mathrm{V}-\mathrm{C}$ group while a bright navigation star (Nav) was recorded with the CCD finder as the first reading for each target group (these readings are for centering and are not of photometric quality). The standard error of a nightly mean from the overall seasonal mean was 0.003 in $y$ (for more details see Strassmeier et al. 1999).

\section{Results}

\subsection{New $H \& K$ emission-line stars and absolute surface fluxes}

Absolute H\&K emission-line fluxes are determined with the method of Linsky et al. (1979). It includes the measurement of the relative flux in a 50- $\AA$ band, $f_{50}$, between 3925 and $3975 \AA$ and the relative flux in the $\mathrm{H}$ and $\mathrm{K}$ emission lines, $f_{\mathrm{H}}$ and $f_{\mathrm{K}}$, as defined by the $(\mathrm{H} \& \mathrm{~K})_{1 \mathrm{~V}}$ and $(\mathrm{H} \& \mathrm{~K})_{1 \mathrm{R}}$ points, respectively. All relative fluxes are obtained by integrating the appropriate bandpass between the unnormalized spectrum and zero intensity.

The absolute emission line fluxes, $\mathcal{F}$, are calculated by comparing the ratio of the relative H\&K-line flux and the relative 50 - $\AA$ flux with a linear extrapolation of the absolute flux from the $V-R$ color index relation given by Linsky et al. (1979) and Strassmeier et al. (1994) and based upon the absolute photometry of Willstrop (1964):

$$
\begin{aligned}
\log \mathcal{F}_{50} & =8.264-3.076(V-R)_{\mathrm{J}} \\
\mathcal{F}_{\mathrm{H}} & =\frac{f_{\mathrm{H}}}{f_{50}}\left(50 \mathcal{F}_{50}\right) \\
\mathcal{F}_{\mathrm{K}} & =\frac{f_{\mathrm{K}}}{f_{50}}\left(50 \mathcal{F}_{50}\right) .
\end{aligned}
$$



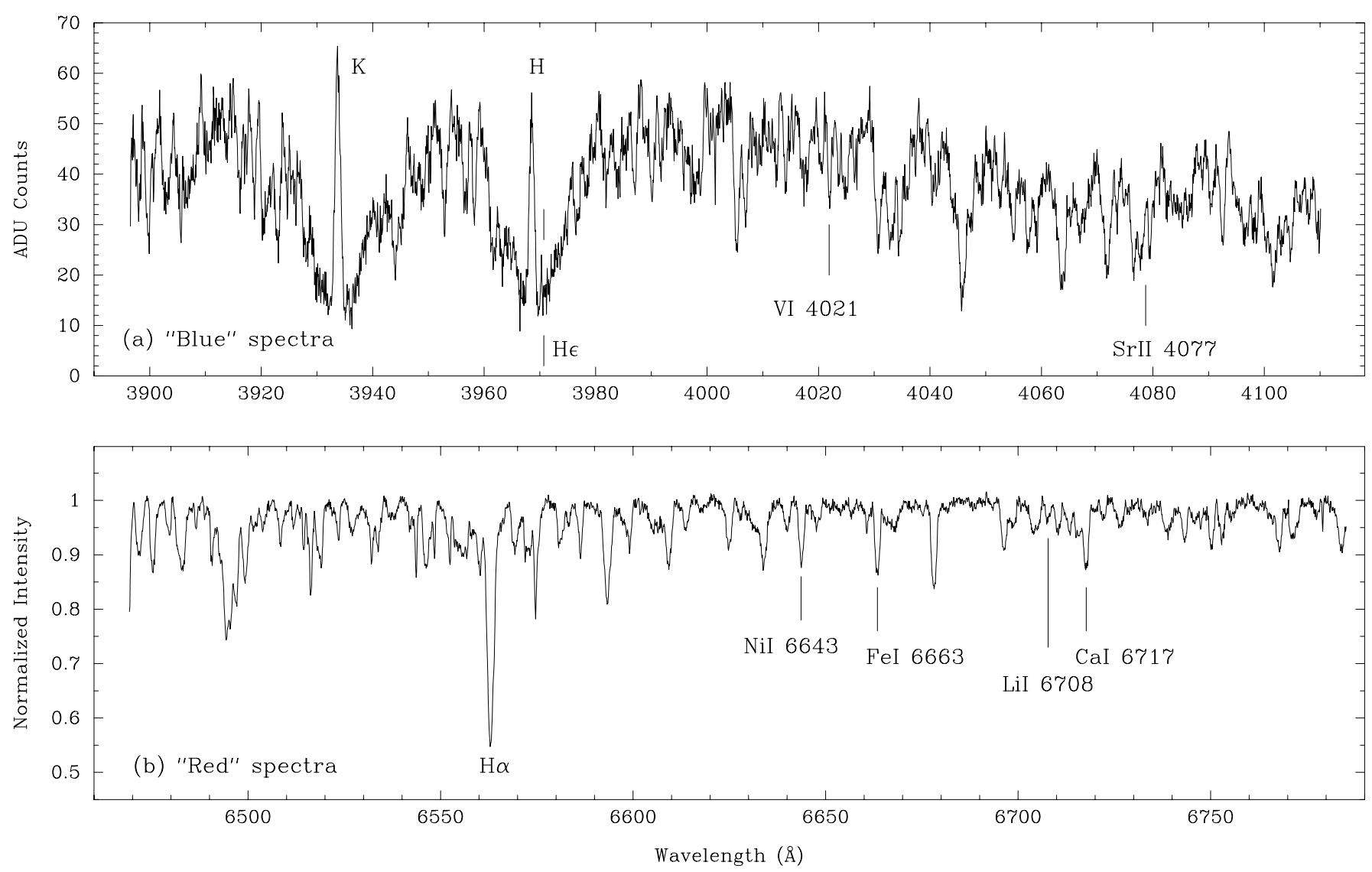

Fig. 3. Two examples of spectra obtained in this survey. Panel a) shows a blue-wavelength spectrum, panel b) a red-wavelength spectrum. Both spectra are for HD 553, a $V=8{ }^{\mathrm{m}} 1 \mathrm{~K} 0$ (sub)giant that was discovered to exhibit strong Ca II $\mathrm{H}$ and $\mathrm{K}$ emission. The spectral lines of interest are identified

Table A1 enumerates the results of this analysis and Table 1 is a quick-look summary of the stars with $\mathrm{H} \& \mathrm{~K}$ emission lines. In Table A1, Col. 1 lists the targets by HD number or, if unavailable, by Hipparcos number, as well as an eventual variable star name. Column 3 is the spectral classification taken from the Hipparcos-catalogue Appendix, and Col. 6 the trigonometric parallax from Hipparcos. Column 10 denotes the particular value of Johnson $(V-R)$ used in Eq. (1) to transform the relative flux ratio to the absolute flux. Note that the $V-R$ colors listed are not observed values but were computed from the observed Hipparcos $B-V$ color, the deduced absolute visual brightness, and the color-color relation tabulated in Gray (1992). Using $B-V$ instead of an observed $V-R$ value minimizes the effects of cool starspots and was shown by many authors (e.g. Strassmeier et al. 1994) to be a more consistent description of the unspotted photosphere. Columns 18 and 19 list the absolute H\&K emission-line fluxes and Cols. 20 and 21 the pure chromospheric fluxes. These fluxes were corrected for the photospheric contribution by subtracting the flux from a radiative equilibrium atmosphere, $\mathcal{F}_{\mathrm{H} \& \mathrm{~K}}^{\mathrm{RE}}$, given in Linsky et al. (1979) and represent the purely chromospheric emission flux, $\mathcal{F}_{\mathrm{H} \& \mathrm{~K}}^{\prime}$ :

$\mathcal{F}_{\mathrm{H} \& \mathrm{~K}}^{\prime}=\mathcal{F}_{\mathrm{H} \& \mathrm{~K}}^{\mathrm{obs}}-\mathcal{F}_{\mathrm{H} \& \mathrm{~K}}^{\mathrm{RE}}$.
These fluxes may be compared with the fluxes for the nonemission stars in Table A2 as well as with the basal fluxes from Rutten et al. (1991), which are believed to be due to acoustic heating of the chromosphere. The remaining flux difference for a given spectral type is then presumably of magnetic origin. For historical reasons, we add a column, $I_{\mathrm{K}}$, to Table A1 (Col. 11) that lists the Ca II K emission-line strengths according to Wilson's (1976) 0-5 scale; 0 denotes no detectable emission, and 5 marks strong emission lines reaching the nearby continuum or above. Figure B1 in the Appendix are Ca II plots of all stars with $\mathrm{H}$ and $\mathrm{K}$ emission stronger than $I_{\mathrm{K}} \geq 1$. Furthermore, we compute the sum of the corrected fluxes in the $\mathrm{H}$ and $\mathrm{K}$ line and express it in units of the bolometric luminosity (Col. 22):

$R_{\mathrm{HK}}=\frac{\mathcal{F}_{\mathrm{H}}^{\prime}+\mathcal{F}_{\mathrm{K}}^{\prime}}{\sigma T_{\mathrm{eff}}^{4}}$.

Fourteen stars had even $\mathrm{H} \epsilon$ in emission and we measured their absolute emission-line fluxes as well. These stars and their logarithmic $\mathrm{H} \epsilon$ emission-line fluxes in parenthesis in erg cm${ }^{-2} \mathrm{~s}^{-1}$ are: HD 553 (5.68), HIP 999 (6.57), HD 82286 (5.83), HD 95559 (6.03), HD 106855 (6.17), HD 127068 (5.92), HD 113816 (5.75), HIP 43422 (6.39), HIP 46634 (5.91), HIP 63322 (5.92), HD 145230 (5.85), HD 175742 (6.44), HD 178450 (6.52), and HD 218738 (6.43). The Ca II 
emission in the spectrum of HD 127068ab can not be unambiguously assigned to one of the two components. The two different entries for the emission strength of HD 141272 are real and indicate relatively large variations of the emission-line strength. The possible triple-lined system HD 139691 (=HIP 76563; see later in Sect. 4.5) has weaker emission than the usual inclusion limit for entries in Table A1 and we list it as a non-emission star in Table A2. Also note that its spectral-type entry in the Hipparcos/Tycho catalog reads F5 while the $B-V$ entry is $+1.7 \pm 0.5$. This indicates a composite spectrum.

Table A2 lists the results for the stars without detectable $\mathrm{H} \& \mathrm{~K}$ emission lines (Table 2 is a summary of their most commonly used identifications). Fluxes for these stars are determined from a $1-\AA$ band centered at the rest wavelengths of Ca II H\&K, respectively. Otherwise the procedure is similar as for the emission-line stars except the subtraction of the photospheric contribution. We expect an accuracy of the absolute chromospheric fluxes for these stars of the order of $30-50 \%$ based on a comparison with our previous, well-exposed KPNO spectra of $\gamma$ Dor candidates (Kaye \& Strassmeier 1998).

\subsection{Ho morphology and absolute fluxes}

The Balmer $\mathrm{H} \alpha$ line is an important indicator for chromospheric activity as well as for circumstellar emission and mass flow in late-type stars. Active stars have usually shallower $\mathrm{H} \alpha$ absorption than normal stars of similar spectral type and luminosity class while some of the very active stars of the RS CVn class even have $\mathrm{H} \alpha$ in emission. Our goal here is to verify the chromospheric nature of the $\mathrm{Ca}$ II $\mathrm{H}$ and $\mathrm{K}$ emission by an independent measure of the $\mathrm{H} \alpha$ core flux. We measure the inner $1-\AA$ portion of the $\mathrm{H} \alpha$ line from our continuum-normalized spectra and then relate it to the absolute continuum flux, $\mathcal{F}_{\mathrm{c}}$, at $\mathrm{H} \alpha$. The latter is obtained from the relations provided by Hall (1996) for various Morgan-Keenan (MK) classes:

$$
\begin{array}{r}
\mathcal{F}_{\mathrm{c}}=7.538-1.081(B-V) \\
\text { for MK I }-\mathrm{V} \text { and } 0<(B-V)<1.4 \\
\mathcal{F}_{\mathrm{c}}=7.518-1.236(V-R) \\
\text { for MK V and } 0<(V-R)<1.4 \\
\mathcal{F}_{\mathrm{c}}=7.576-1.447(V-R)
\end{array}
$$

for MK I - IV and $0<(V-R)<1.8$.

We compute the continuum flux from the calibrations in Eqs. (6) and (7) and use the average value for further processing. The $\mathrm{H} \alpha$-core flux in erg $\mathrm{cm}^{-2} \mathrm{~s}^{-1}$ (listed in Table A1 in Col. 24) is then computed from the measured 1- $\AA$ equivalent width under the spectrum, $W_{\text {core }}$ (Col. 23 in Table A1), and zero intensity:

$\mathcal{F}_{\mathrm{H} \alpha}=W_{\text {core }} \mathcal{F}_{\mathrm{c}}$.

Internal errors are estimated from repeated measurements of spectra taken during one night as well as from spectra of the same star obtained in different nights, and amount to no more than a few percent. External errors are mainly due to uncertainties in the absolute continuum flux due to errors in the colors and are estimated to be around $30 \%$. In case the star is a double-lined spectroscopic binary, we just give the combined flux unless two values appear as separate entries in Table A1.

Out of the total of $371 \mathrm{H} \& \mathrm{~K}$ emission-line stars, 46 have $\mathrm{H} \alpha$ in emission or significantly filled-in by emission. The panels in Fig. B2 in the Appendix contain plots of these stars around the $\mathrm{H} \alpha$-line region along with all other stars that have strong Ca II emission with $I_{\mathrm{K}} \geq 4$.

\subsection{Lithium detections and abundances}

The presence of a strong lithium line is generally a sign of stellar youth and thus indirectly also of stellar activity despite that it is still not fully clear how the lithium equivalent width relates to magnetic activity (e.g. Soderblom et al. 1993). More observations of lithium in active stellar atmospheres, especially in evolved stars that had enough time to deplete their primordial lithium, are needed to understand a possible link. In this paper, we present lithium measurements of 385 stars with $\mathrm{H}$ and $\mathrm{K}$ emission. Figure B3 in the Appendix shows plots of all stars with $W_{\mathrm{Li}} \gtrsim 10 \mathrm{~m} \AA$. Two stars (HD 144872 and HIP 82042) had an extended cosmic-ray hit at $6707.7 \AA$ and could not be measured.

We either fit a double Gaussian or, in case the Li line is stronger than $W_{\mathrm{Li}} \approx 70 \mathrm{~m} \AA$, a single Gaussian to the Li line. In some cases we directly integrate the area under two suitably chosen continuum points. Both tasks are carried out with IRAF's splot routine and result in typical internal errors of $3-5 \%$. However, if the Li-line strength is below $\approx 15 \mathrm{~m} \AA$, this error increases to $10-20 \%$ depending on the $\mathrm{S} / \mathrm{N}$ ratio of the spectrum. Note, that the equivalent widths in Table A1 include both lithium isotopes, i.e. ${ }^{6} \mathrm{Li}$ at $6707.76 \AA$ and ${ }^{7} \mathrm{Li}$ at $6707.91 \AA$ but exclude the nearby Fe I $6707.443+\mathrm{CN}$ blend unless otherwise noted. This is achieved by either a doubleGaussian fit or by fitting a symmetric profile to the red side of the lithium line. We estimate the lower limit for a positive detection of lithium to approximately $2-3 \mathrm{~m} \AA$ but it strongly depends on the $\mathrm{S} / \mathrm{N}$ ratio of the spectrum. Because blending with the nearby $\mathrm{Fe}$ I line is a major source of uncertainty for measuring small lithium equivalent widths, we estimate that our values in Table A1 have external uncertainties of $5-10 \%$ for $W_{\mathrm{Li}} \gtrsim 15 \mathrm{~m} \AA$ and $10-20 \%$ for $W_{\mathrm{Li}} \lesssim 15 \mathrm{~m} \AA$.

Lithium abundances are determined with the nonLTE curves of growth from Pavlenko \& Magazzù (1996). Respective effective temperatures are adopted from the Hipparcos $B-V$ color, listed again in Table A1 for reasons of completeness, and the calibration from Flower (1996). For effective temperatures between the values for which abundances are listed in Table 1 in Pavlenko \& Magazzù, we interpolate by fitting a third-order polynomial to the various entries. These abundances are uncertain by only 0.05 dex to $0.1 \mathrm{dex}$ when we propogate the uncertainties from the equivalent-width measurement. If we further assume an error of $\pm 100 \mathrm{~K}$ for the effective stellar temperatures, the uncertainties of our abundances increase to $0.15-0.16$ dex.

Throughout this paper, we give logarithmic abundances on a scale with $\log n(\mathrm{H})=12.00$. On this scale the observed 
Table 1. Stars with H\&K emission (data are listed in Table A1)

\begin{tabular}{|c|c|c|c|c|c|c|c|c|c|}
\hline HD 553 & HD 691 & HD 745 & HD 3125A & HD 4635 & HD 5835 & HD 5996 & HD 6665 & HD 6963 & HD 7205 \\
\hline HD 7286 & HD 7661 & HD 7895 & HD 8583 & HD 8997a & HD 8997b & HD 9313 & HD 9902b & HD 9902a & HD 10008 \\
\hline HD 12786 & HD 13357B & HD 13382 & HD 13507 & HD 13531 & HD 13579A & HD 14274 & HD 14374 & HD 15013 & HD 16287 \\
\hline HD 16884 & HD 17379 & HD 17382 & HD 18131 & HD 18632 & HD 18645 & HD $18955 b$ & HD 18955a & HD 19668 & HD 19902 \\
\hline HD 19942 & HD 20678 & HD 21663 & HD 21845 & HD 23140 & HD 23356 & HD 23386 & HD 23551 & HD 24053 & HD 25665 \\
\hline HD 25893 & HD 25998 & HD 26756 & HD 26900 & HD 27130a & HD 27130b & HD 27130 & HD 27149a & HD 27149b & HD 27282 \\
\hline HD 27466 & HD 27685 & HD 27989 & HD 28495 & HD 29883 & HD 30738 & HD 31000 & HD 35112 & HD 37216 & HD 40647 \\
\hline HD 40891 & HD 41067 & HD 41842 & HD 43516 & HD 43989 & HD 44573 & HD 45609 & HD 46183 & HD 46524 & HD 47787 \\
\hline HD 50255b & HD 50255a & HD 51866 & HD 52456 & HD 53157 & HD 53532 & HD 53927 & HD 54359 & HD 54371 & HD 56168 \\
\hline HD 59747 & HD 60491 & HD 61606 & HD 61994a & HD 61994b & HD 62668 & HD 64725 & HD 64942 & HD 65523 & HD 66553 \\
\hline HD 69247 & HD 69328 & HD 69433 & HD 70088 & HD 70146 & HD 71071 & HD 71251 & HD 71974 & HD 72146 & HD 72760 \\
\hline HD 73322 & HD 74150 & HD 75935 & HD 76218 & HD 76799 & HD 77825 & HD 78233 & HD 78644 & HD 79969 & HD 79993 \\
\hline HD 80355 & HD 81040 & HD 81659 & HD 81767 & HD 82159 & HD 82286a & HD $82286 b$ & HD 82443 & HD 82841 & HD 82939 \\
\hline HD 82977 & HD 83588 & HD 83983 & HD 85301 & HD 86065 & HD 86590a & HD 86590b & HD 86590c & HD 87424 & HD 87547 \\
\hline HD 87598 & HD 87883 & HD 87978 & HD 88638 & HD 88654 & HD 89546 & HD 89965 & HD 90442 & HD 91901 & HD 92945 \\
\hline HD 93811 & HD 93915 & HD 93915a & HD 93915b & HD 94765 & HD 95188 & HD 95559a & HD 95559b & HD $95559 \mathrm{c}$ & HD 95559 \\
\hline HD 95724 & HD 95743 & HD 96064 & HD 96612 & HD 97305 & HD 97601 & HD 98356 & HD 99303 & HD 100310 & HD 101206 \\
\hline HD 101906 & HD 102121 & HD 102195 & HD 102696 & HD 103072 & HD 103720 & HD 103847 & HD 104067 & HD 104243 & HD 104923 \\
\hline HD 105575a & HD $105575 b$ & HD $105575 c$ & HD 105631 & HD 105963A & HD 105963B & HD 106023 & HD 106156 & HD 106453 & HD 106711 \\
\hline HD 106855a & HD $106855 b$ & HD 108186 & HD 108564 & HD 108574 & HD 108575 & HD 108984 & HD 109011a & HD $109011 b$ & HD 109157 \\
\hline HD 109647 & HD 109703 & HD 110463 & HD 110514 & HD 110833 & HD 111312 & HD 111487 & HD 111487a & HD 111487b & HD 111813 \\
\hline HD 112099 & HD 112733 & HD 112859a & HD $112859 b$ & HD 113247 & HD 113247a & HD 113247b & HD 113449 & HD 113720 & HD 113816 \\
\hline HD 116544 & HD 116956 & HD 117099 & HD 117860 & HD 118234 & HD 119332 & HD 119607 & HD 120205 & HD 120352 & HD 121629 \\
\hline HD 121812 & HD 121979 & HD 122968 & HD 123351 & HD 124106 & HD 125874 & HD 126535 & HD 127068a & HD 127068b & HD 127871 \\
\hline HD 128165 & HD 128311 & HD 130004 & HD 130215 & HD 130307 & HD 130322 & HD 131023 & HD 131977 & HD 132425 & HD 134353 \\
\hline HD 136378 & HD 136834 & HD 137778 & HD 138134 & HD 138157 & HD 139194 & HD 139837 & HD 140637 & HD 141071 & HD 141272 \\
\hline HD 141919 & HD 142072 & HD 142680 & HD 143937a & HD 143937b & HD 144087 & HD 144088 & HD 144872 & HD 145230 & HD 147776 \\
\hline HD 147866 & HD 149028 & HD 149806 & HD 150202 & HD 150511 & HD 150748 & HD 152178 & HD 153525 & HD 153557 & HD 155712 \\
\hline HD $155802 \mathrm{a}$ & HD $155802 b$ & HD 158972 & HD 161284 & HD 163621 & HD 167715 & HD 168603 & HD 171488 & HD 172393 & HD 173950 \\
\hline HD 175742 & HD 176157 & HD 178450 & HD 180161 & HD 180263 & HD 180809 & HD 181219 & HD 183063 & HD 183870 & HD 186803 \\
\hline HD 189087 & HD 189733 & HD 190470 & HD 190642 & HD 192263 & HD 193479 & HD 196795 & HD 197913Aa & HD $197913 \mathrm{Ab}$ & HD $197913 B$ \\
\hline HD 198425 & HD 199967A & HD 199967B & HD 200560 & HD $200968 \mathrm{AB}$ & HD 201219 & HD 202605 & HD 203030 & HD 203136 & HD 205249 \\
\hline HD 205762 & HD 206374 & HD 207485 & HD 207583 & HD 208313 & HD 208472 & HD 209154 & HD 209393 & HD 209779 & HD 210667 \\
\hline HD 211472 & HD $214615 \mathrm{AB}$ & HD 214683 & HD 215274 & HD 215555 & HD 217352 & HD 217580 & HD 218153 & HD 218738 & HD 218739 \\
\hline HD 220182 & HD 220476 & HD 221851 & HD 222422 & HD 223154 & HD 223941 & HD 224983 & HD 237944a & HD 237944b & HD $237944 c$ \\
\hline HD 258857 & HD 261557 & HD 263175 & HD 285931 & HD 291095 & BH Vir a & BH Vir b & CG Cyg a & CG Cyg b & HIP 999 \\
\hline HIP 6339 & HIP 36357 & HIP 39222 & HIP 40774 & HIP 42253 & HIP 43418 & HIP 43422 & HIP 43751 & HIP 46634 & HIP 47176 \\
\hline HIP 50072 & HIP 50660 & HIP 51197 & HIP 56299 & HIP 57859 & HIP 58560 & HIP 59152 & HIP 59904 & HIP 63322a & HIP 63322b \\
\hline HIP 63442 & HIP 64059 & HIP 69410 & HIP 70836 & HIP 75011 & HIP 77179 & HIP 77210a & HIP $77210 b$ & HIP 78688 & HIP 82042 \\
\hline HIP 83141 & HIP 101227 & SAO $150676 a$ & SAO $150676 b$ & SAO $151224 a$ & SAO $151224 b$ & & & & \\
\hline
\end{tabular}

solar photospheric Li abundance listed by Grevesse \& Anders (1991) is $1.16 \pm 0.1$, and the Li-6708 line appears to have an equivalent width of around $2 \mathrm{~m} \AA$. This value is comparable to the detection limit from our spectra. If an entry in Table A1 is zero, then no Li above $\approx 2-3 \mathrm{~m} \AA$ was detected. An entry with $\lesssim 3 \mathrm{~m} \AA$ means a very weak lithium line is likely present but is unreliably small to be measured. The largest equivalent widths in our sample were measured for HD 140637 (420 mÅ),

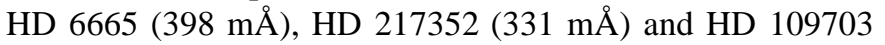
(300 m $\AA$ ), which basically amount to the primordial lithium abundance. Out of the 385 stars in our (red) sample, $102(26 \%)$ had undetectable Li, 119 (31\%) had Li below $10 \mathrm{~m} \AA, 128$ (33\%) between $10-99 \mathrm{~m} \AA$, and 36 (9.3\%) more than $100 \mathrm{~m} \AA$ (detections in SB2s and SB3s are counted only once). Two stars in Table A2 that have no H\&K emission but were we obtained a red-wavelength spectrum seem to have significant lithium: HD 32915 with $52 \mathrm{~m} \AA(\log n(\mathrm{Li})=1.57)$ and HD 123999 with $18 \mathrm{~m} \AA(\log n(\mathrm{Li})=1.22)$. Both stars appear to be single.

\subsection{Strontium $\mathrm{Sr}$ II $4077-\AA$ line strength}

Sr II 4077 was noted to be a primary luminosity indicator for late F, G, and even K and M stars (Gray \& Garrison 1989). The solar spectrum shows Sr II 4077 as a triplet line (4077.580,
$4077.724,4077.834$ ) with a total equivalent width of $428 \mathrm{~m} \AA$. However, the line is by far dominated by the 4077.724- $\AA$ transition with a low excitation potential of zero.

We fit a Gaussian or, if inappropriate due to saturation, a Voigt function to the Sr II blend and measure its equivalent width and residual intensity. Our fitting procedure minimizes the influences of the nearby La II and Cr II+Ce II blends on the blue side of the line by fitting mostly the red wing of the Sr II profile. Repeated measurements show an internal error of the equivalent width of less than $10 \%$ but continuum uncertainties, rotational broadening, low signal-to-noise ratio, and heavy blending account for an estimated external error of $\approx 20-40 \%$. The residual intensity, i.e. line depth measured from the continuum, is taken from the minimum of the Gaussian fit. The numerical results are listed in Table A1 and Table A2.

\subsection{Radial velocities and binarity}

Radial velocities were derived from the blue-wavelength spectra and the red-wavelength spectra by cross-correlating them with spectra of velocity standard stars taken during the same night. The following velocity standards were adopted from Scarfe (1990): $\beta$ Oph (K2III, $v_{\mathrm{r}}=-12.18 \mathrm{~km} \mathrm{~s}^{-1}$ ) 
Table 2. Stars without H\&K emission (data are listed in Table A2)

\begin{tabular}{|c|c|c|c|c|c|c|c|c|c|}
\hline HD 15 & HD 443 & HD 471 & HD 533 & HD 629 & HD 1036 & HD 1059 & HD 1153 & HD 1241 & HD 1350 \\
\hline HD 1426 & HD 1449 & HD 1605 & HD 1624 & HD 2034 & HD 2622 & HD 2712 & HD 2805 & HD 2814 & HD 2816 \\
\hline HD 2841 & HD 3141 & HD 3250 & HD 3400 & HD 3681 & HD 3765 & HD 4029 & HD 4372 & HD 4388 & HD 4449 \\
\hline HD 4561 & HD 4744 & HD 4754 & HD 4770 & HD 5035 & HD 5279 & HD 5349 & HD 5747 & HD 5873 & HD 5897 \\
\hline HD 6360 & HD 6448 & HD 6555 & HD 6645 & HD 6890 & HD 7018 & HD 7149 & HD 7234 & HD 7385 & HD 7444 \\
\hline HD 7639A & HD 7864 & HD 7980 & HD 8016 & HD 8129 & HD 8275 & HD 8389 & HD 8508 & HD 8553 & HD 8561 \\
\hline HD 8594 & HD 8654 & HD 8828 & HD 8910 & HD 9070 & HD 9304 & HD 9342 & HD 9556 & HD 9847 & HD 9938 \\
\hline HD 10126 & HD 10145 & HD 10196 & HD 10304 & HD 10311 & HD 10743 & HD 10755 & HD 11286 & HD 11443 & HD 11707 \\
\hline HD 11979 & HD 12051 & HD 12343 & HD 12661 & HD 13357A & HD 13783 & HD 13997 & HD 14204 & HD 14648 & HD 14787 \\
\hline HD 14798 & HD 14855 & HD 14940 & HD 15014 & HD 15015 & HD 15096 & HD 15116 & HD 15299 & HD 15682 & HD 15734 \\
\hline HD 15830 & HD 15851 & HD 16293 & HD 16366 & HD 16454 & HD 16559 & HD 16674 & HD 17190 & HD 17655 & HD 17673 \\
\hline HD 18143 & HD 18175 & HD 18200 & HD 18916 & HD 19308 & HD 20165 & HD 20215 & HD 21774 & HD 21864 & HD 22233 \\
\hline HD 22657 & HD 22854 & HD 23065 & HD 23439A & HD 23439B & HD 24206 & HD 24238 & HD 24316 & HD 24467 & HD 24485 \\
\hline HD 24604 & HD 24782 & HD 24964 & HD 25242 & HD 25347 & HD 26004 & HD 26334 & HD 26397 & HD 26465 & HD 26633 \\
\hline HD 26634 & HD 27040 & HD 27115 & HD 27126 & HD 27235 & HD 27275 & HD 27574 & HD 28115 & HD 28172 & HD 28185 \\
\hline HD 28338 & HD 28488 & HD 28821 & HD 30385 & HD 31439 & HD 31452 & HD 31865 & HD 31867 & HD 32237 & HD 32547 \\
\hline HD 32915 & HD 32935 & HD 33142 & HD 33725 & HD 34101 & HD 34153 & HD 34616 & HD 34623 & HD 34839 & HD 34909 \\
\hline HD 35518 & HD 37008 & HD 37986 & HD 38313 & HD 38904 & HD 39169 & HD 39847 & HD 39855 & HD 40708 & HD 41017 \\
\hline HD 41196 & HD 41241 & HD 41303 & HD 41483 & HD 41785 & HD 41788 & HD 42182 & HD 42250 & HD 42532 & HD 42568 \\
\hline HD 42606 & HD 42891 & HD 42983 & HD 43050 & HD 43062 & HD 43147 & HD 43188 & HD 43259 & HD 43306 & HD 43710 \\
\hline HD 43944 & HD 44199 & HD 44263 & HD 44420 & HD 44671 & HD 45140 & HD 45231 & HD 45350 & HD 45652 & HD 45762a \\
\hline $\mathrm{HD} 45762 \mathrm{~b}$ & HD 46090 & HD 46093 & HD 46375 & HD 46691 & HD 47157 & HD 47185 & HD 47186 & HD 47309 & HD 47955 \\
\hline HD 47963 & HD 48238 & HD 48878 & HD 49027 & HD 49039 & HD 49116 & HD 49165 & HD 49566 & HD 49649 & HD 49674 \\
\hline HD 50275 & HD 50590 & HD 50630 & HD 50662 & HD 51046 & HD 52145 & HD 54240 & HD 54401 & HD 55076 & HD 55255 \\
\hline HD 55458 & HD 55647 & HD 55672 & HD 55893 & HD 57204 & HD 57470 & HD 57473 & HD 57678 & HD 57729 & HD 57758 \\
\hline HD 57813 & HD 57901 & HD 58595 & HD 58662 & HD 58781 & HD 58899 & HD 59062 & HD 59560 & HD 60272 & HD 60299 \\
\hline HD 60368 & HD 61145 & HD 61381 & HD 62676 & HD 63536 & HD 64114 & HD 64143 & HD 64211 & HD 64468 & HD 64490 \\
\hline HD 64606 & HD 64921 & HD 65069 & HD 65371 & HD 65430 & HD 65562 & HD 65854 & HD 66046 & HD 66177 & HD 66221 \\
\hline HD 66485 & HD 66509 & HD 66983 & HD 67740 & HD 67850 & HD 67960 & HD 68586 & HD 68638 & HD 69056 & HD 69076 \\
\hline HD 69613 & HD 70352 & HD 71811 & HD 71887 & HD 72003 & HD 72614 & HD 72769 & HD 72946 & HD 73393 & HD 73512a \\
\hline HD 73512b & HD 73536 & HD 73667 & HD 74014 & HD 74377 & HD 75031 & HD 75073 & HD 75318 & HD 75697 & HD 75879 \\
\hline HD 76294 & HD 76632 & HD 76752 & HD 76780 & HD 76844 & HD 76849 & HD 76909 & HD 77278 & HD 77338 & HD 77711 \\
\hline HD 77712 & HD 79498 & HD 79581 & HD 79643 & HD 80131 & HD 80367 & HD 80448 & HD 81110 & HD 81438 & HD 81744 \\
\hline HD 82460 & HD 82733 & HD 83804 & & & HD 85987 & HD 86661 & & & HD 87836 \\
\hline HD 89023 & HD 89813 & HD 90107 & HD 90663 & HD 90711 & HD 90735 & HD 90812 & HD 90820 & HD 90932 & HD 91148 \\
\hline HD 91299 & HD 91453 & HD 91527 & HD 91585 & HD 92048 & HD 92213 & HD 92320 & HD 92786 & HD 92788 & HD 93650 \\
\hline HD 93800 & HD 94119 & HD 94164 & HD 94374 & HD 94718 & HD 94783 & HD 94880 & HD 95246 & HD 95848 & HD 96027 \\
\hline HD 96460 & HD 96937 & HD 97004 & HD 97343 & HD 97658 & HD 98055 & HD 98078 & HD 98186 & HD 98281 & HD 98736 \\
\hline HD 98839 & HD 98959 & HD 99994 & HD 100922 & HD 101227 & HD 101534 & HD 101728 & HD 102494 & HD 102800 & HD 103431 \\
\hline HD 103432 & HD 104163 & HD 104782 & HD 104906 & HD 104988 & HD 105844 & HD 106495 & HD 107469 & HD 108849 & HD 109402 \\
\hline HD 111285 & HD 111515 & HD 111978 & HD 112742 & HD 112758 & HD 112815 & HD 112914 & HD 114060 & HD 114125 & HD 114260 \\
\hline HD 114783 & HD 114784 & HD 114823 & HD 115080 & HD 115153 & HD 115638 & HD 115755 & HD 116012 & HD 116056 & HD 116093 \\
\hline HD 116442 & HD 116443 & HD 117635 & HD 118670 & HD 119585 & HD 119932 & HD 121129 & HD 121249 & HD 121320 & HD 122562 \\
\hline HD 122676 & HD 122948 & HD 123265 & HD 123399 & HD 124292 & HD 124677 & HD 125056 & HD 125455 & HD 125920 & HD 126511 \\
\hline HD 126532 & HD 126583 & HD 127352 & HD 1 & D 128356 & HD 128731 & HD 129674 & HD 130669 & HD 131509 & HD 132307 \\
\hline HD 132756 & HD 133352 & HD 134043 & HD 134439 & HD 134440 & HD 134985 & HD 135725 & HD 136136 & HD 136274 & HD 136655 \\
\hline HD $136655 b$ & HD 136894 & HD 138885 & HD 138919 & HD 139691a & HD $139691 \mathrm{~b}$ & HD $139691 \mathrm{c}$ & HD 142478 & HD 143990 & HD 144287 \\
\hline HD 144873 & HD 147512 & HD 149933 & D 150510 & HD 150665 & HD 151192 & HD 151504 & HD 151528 & HD 151541 & HD 151877 \\
\hline HD 152275 & HD 153402 & HD 154510 & HD 158332 & HD 159062 & HD 164809 & HD 164853 & HD 164922 & HD 164923 & HD 164986 \\
\hline HD 165168 & HD 165169 & HD 165173 & HD 165807 & HD 166498 & HD 166683 & HD 167081 & HD 167450 & HD 167858 & HD 168744 \\
\hline HD 168746 & HD 169797 & HD 169822 & HD 169889 & HD 170232 & HD 170738 & HD 171010 & HD 171067 & HD 171215 & HD 171920 \\
\hline HD 172043 & HD 172132 & HD 172245 & HD 172310 & HD 172586A & HD 173399 & HD 173872 & HD 174000 & HD 174719 & HD 175516 \\
\hline HD 175518 & HD 175905 & HD 176410 & HD 176646 & HD 176650 & HD 176733 & HD 177699 & HD 177778 & HD 178326 & HD 178473 \\
\hline HD 178541 & HD 178848 & HD 179558 & HD 179722 & HD 180642 & HD 181007 & HD 181047 & HD 181098 & HD 181421 & HD 182293 \\
\hline HD 182619 & HD 183098 & HD 183418 & HD 183993 & HD 184591 & HD 184592 & HD 184768 & HD 185055 & HD 185147 & HD 185353 \\
\hline HD 185413 & HD 1855 & HD 186196 & HD 187000 & HD 188168 & HD 188386 & HD 188522 & HD 189751 & HD 190067 & HD 190404 \\
\hline HD 190412 & HD 190536 & HD 190873 & HD 191425 & HD 191499A & HD 191785 & HD 192732 & HD 192773 & HD 193116 & HD 193953 \\
\hline HD 195220 & HD 195987 & HD 196689 & HD 196692 & HD 197210 & HD 197274 & HD 197396 & HD 197657 & HD 197737 & HD 198402 \\
\hline HD 198456 & HD 198482 & HD 199580 & HD 199660 & HD 200213 & HD 200386 & HD 201270 & HD 201702 & HD 201924 & HD 202109 \\
\hline HD 202365 & HD 202585 & HD 202620 & HD 202751 & HD 202835 & HD 202999 & HD 203384 & HD 203712 & HD 204079 & HD 204814 \\
\hline HD 205286 & HD 205321 & HD 205606 & HD 206243 & HD 206557 & HD 206928 & HD 206993 & HD 207032 & HD 207372 & HD 207487 \\
\hline HD 207740 & HD 207771 & HD 207839 & HD 207874 & HD 207966A & HD 208201 & HD 208398 & HD 208880 & HD 209181 & HD 209262 \\
\hline HD 209776 & HD 210123 & HD 210144 & HD 211513 & HD 211642 & HD 212094 & HD 212291 & HD 212587 & HD 212771 & HD 212989 \\
\hline HD 213012 & HD 213764 & HD 213786 & HD 213920 & HD 215097 & HD 215144 & HD 215152 & HD 215183 & HD 215500 & HD 215696 \\
\hline HD 215704 & HD 215775 & HD 215886 & HD 216103 & HD 216259 & HD 216284 & HD 216560 & HD 216572 & HD 217635 & HD 218105 \\
\hline HD 218220 & HD 218790 & HD 218949 & HD 219029 & HD 219202 & HD 219514 & HD 219670 & HD 219829 & HD 219920 & HD 220339 \\
\hline HD 220658 & HD 220871 & HD 221194 & & & & HD 222405 & HD 222455 & HD 223070 & HD 223301 \\
\hline HD 223302 & HD 223374 & HD 223498 & HD 223662 & HD 223847 & HD 223971 & HD 224116 & HD 224844 & HD 225004 & HD 225021 \\
\hline HD 225170 & HD 225242 & HD 225261 & HD 226099a & HD $226099 \mathrm{~b}$ & HD 232118 & HD 233373 & HD 233389 & HD 233608 & HD 233826 \\
\hline HD 233874 & HD 233882 & & & & & HD 237742 & HD 237960 & HD 238015 & HD 238130 \\
\hline HD 250047 & HD 251383 & HD 255639 & HD 257886 & HD 337457 & HD 347850 & HD 347898 & $31 \mathrm{Com}$ & HIP 4114 & HIP 8358 \\
\hline HIP 13338 & HIP 29814 & & HIP 34498 & HIP 34866 & HIP 35534 & HIP 35989 & HIP 36522 & HIP 37146 & HIP 39883 \\
\hline & & & & & & HIP 47975 & HIP 48786 & HIP 49104 & HIP 50638 \\
\hline HIP 52782 & HIP 56408 & HIP 56570 & HIP 59321 & HIP 62911 & HIP 64706 & HIP 65221 & HIP 65485 & HIP 65837 & HIP 66931 \\
\hline HIP 69142 & HIP 76112 & HIP 76566 & HIP 78068 & HIP 81831 & HIP 83630 & HIP 88208 & HIP 92881 & HIP 94075 & SAO 45472 \\
\hline
\end{tabular}



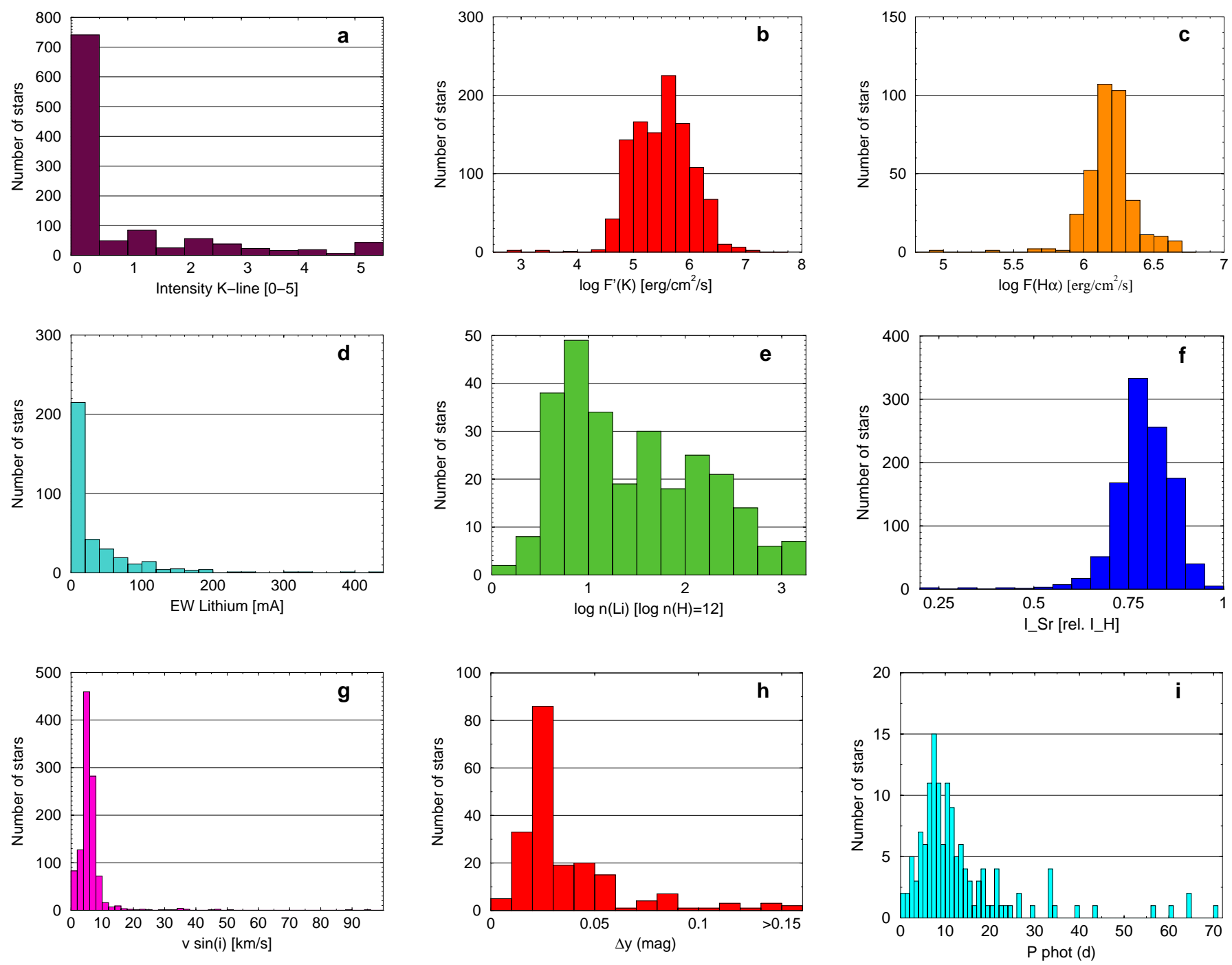

Fig. 4. Histograms for the survey results. a) Ca II K-line intensities $\left(I_{\mathrm{K}}\right)$. The scale is from 0 (no emission) to 5 (emission at or above the continuum) according to Wilson (1976). b) Absolute emission-line fluxes for the Ca II-K line $\left(\log \mathcal{F}^{\prime}(K)\right)$. c) Absolute emission-line fluxes for $\mathrm{H} \alpha(\log \mathcal{F}(\mathrm{H} \alpha))$. d) Observed Li I 6708- $\AA$ equivalent widths in milli- $\AA$ ( $\left.E W_{\mathrm{Li}}\right)$. e) Logarithmic lithium abundances, $\log n(\mathrm{Li})$, in units of the hydrogen abundance $(\log n(\mathrm{H})=12.00)$. f) Line-core intensity of the Sr II 4077- $\AA$ line $\left(I_{\text {SrII }}\right)$ with respect to the continuum. g) Rotational line broadening in $\mathrm{km} \mathrm{s}^{-1}(v \sin i)$. h) Photometric $y$ amplitudes in magnitudes $(\Delta y)$. i) Rotation periods in days $\left(P_{\text {rot }}\right)$

$\alpha$ Ari (K2IIIab, $\left.v_{\mathrm{r}}=-14.51 \mathrm{~km} \mathrm{~s}^{-1}\right), \beta$ Gem (K0III, $v_{\mathrm{r}}=$ $+3.23 \mathrm{~km} \mathrm{~s}^{-1}$ ), and $35 \mathrm{Peg}\left(\mathrm{K} 1 \mathrm{III}-\mathrm{IV}, v_{\mathrm{r}}=+54.26 \mathrm{~km} \mathrm{~s}^{-1}\right)$. At least two spectra of standard stars were obtained each night. All cross correlations were computed with IRAF's fxcor routine. It fits one or more Gaussians to the crosscorrelation function in case the star is a double or triple-lined spectroscopic binary, respectively. A brief description of the measuring procedure and several applications to spotted stars were presented and discussed by Fekel et al. (1999). Several of the cross-correlation functions in the present paper appear asymmetric due to the presence of cool starspots. Measuring the velocity from a fit to the peak of the cross-correlation function would result in a less accurate velocity. Therefore, our Gaussian fits were computed to fit the entire cross-correlation profile, and not just the peak. Differences between such fits can amount to up to $3 \mathrm{~km} \mathrm{~s}^{-1}$ for the most asymmetric cases. Errors due to spectral-type mismatch between reference stars and program stars depend upon rotational broadening and add to the total error. Several tests with different reference stars gave negligible internal errors for $v \sin i \lesssim 10 \mathrm{~km} \mathrm{~s}^{-1}$, approximately $\pm 0.1 \mathrm{~km} \mathrm{~s}^{-1}$ for $v \sin i$ up to $20 \mathrm{~km} \mathrm{~s}^{-1}$ and $\approx 0.3 \mathrm{~km} \mathrm{~s}^{-1}$ up to $50 \mathrm{~km} \mathrm{~s}^{-1}$. These errors are considered small and are not included in the quoted errors in this paper. Our results are listed in Tables A1 and A2 along with the errors $\left(\sigma_{\mathrm{vr}}\right)$ from the Gaussian fit. Note that the spectral region around the Ca II resonance lines $(3920-3980 \AA$ ) was excluded from the blue-wavelength cross correlations, as was the $\mathrm{H} \alpha$ region $(6558-6568 \AA)$ from the red-wavelength cross correlations.

Our sample contains altogether 36 single-lined spectroscopic binaries (SB1), 17 of them are new detections (according to Simbad). The newly discovered SB1s are HD 553, HIP 999, HD 16884, HD 62668, HD 78644, HIP 46634, HD 82159, HD 82841, HIP 50072, HD 112099, HIP 63322, 
HIP 63442, HD 138157, HD 142680, HD 150202, HD 153525 , and HD 190642. A further 16 targets were found to be possible SB1s. In these cases the difference in velocity from the red to the blue spectrum was still larger than the sum of their uncertainties plus $1 \sigma$. These targets are HD 18645, HD 24053, HD 23551, HD 40891, HD 43516, HD 66553, HD 76799, HD 95188, HD 95724, HD 104067, HD 105963B, HD 120205, HD 147866, HD 171067, HD 184591, and HD 218739. The latter forms a visual pair with HD 218738 (=KZ And), a well-known active binary and listed in the CABS catalog.

For the cases where the cross-correlation function was double peaked, we fitted a double Gaussian to it and list the individual velocities and their errors in separate rows in Table A1 and Table A2. The stellar component with the stronger absorption lines is always called the primary (indicated by the suffix "a"). The component with the weaker line is called component " $\mathrm{b}$ ". Altogether, 30 double-lined spectroscopic binaries (SB2) and two triple-lined spectroscopic binaries (SB3) are in our sample. The latter are HD 86590 (DH Leo), and HD 237944A. HD 237944A appears to be a newly discovered SB3 system. HD 95559, HD 139691 (ADS $9731 \mathrm{AB}$ ) and HIP 76563 appear triple lined in the crosscorrelation function from a single blue or red spectrum but that needs confirmation. At the moment, we list them as possible triple-lined systems and give three velocities in Tables A1 or A2. The double-lined systems are HD 8997, HD 9902, HD 18955, HD 27130, HD 27149, SAO 150676, SAO 151224, HD 45762, HD 50255, HD 61994, HD 73512, HIP 77210, HD 82286, HD 93915, HD 95559 (possible SB3), HD 105575, HD 106855, HD 109011, HD 111487, HD 112859, BH Vir, HD 127068, HD 136655, HD 143937, HD 155802, CG Cyg, HD 197913A, HD 199967AB, HD 202109, and HD 226099. Out of these 30 systems, 19 are new detections. The binary components of HD 199967AB are not resolved at the entrance slit and the radial velocity for component $\mathrm{B}$ from the blue spectrum is very uncertain due to a double-peaked crosscorrelation peak. We can not decide whether it is truly doubled or just spurious. In any case, the velocity from the stronger of the two peaks is listed in Table A1 (the weaker is at $31.0 \pm$ $5.9 \mathrm{~km} \mathrm{~s}^{-1}$ ). The spectrum of the RS CVn binary CG Cyg (CABS \# 177) appears to consist of two very broad lines at $-67 \pm 15 \mathrm{~km} \mathrm{~s}^{-1}$ and $+65 \pm 23 \mathrm{~km} \mathrm{~s}^{-1}$, respectively, and one very sharp system of lines at $-0.6 \pm 4.3 \mathrm{~km} \mathrm{~s}^{-1}$, practically at the binary's center-of-mass velocity. The latter line system may be due to a third star but we can not exclude an absorption spectrum due to circumbinary material as suggested by Milone $\&$ Naftilan (1980). At the moment, we continue to list the star as a SB2. A similar case is HD 105575 which is classified as a $\beta$ Lyrae-type eclipsing binary in Simbad. Our spectrum shows moderately strong and sharp H\&K emission lines while another system of broad absorption lines is present as well. We suggest that the sharp lines are from circumbinary material and the broad lines from one of the two stellar components. Note that its $\mathrm{H} \alpha$ line appears to be tripled and shows another cross-correlation peak at $130 \pm 4 \mathrm{~km} \mathrm{~s}^{-1}$.
Three systems are identified with radial velocities well above $100 \mathrm{~km} \mathrm{~s}^{-1}$. HD 108564, a single star with weak Ca II emission and a single velocity measure of $v_{r}=+111 \mathrm{~km} \mathrm{~s}^{-1}$, HD 142680, a single-lined spectroscopic binary with weak emission and two velocity measures of $v_{r}=-111$ and $-83 \mathrm{~km} \mathrm{~s}^{-1}$. It has a double-peaked cross-correlation function but the spectrum shows no clear evidence of the secondary lines. We list it as a SB1 but it could to be an unresolved SB2 system. If so, the second peak in the red spectrum gives $v_{\mathrm{r}}=-52.5 \pm 3.5 \mathrm{~km} \mathrm{~s}^{-1}$. HD 143937, a double-lined spectroscopic binary with strong Ca II emission and with peak velocities of even -166 and $-170 \mathrm{~km} \mathrm{~s}^{-1}$ for the primary and secondary, respectively. As a comparison, a recent discovery of an extreme runaway star (HIP 60350; Maitzen et al. 1998) with $+220 \mathrm{~km} \mathrm{~s}^{-1}$ pointed in the direction of a dynamical cluster ejection rather than to a supernovae scenario.

In case a close visual component was spatially resolved at the spectrograph entrance slit and two separate spectra were obtainable, we adopt the notation that the brighter of them is denoted component " $\mathrm{A}$ ", and the fainter component "B". The A-component of the close visual binary HD 197913 turned out to be a SB2 with components Aa and Ab.

\subsection{Space motions}

Space motions are computed with the fundamental Hipparcos data (positions, distances, and proper motions) and our radial velocities. For the few entries without a Hipparcos parallax the value listed in Simbad was adopted and, if no value was listed in Simbad, we used the assigned luminosity class and the visual magnitude to determine an approximate distance (this was done for three stars: HD 9902, HD 16884, and HD 181219). No proper motions were available for HD 23386 and no distance for SAO 45472.

In case of a spectroscopic binary, or when more than one radial velocity was available, a mean was adopted. This will introduce random scatter to the space motions of the newly discovered binaries because mostly only two velocities are available and their center-of-mass velocity remains undetermined. We thus plan to interpret these data with care.

The definitions and the computing procedure for the three space-motion components $(U, V, W)$ were outlined by Johnson $\&$ Soderblom (1987) and we basically follow their recommendations and adopt a right-handed galactic coordinate system. This makes our new values comparable to the values in the CABS catalog. The numerical values are listed in Tables A1 and A2. Again, we emphasize that for the cases of newly discovered binary systems, the observed radial velocity (or the average of the observed velocities in case the system is a SB2) are used in the computation of the space motions and not the (yet unknown) systemic velocities of the center of mass. These $U V W$-velocities are thus just first estimates. 
Table 3. New Doppler-imaging candidates

\begin{tabular}{llllllll}
\hline Star & $V$ & $\begin{array}{l}\Delta V_{\text {spot }} \\
(\mathrm{mag})\end{array}$ & $\begin{array}{l}\text { Sp. } \\
\text { type }\end{array}$ & SB? & $\begin{array}{l}P_{\text {phtm }} \\
(\text { days })\end{array}$ & $\begin{array}{l}v \sin i \\
\left(\mathrm{~km} \mathrm{~s}^{-1}\right)\end{array}$ & Notes \\
\hline HD 553 & 8.1 & 0.05 & K0III & SB1 & 9.06 & 38 & =V741 Cas, eclipsing \\
HIP 999 & 8.4 & 0.06 & G8V & SB1 & 1.84 & 20 & =LN Peg (=BD+13 13) \\
SAO 150676 & 9.0 & 0.07 & G0V+G6V & SB2 & 1.71 & 26 & G6-star \\
HD 291095 & 9.0 & 0.25 & K2-3IV & SB1 & 3.87 & 34 & $=$ =V1355 Ori, not in HIP \\
SAO 151224a & 9.3 & 0.15 & KIV+GIV & SB2 & 4.98 & 46 & eclipsing \\
HD 43989 & 8.5 & 0.03 & G0IV & S & 3.6 & 36 & $=$ =V1358 Ori, str. Li \\
HD 78644 & 8.2 & $?$ & G3V & SB1 & $?$ & 47 & no photometry \\
HD 82286 & 7.9 & 0.13 & K0IV & SB2 & 3.21 & $17 / 16$ & $=$ FF UMa, str. H $\alpha$ em. \\
HD 95559 & 9.0 & 0.08 & <G5V $>$ & SB2 & 2.94 & $31 / 26$ & possible triple \\
HD 106855 & 9.4 & 0.06 & K1V & SB2 & 2.04 & $13 / 16$ & $=$ UV Crv, H $\alpha$ em. \\
HD 109703 & 8.6 & 0.07 & G5III & S & 14.2 & 35 & very strong Li \\
HD 111487 & 9.0 & 0.10 & G5V & SB2 & 1.31 & 36 & $=$ IM Vir, eclipsing \\
HD 138157 & 7.1 & 0.12 & K0III & SB1 & 14.3 & 29 & $=$ OX Ser \\
HD 143937 & 8.6 & 0.10 & $<$ K0V $>$ & SB2 & 0.913 & $50 / 43$ & $=$ V1055 Sco, eclipsing \\
HD 145230 & 9.2 & 0.13 & K2IV & SB2 & 12.3 & 19 & $=$ PX Ser \\
HD 152178 & 8.1 & 0.10 & K0III & SB1 & 22.1 & 23 & $=$ V2253 Oph \\
HD 171488 & 7.4 & 0.07 & G2V & S & 1.337 & 36 & $=$ V889 Her, str. Li \\
HD 178450 & 7.8 & 0.10 & G8V & SB1 & 2.13 & 21 & $=$ =V478 Lyr \\
HD 190540 & 8.4 & 0.08 & K0III & SB1 & 17.7 & 19 & $=$ V4091 Sgr \\
HD 217352 & 7.2 & 0.04 & K2III & S & 18.4 & 35 & strong Li \\
HD 218153 & 7.6 & 0.10 & K0III & SB1 & 25.9 & 24 & $=$ KU Peg \\
\hline
\end{tabular}

\subsection{Rotational velocities}

Rotational velocities, $v \sin i$, for the stars with $\mathrm{Ca}$ II $\mathrm{H}$ and $\mathrm{K}$ emission were determined from the widths of selected lines in the red-wavelength spectra. The procedure includes a Gaussian or Voigt fit to several unblended line profiles (mostly Ni I $6643.63 \AA$ and Fe I $6663.45 \AA$ ) and the calibration of the average FWHM with $v \sin i$ according to the recipe of Fekel (1997). A mean macroturbulence profile and a (nightly) instrumental profile (approximated by a Gaussian) are subtracted from each FWHM measure according to Strassmeier et al. (1990). The following macroturbulence velocities were adopted: $3 \mathrm{~km} \mathrm{~s}^{-1}$ for solar-type dwarfs, $2 \mathrm{~km} \mathrm{~s}^{-1}$ for K dwarfs, $5 \mathrm{~km} \mathrm{~s}^{-1}$ for $<\mathrm{G} 5$ giants and subgiants, and $3 \mathrm{~km} \mathrm{~s}^{-1}$ for $>\mathrm{G} 5$ giants and subgiants (listed in Col. 9 in Table A1 and Table A2). Errors for $v \sin i$ from the red spectra are estimated to be $2-4 \mathrm{~km} \mathrm{~s}^{-1}$ for stars with $v \sin i<50 \mathrm{~km} \mathrm{~s}^{-1}$ and $\approx 5 \mathrm{~km} \mathrm{~s}^{-1}$ for stars with higher rotation rates.

For stars without a red spectrum, i.e. the stars without Ca II $\mathrm{H}$ and $\mathrm{K}$ emission, $v \sin i$ was estimated from the blue spectra by measuring the FWHM of two relatively unblended lines: the V I 4020.89- $\AA$ line and the Fe I 4087.80-Åline. The final values for $v \sin i$ in Table A2 were obtained from V I because this line turned out to be less prone to blending than Fe I 4087.80- $\AA$. Again, Fekel's (1997) relation was used for the transformation. The precision of $v \sin i$ from the blue spectra is comparably low due to the low $\mathrm{S} / \mathrm{N}$ ratio and spectral resolution and is of the order of $\pm 5-8 \mathrm{~km} \mathrm{~s}^{-1}$. A correlation of $v \sin i$ from V I $4020 \AA$ and Ni I $6643.63 \AA$ for the stars that had a blue and a red spectrum shows no systematic deviations above the expected measuring error and a rms of $5-8 \mathrm{~km} \mathrm{~s}^{-1}$.

The results for the standard stars $\beta$ Oph (K2III), $\alpha$ Ari (K2IIIab) and 35 Peg (K1III-IV) were $1.7 \pm 1.1 \mathrm{~km} \mathrm{~s}^{-1}$, $1.8 \pm 1.0 \mathrm{~km} \mathrm{~s}^{-1}$ and $1.5 \pm 1.2 \mathrm{~km} \mathrm{~s}^{-1}$, respectively, and compare well with the values listed in Fekel (1997) and references therein (i.e. $1.6-2.5 \mathrm{~km} \mathrm{~s}^{-1}, 1.8-3.1 \mathrm{~km} \mathrm{~s}^{-1}$, and $1.0 \mathrm{~km} \mathrm{~s}^{-1}$ for above stars, respectively).

\subsection{Light curves and rotation periods}

Table A3 presents the results from our photometric survey. Out of the 371 stars with Ca II H\& K emission only 172 could be observed because of telescope-time limitations but 168, i.e. $97.7 \%$, were found to be variable. A photometric period was determined for 134 of them, i.e. for $78 \%$ for the original 172 $\mathrm{H} \& \mathrm{~K}$ emission-line stars. Lower limits for the period are found for further 11 targets. Additionally, a total of 32 of our comparison or check stars turned out to be variables and for 22 of them a possible period, or a lower limit for the period, was obtained. All periods and full $y$ amplitudes in magnitudes are listed in Table A3 in Col. 7 and Col. 6, respectively. The periods are always given up to the last significant digit. Table A3 also gives information on the comparison and check star (Cols. 2 and 3), the JD of start and end of the observations (Cols. 4 and 5), the number of data points (Col. 8), and some individual notes (Col. 9). The full table is available electronically.

Except for a few eclipsing binaries (e.g. HD 553, HD 105575), we interpret the photometric periods found in this paper to be the stellar rotation period. Four of our target 
stars were recently discovered to be variables by Cutispoto et al. (1999). For two of these stars, we obtained a first period and confirmed the periods for the other two targets. Altogether, 170 new variables were discovered. The light curves in the figures are phased with the periods from Table A3 and a zero point in time of $T_{0}=2451000$. Figure B4 in the Appendix presents the light curves for stars that have a photometric period. Figure B5 in the Appendix shows the data for stars without a period.

We applied a program that performs a multiple frequency search through Fourier transforms with a non-linear leastsquares minimization of the residuals (Sperl 1998). The Fourier search range included a large number of frequencies up to the Nyquist frequency with a frequency spacing optimized for each individual data set. In most situations the frequency with the highest amplitude was adopted but, in some cases where the light curve appeared obviously double humped, twice the best-fit frequency was used. The best fits are determined by minimizing the squares of the residuals between trial fits and measurements. Further details on the period analysis can be found in Strassmeier et al. (1999).

To judge the significance of certain frequency peaks we compute a running mean of the frequency distribution for a signal-to-noise ratio of 4:1 which was found empirically by Breger et al. (1993) to indicate the limit for a significant period. From numerical simulations with varying amounts of white noise, Kuschnig et al. (1997) showed that frequency peaks with $S / N \geq 4.0$ suggest a $99.9 \%$ probability for a real period. Furthermore, since we interpret the photometric period to be the stellar rotation period, a particular period must be in agreement with the spectroscopically measured $v \sin i$ and an assumed stellar radius according to the adopted spectral classification. This reduces the range of possible periods significantly.

\section{Summary}

Figure 4 summarizes the statistics of the survey results. A series of histograms show the distribution of the numerical values of nine activity indicators. Out of the total of 1058 stars observed, $371(35 \%)$ were found with Ca II H\&K emission but only 78 (7.3\%) with $v \sin i \geq 10 \mathrm{~km} \mathrm{~s}^{-1}$. On the contrary, a lithium line was detected in 283 (74\%) of all stars that had Ca II emission (with $58 \%$ of the stars with lithium above $10 \mathrm{~m} \AA$ ). Out of a subsample of 172 stars with moderate to strong Ca II emission, $168(97.7 \%)$ turned out to be photometric variables and for 134 a photometric (i.e. rotation) period could be obtained. Stellar activity, rotational broadening of spectral lines, and knowledge of a precise stellar rotation period are the key requirements for Doppler imaging. Table 3 lists our new candidates for observation with this technique. Three of the candidates, HD 218153 , HD 171488 and HD 291095, were already observed with highresolution spectroscopy and are currently being Doppler imaged by the authors. In a forthcoming second part of the analysis, we will consider relations between the various activity indicators as deduced from the observations in this paper.
Acknowledgements. KGS is very grateful to the Austrian Fond zur Förderung der wissenschaftlichen Forschung for support through grants S7302-AST (Doppler imaging) and S7301-AST (APT). We all wish to thank NOAO/KPNO for devoting generous amounts of coudé-feed telescope time to fundamental spectroscopy of active stars. We also appreciate numerous discussions with Dr. F.C. Fekel (TSU) regarding our targets and with Dr. J.B. Rice regarding the applicability of the Doppler-imaging technique. This research has made extensive use of the Simbad database, operated at CDS, Strasbourg, France.

\section{References}

Balona L.A., 1987, SAAO Circ. 11, 1

Bidelman W.P., 1954, ApJS 1, 175

Bidelman W.P., 1981, AJ 86, 553

Bidelman W.P., 1983, AJ 88, 1182

Bidelman W.P., 1985, AJ 90, 341

Bidelman W.P., 1988, PASP 100, 1084

Bidelman W.P., MacConnell D.J., 1973, AJ 78, 687

Breger M., Stich J., Garrido R., et al., 1993, A\&A 271, 482

Buzasi D.L., 1999, in Pallavicini R. (ed.), Stellar Clusters and associations: convection, rotation, and dynamos, Euroconference, Palermo, PASPC (in press)

Cutispoto G., Pastori L., Tagliaferri G., Messina S., Pallavicini R., 1999, A\&AS 138, 87

Duncan D.K., Vaughan A.H., Wilson O.C., et al., 1991, ApJS 76, 383

ESA, 1997, The Hipparcos and Tycho catalogue, SP-1200

Fekel F.C., 1997, PASP 109, 514

Fekel F.C., Balachandran S., 1993, ApJ 403, 708

Fekel F.C., Moffett T.J., Henry G.W., 1986, ApJS 60, 551

Fekel F.C., Strassmeier K.G., Weber M., Washuettl A., 1999, A\&AS 137,369

Flower P.J., 1996, ApJ 469, 355

Gray D.F., 1992, The observation and analysis of stellar photospheres. CUP, Cambridge, p. 430

Gray R.O., Garrison R.F., 1989, ApJS 69, 301

Grevesse N., Anders E., 1991, in Cox A.N., et al. (eds.), Solar Interior and Atmosphere. The Univ. of Arizona Press, Tucson, p. 1227

Hall J.C., 1996, PASP 108, 313

Henry T.J., Soderblom D.R., Donahue R.A., Baliunas S.L., 1996, AJ 111,439

Johnson D.H., Soderblom D.R., 1987, AJ 93, 864

Kaye A.B., Strassmeier K.G., 1998, MNRAS 294, L35

Kuschnig R., Weiss W.W., Gruber R., Bely P.Y., Jenkner H., 1997, A\&A 328, 544

Lastennet E., Freire Ferrero R., 1994, A\&AS 108, 611

Linsky J.L., Worden S.P., McClintock W., Robertson R.M., 1979, ApJS 41, 47

Lloyd-Evans T., Koen M.C.J., 1987, SAAO Circ. 11, 21

Maitzen H.M., Paunzen E., Pressberger R., Slettebak A., Wagner R.M., 1998, A\&A 339, 782

Milone E.F., Naftilan S.A., 1980, in Plavec M.J., Popper D.M., and Ulrich R.K. (eds.), Close Binary Systems: Observations and Interpretations, IAU Symp. 88. Reidel, Dordrecht, p. 419

Noyes R.W., Hartmann L.W., Baliunas S.L., Duncan D.K., Vaughan A.H., 1984, ApJ 279, 763

Pavlenko Ya.V., Magazzù A., 1996, A\&A 311, 961

Rice J.B., 1996, in Strassmeier K.G. \& Linsky J.L. (eds.), IAU Symp. 176, Stellar Surface Structure. Kluwer, Dordrecht, p. 19 
Rutten R.G.M., Schrijver C.J., Lemmens A.F.P., Zwaan C., 1991, A\&A 252, 203

Scarfe C.D., Batten A.H., Fletcher J.M., 1990, Publ. Dominion Ap. Obs. 18 , No. 2, 21

Schrijver C.J., 1996, in Strassmeier K.G. \& Linsky J.L. (eds.), IAU Symp. 176, Stellar Surface Structure. Kluwer, Dordrecht, p. 1

Soderblom D.R., Jones B.F., Balachandran S., Stauffer J.R., Duncan D.K., Fedele S.B., Hudon J.D., 1993, AJ 106, 1059

Soderblom D.R., King J.R., Henry T.J., 1998, AJ 116, 396

Solanki S., Motamen S., Keppens R., 1997, A\&A 324, 943

Sperl M., 1998, Commun. Astroseismology 111, 1

Stix M., 1989, The Sun, A\&A Library. Springer, Berlin

Strassmeier K.G., 1994, A\&AS 103, 413

Strassmeier K.G., Bartus J., Kövari Z., Weber M., Washuettl A., 1998, A\&A 336, 587

Strassmeier K.G., Bartus J., Rodonó M., Cutispoto G., 1997a, A\&AS 125,11

Strassmeier K.G., Boyd L.J., Epand D.H., Granzer Th., 1997b, PASP 109,697

Strassmeier K.G., Fekel F.C., Bopp B.W., Dempsey R.C., Henry G.W., 1990, ApJS 72, 191

Strassmeier K.G., Hall D.S., Fekel F.C., Scheck M., 1993, A\&AS 100, 173

Strassmeier K.G., Handler G., Paunzen E., Rauth M., 1994, A\&A 281, 855

Strassmeier K.G., Serkowitsch E., Granzer Th., 1999, A\&AS 140, 29

Willstrop R.V., 1964, MRAS 69, 83

Wilson O.C., 1976, ApJ 205, 823

\section{Appendix A: Tables}

Table A1 lists the results for stars that were found to exhibit $\mathrm{H} \& \mathrm{~K}$ emission while Table A2 lists the results for stars without detectable H\&K emission. Table A3 summarizes the results from the photometric monitoring. All tables are available only in electronic form.

\section{Appendix B: Figures}

The following five figures show the spectra for the H\&K emission-line stars in the wavelength region around $\mathrm{Ca}$ II $\mathrm{H}$ and $\mathrm{K}$ (Fig. B1). A subsample of the stars with strong Ca II emission is plotted in the $\mathrm{H} \alpha$ region (Fig. B2) and in the lithium region at $6708 \AA$ (Fig. B3). The Strömgren $y$ photometry is shown in Figs. B4 and B5 (Fig. B4 is for the stars with a photometric period and Fig. B5 for those without a detectable period).

\section{Next File}



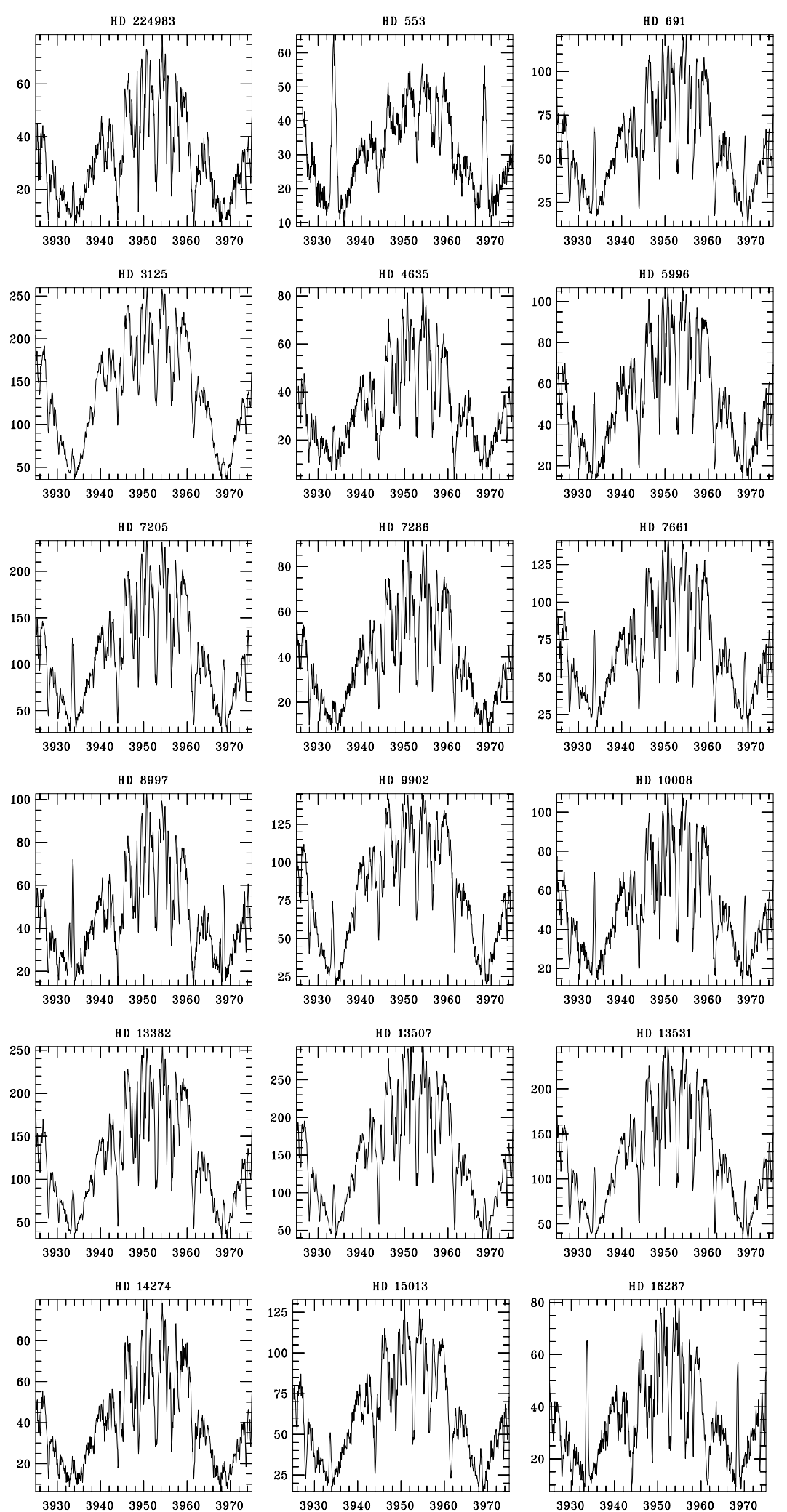

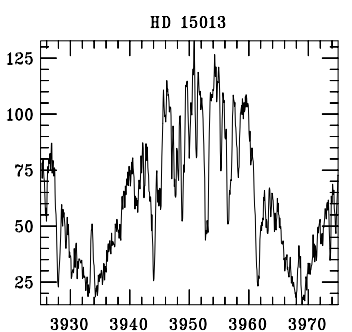

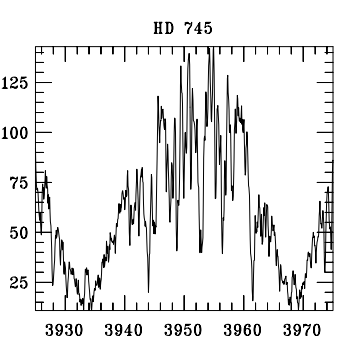
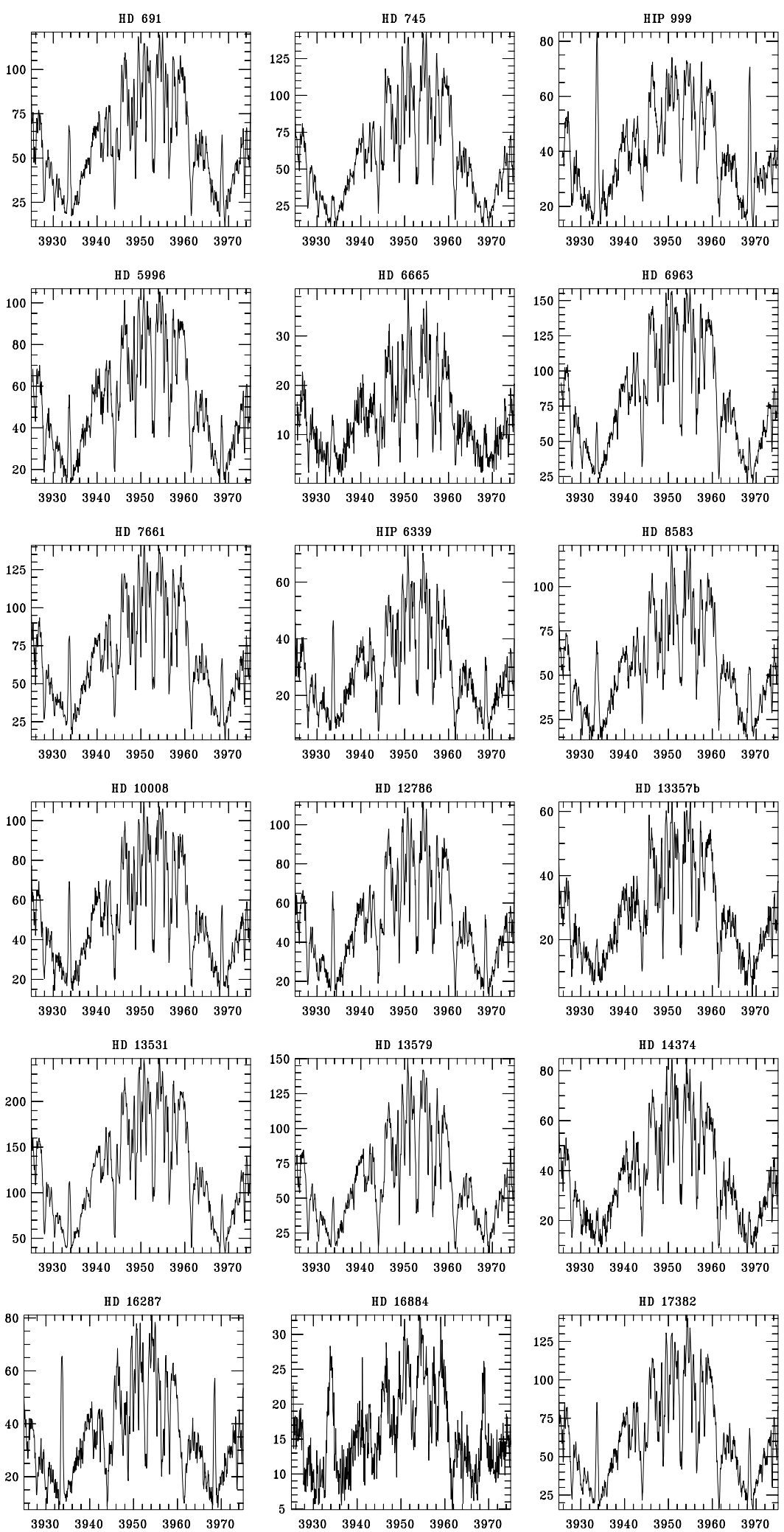

Fig. B1. Ca II spectra of stars with $\mathrm{H}$ and $\mathrm{K}$ emission. Shown are the 316 stars with an intensity level in the K line of $I_{\mathrm{K}} \geq 1$. The HD number (or HIP number or Variable Star designation if no HD number exists) is identified on top of each plot. Each plot covers a range of $50 \AA \AA$ centered at $3950 \AA$ 


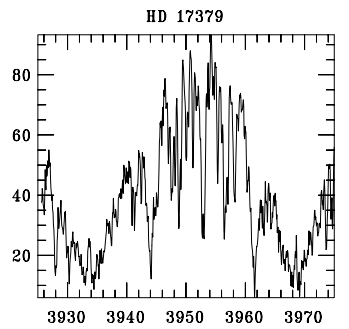

HD 19668

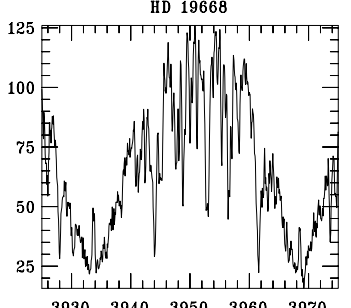

HD 21845

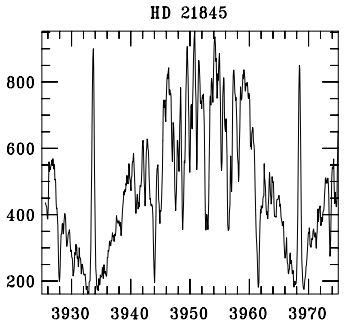

(1750
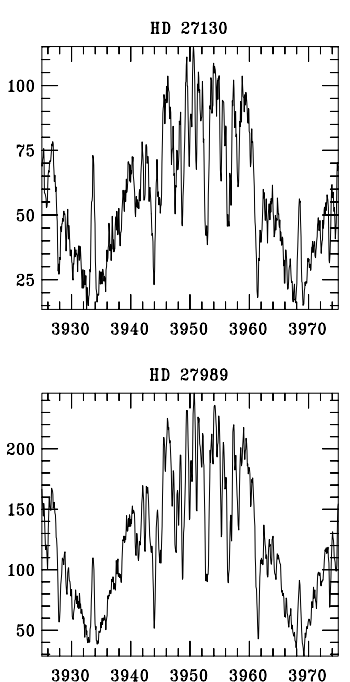

HD 37216

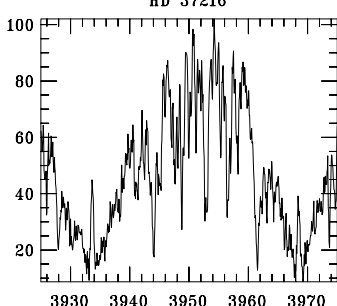

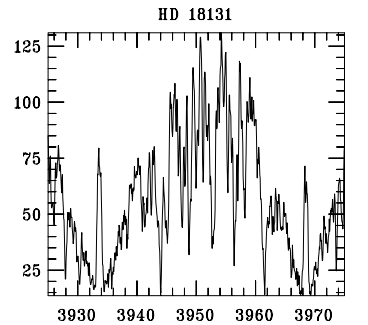

HD 19902
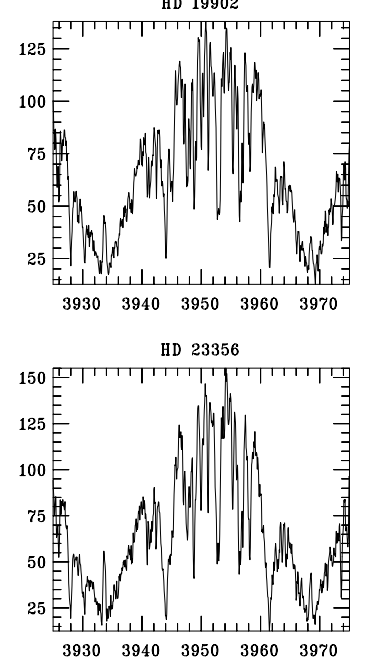

HD 25998
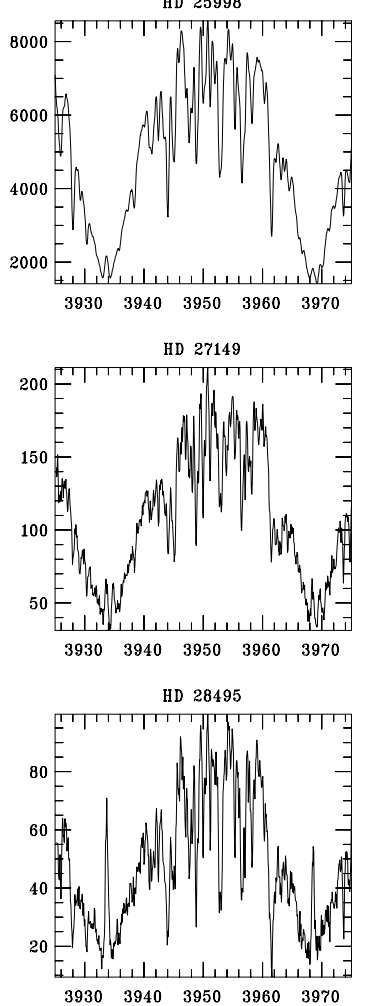

SA0 150676

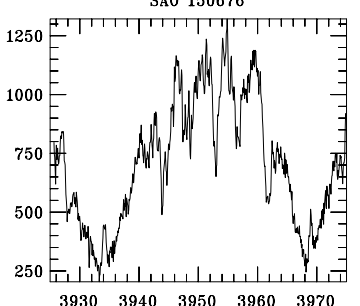

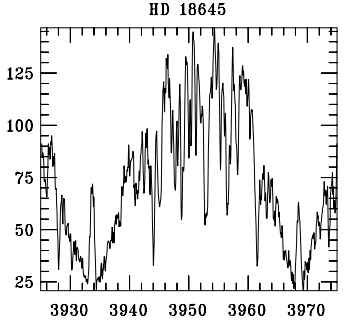

HD 19942

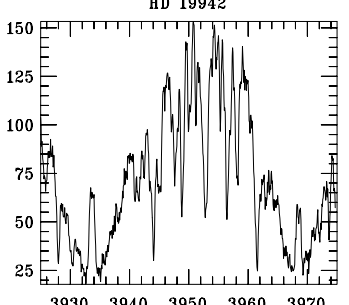

HD 23140
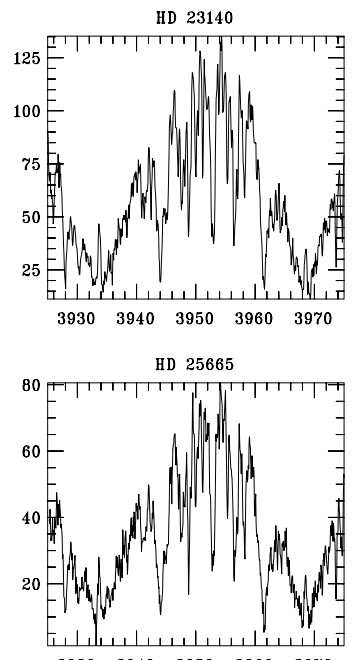

$\begin{array}{lllll}3930 & 3940 & 3950 & 3960 & 3970\end{array}$
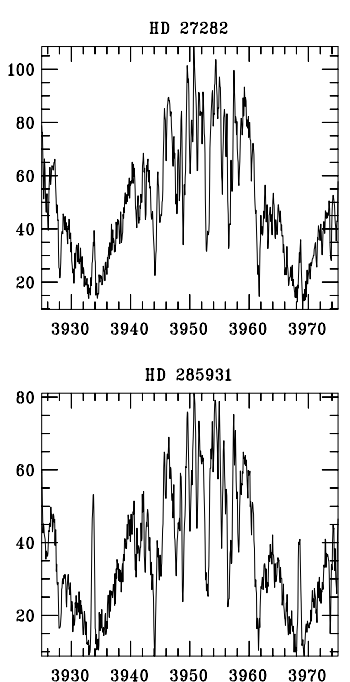

HD 41067

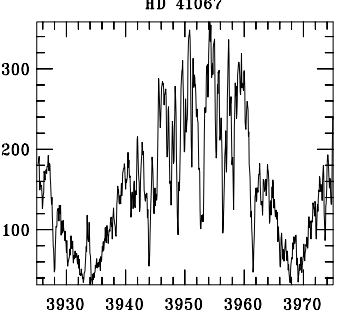

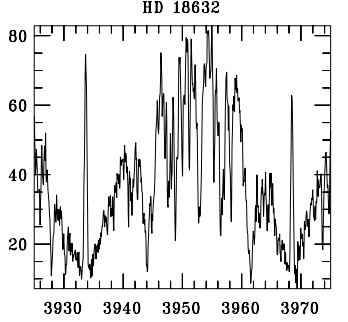

HD 20678
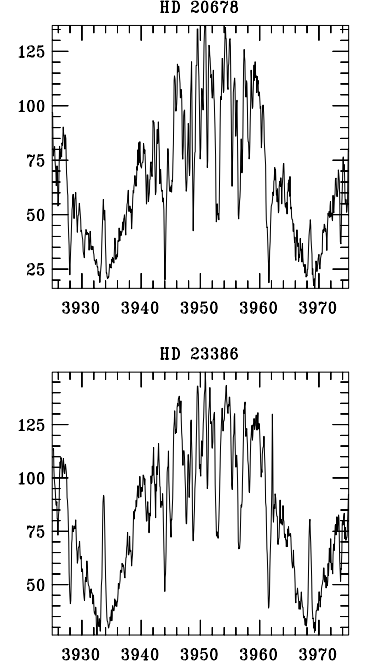

(1)
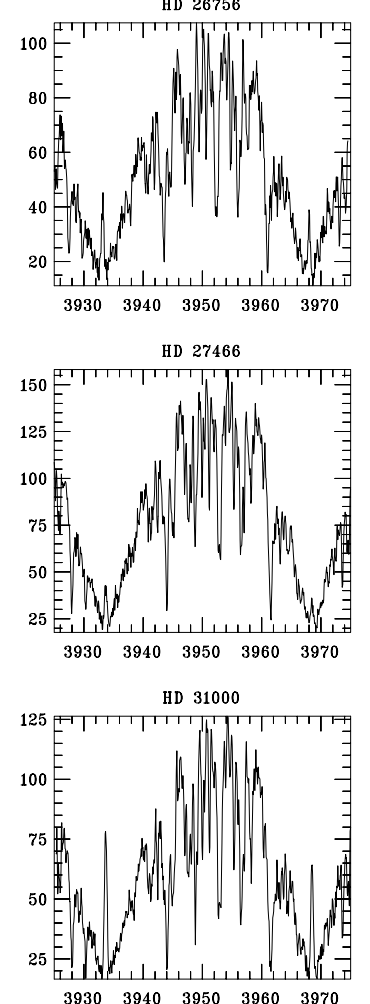

HD 291095

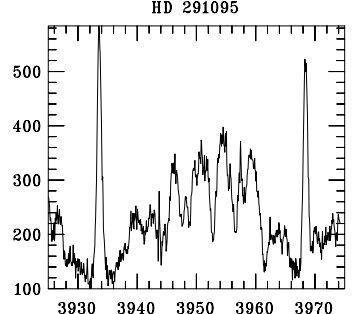

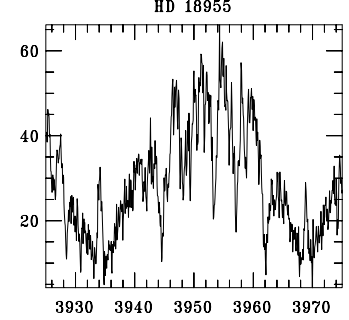
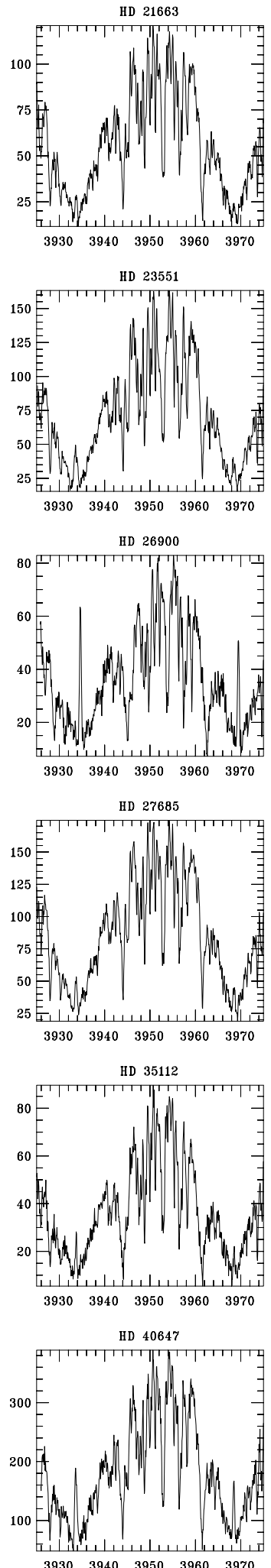

Fig. B1. continued 

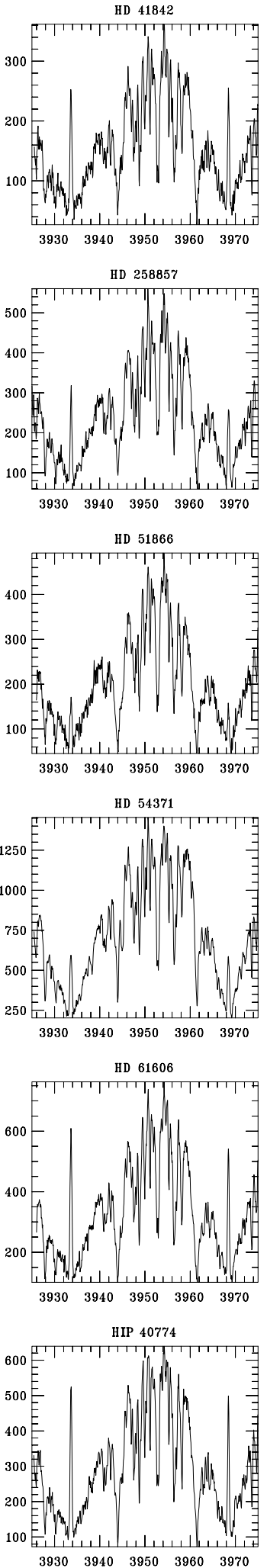

HD 71251

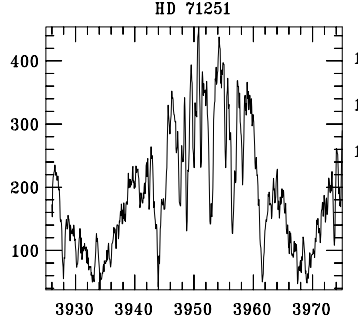

Fig. B1. continued
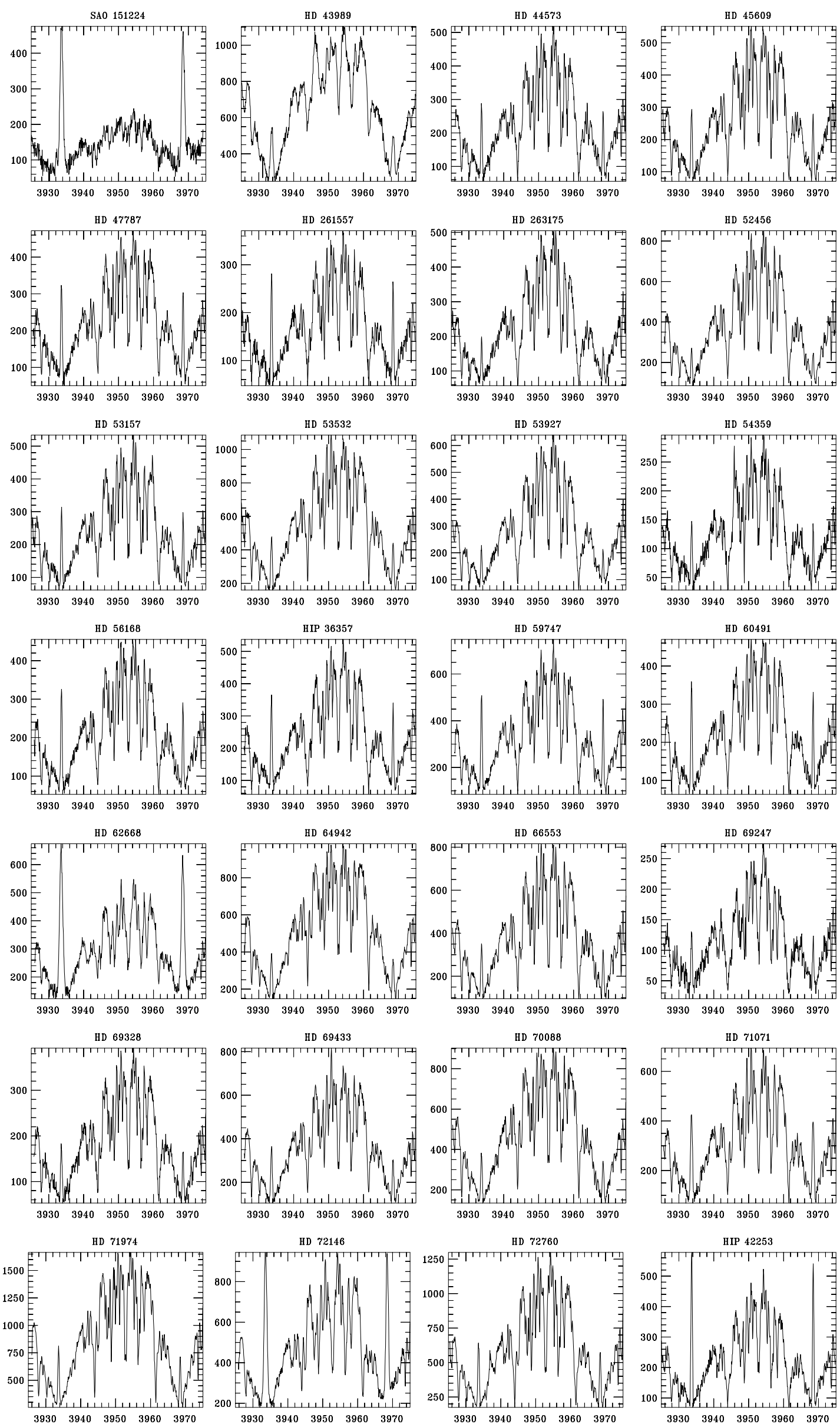

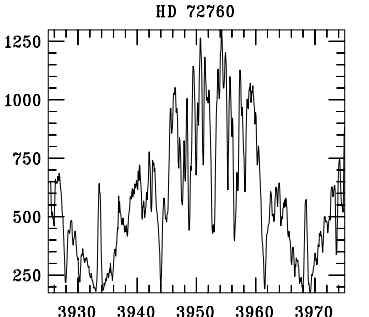

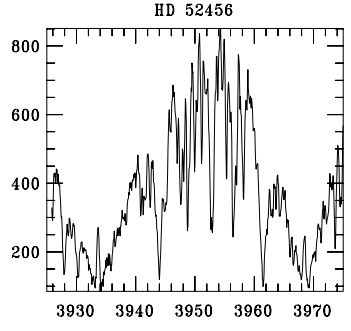
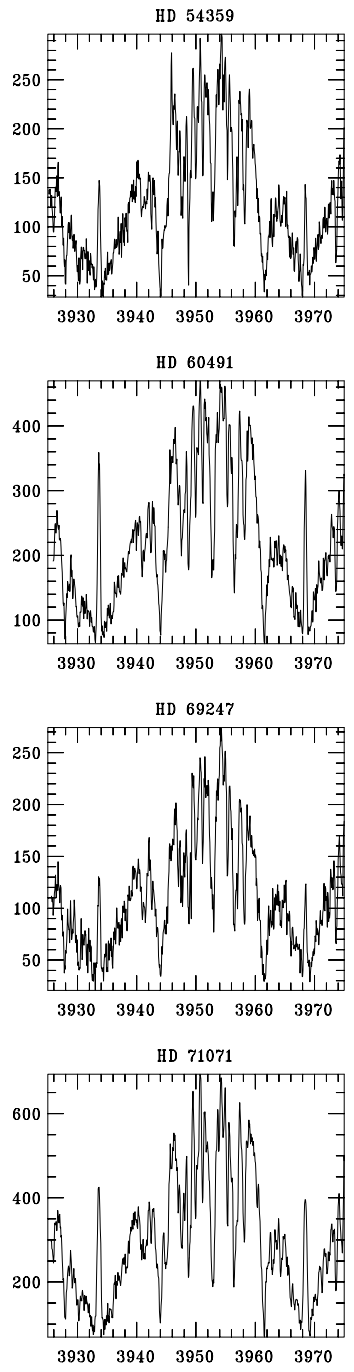

HIP 42253

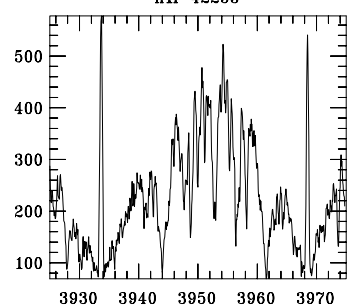



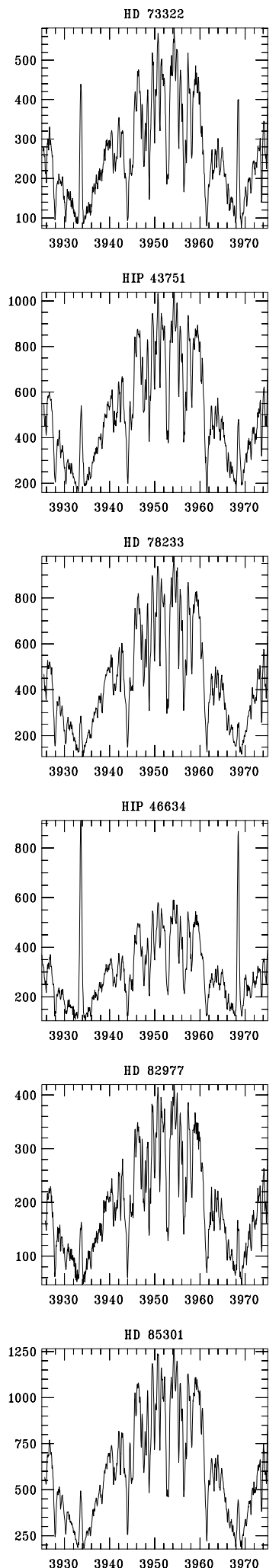

HD 88654

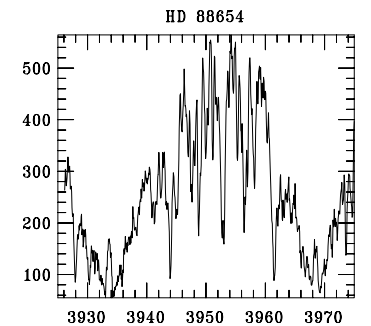

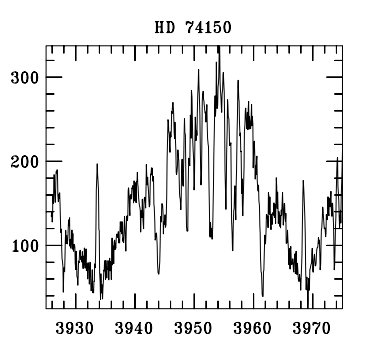
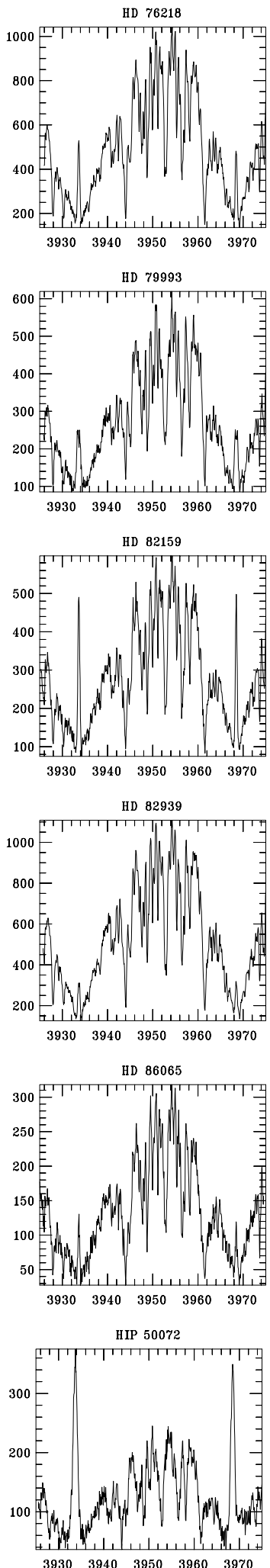

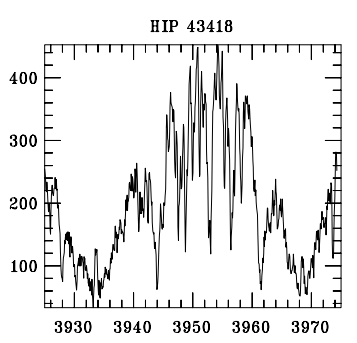

HD 76799
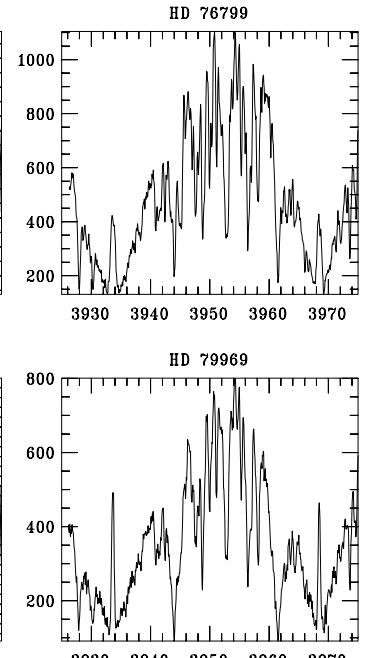

$\begin{array}{llllll}3930 & 3940 & 3950 & 3960 & 3970\end{array}$
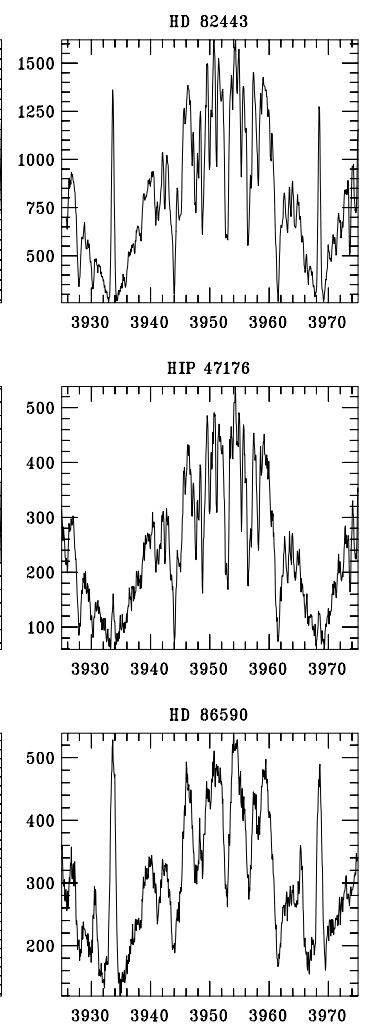

HD 88638

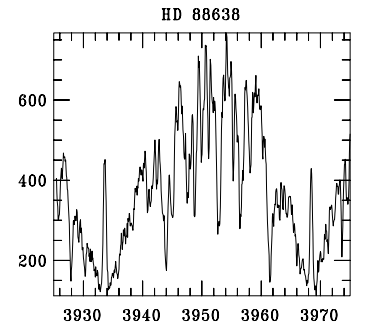

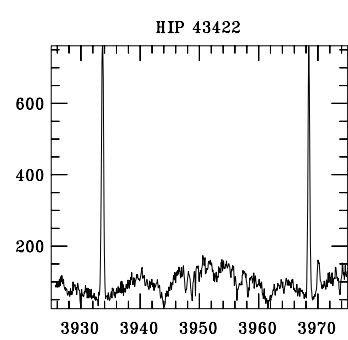
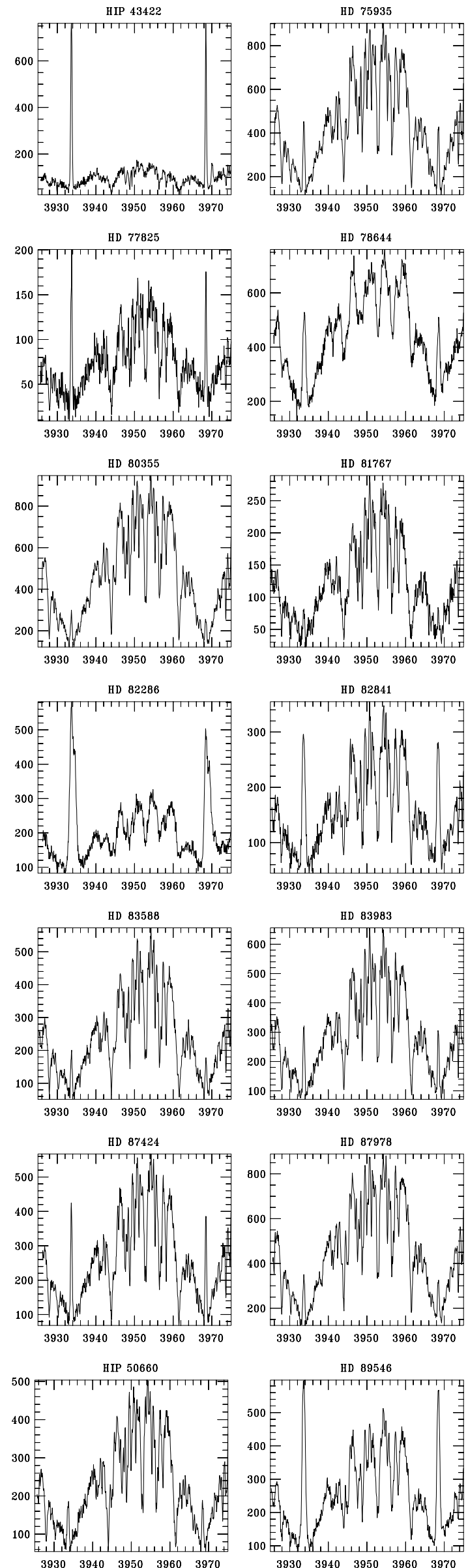
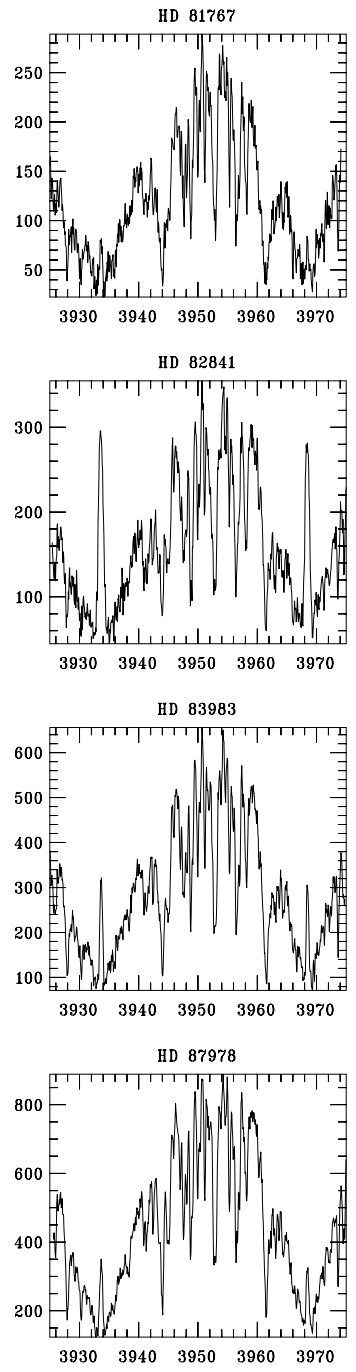

HD 89546

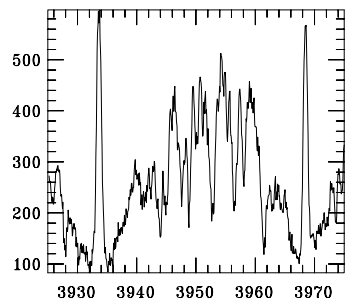

Fig. B1. continued 

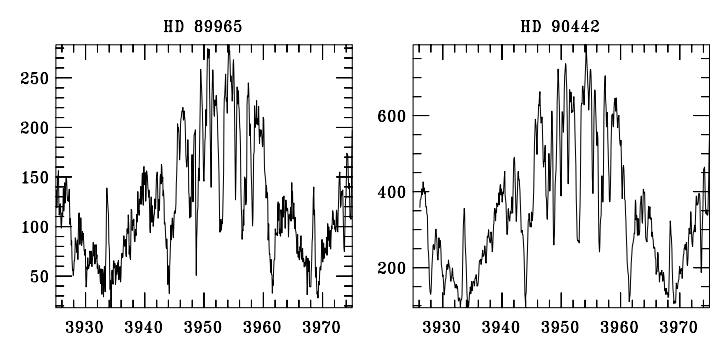

HD 93811

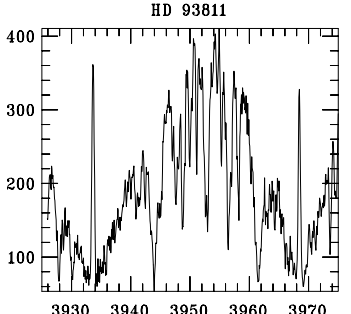

HD 95559
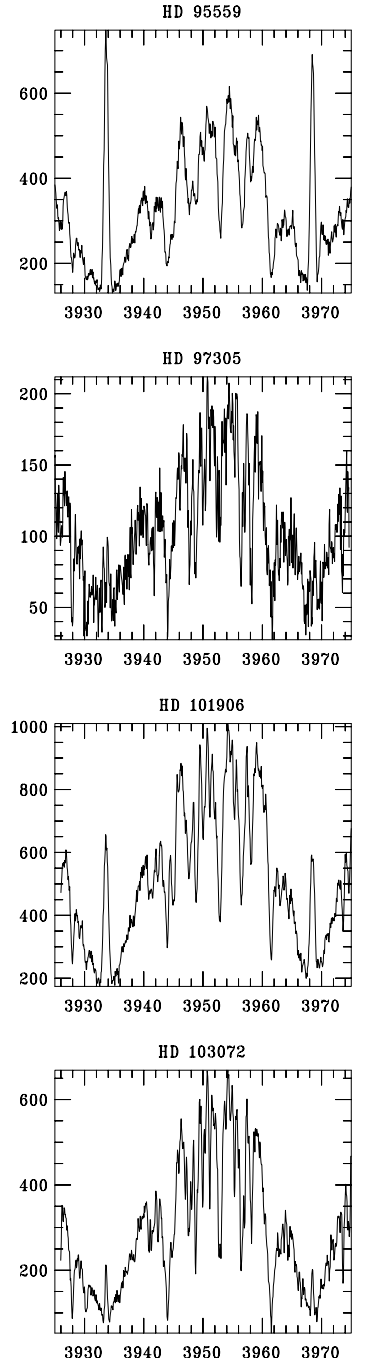

HD 104923

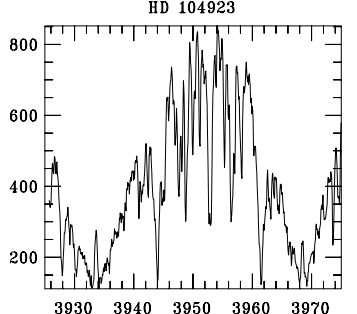

D 9391
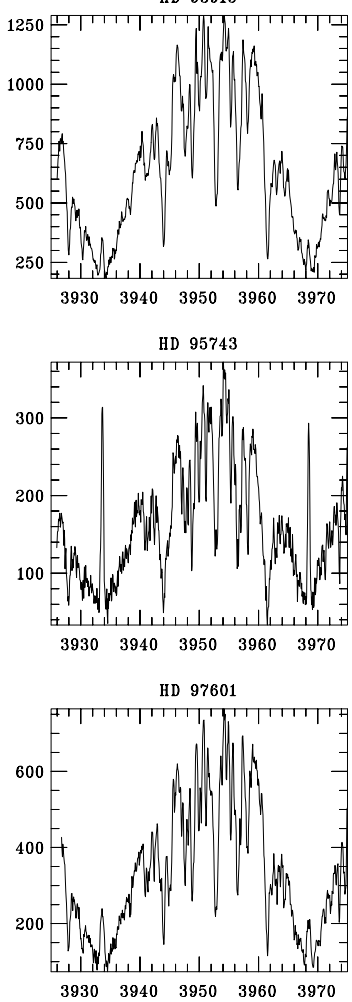

$$
\text { HD } 102121
$$
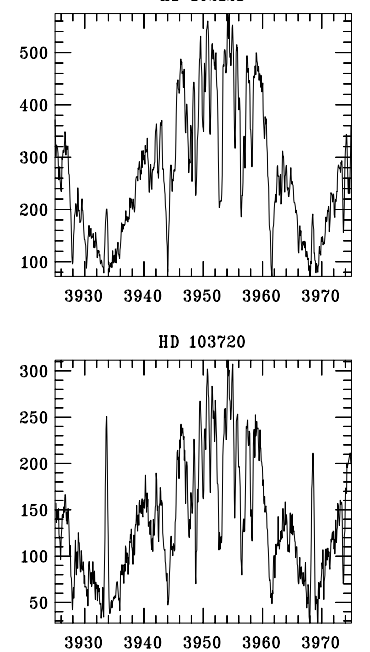

HIP 59152

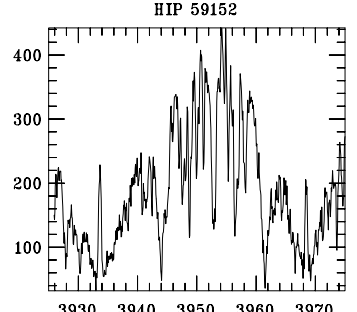

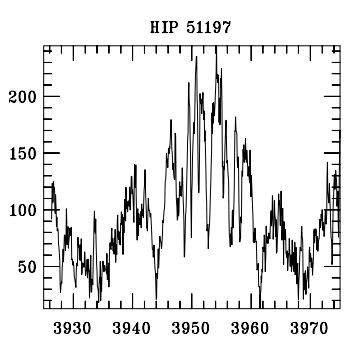
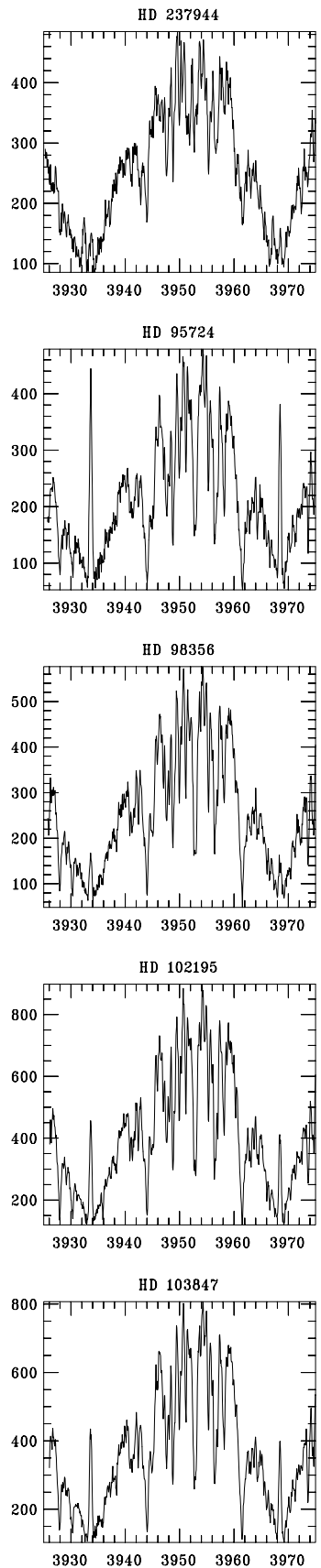

HD 105575

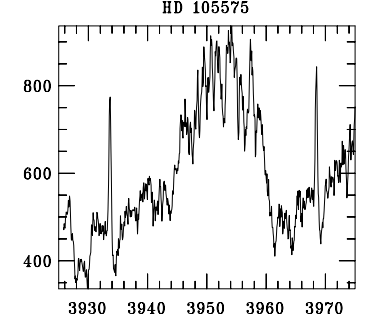

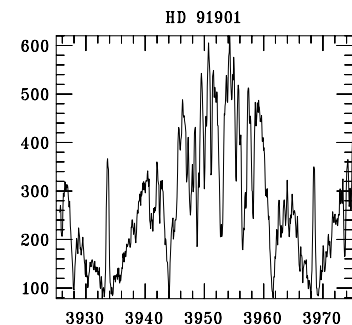
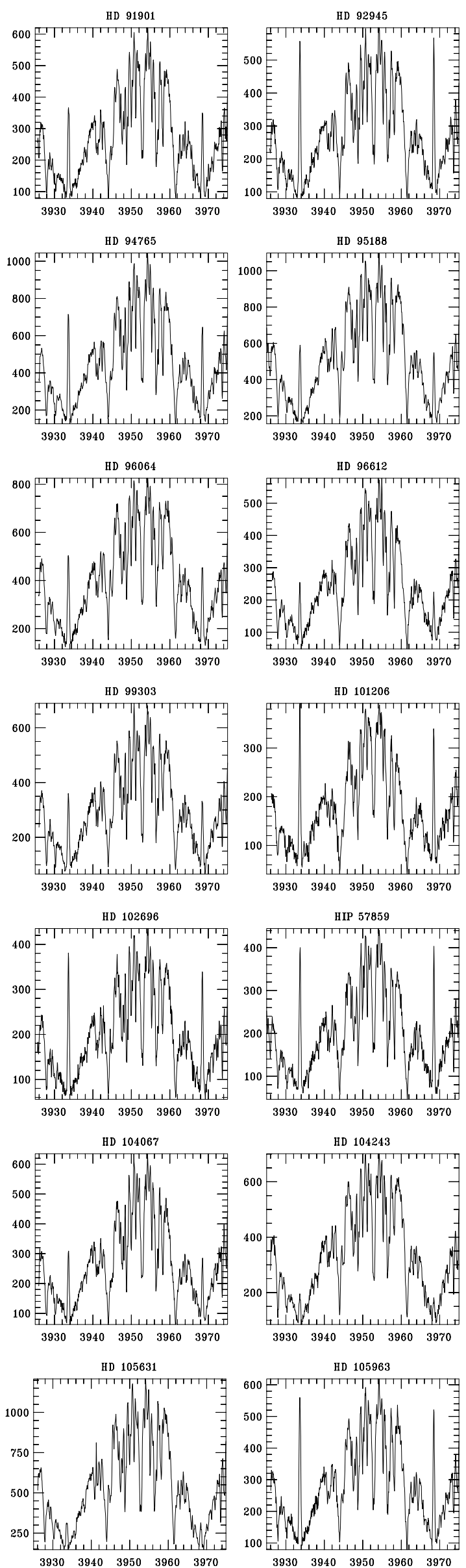

Fig. B1. continued 

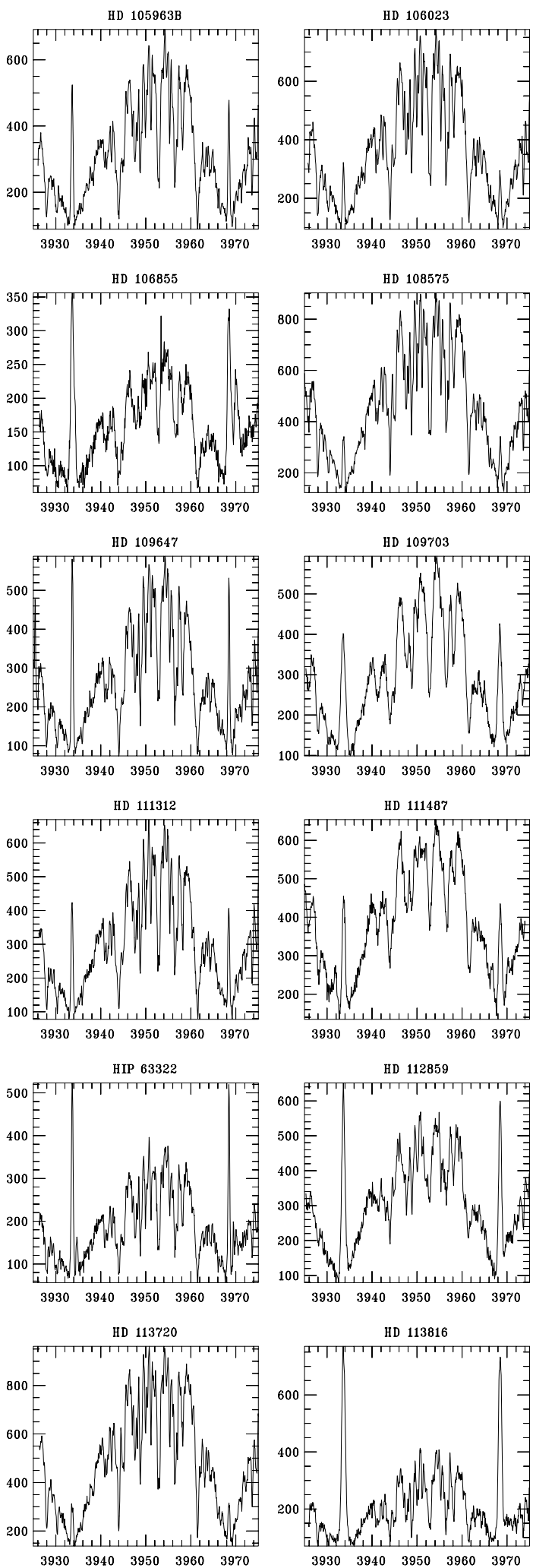

HD 117860

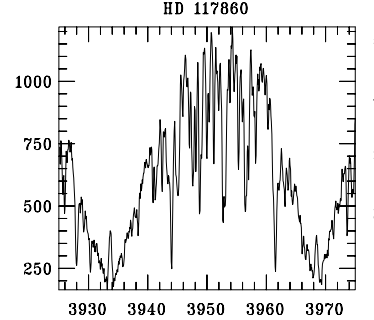

Fig. B1. continued
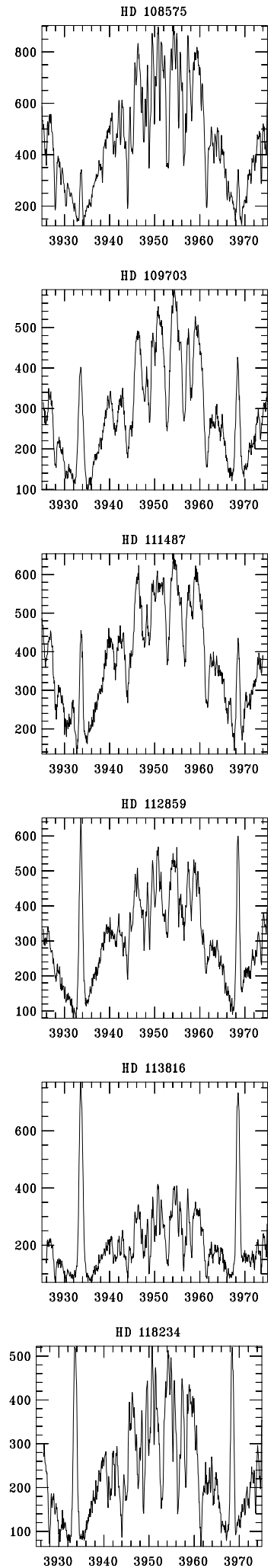
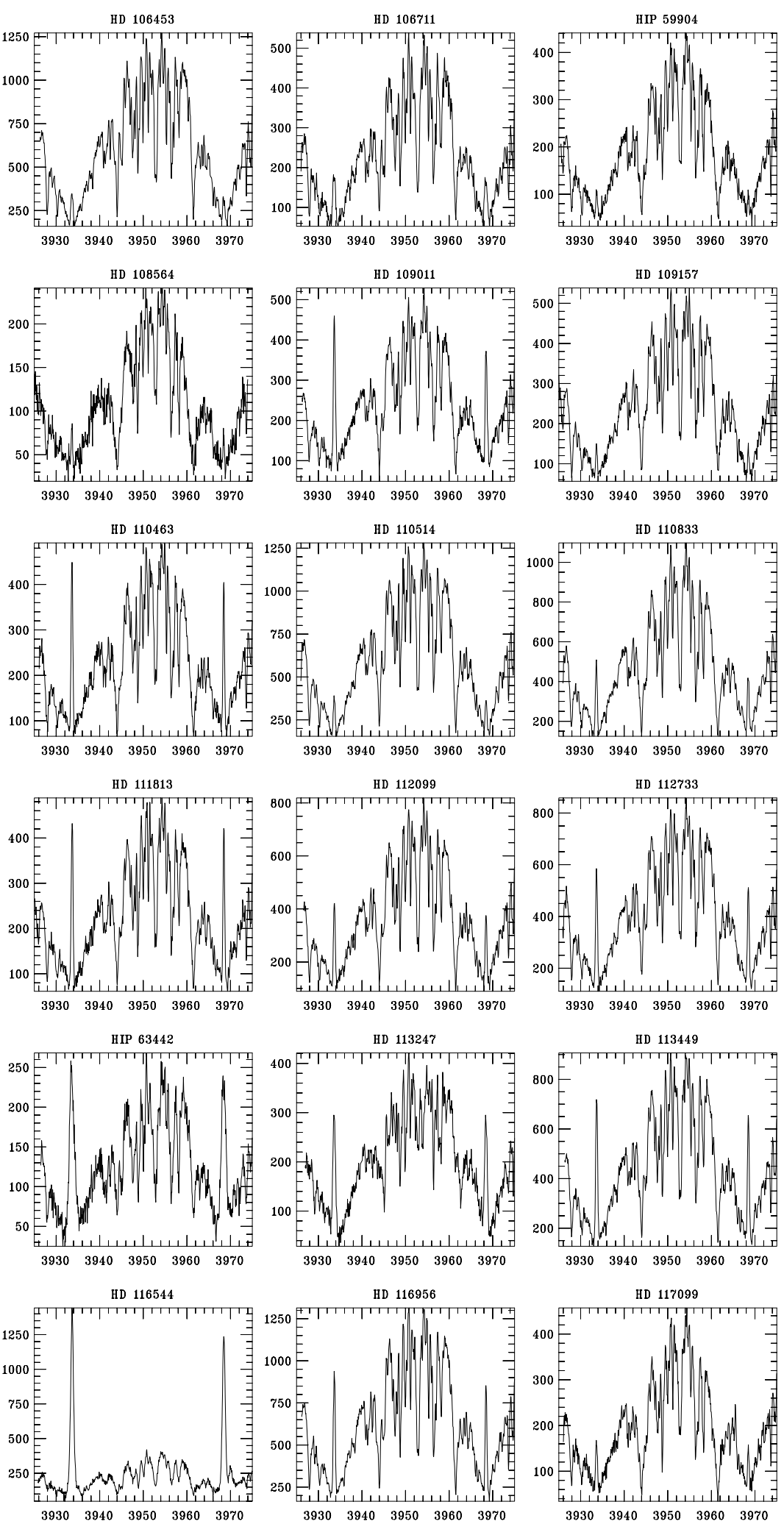

HD 119332
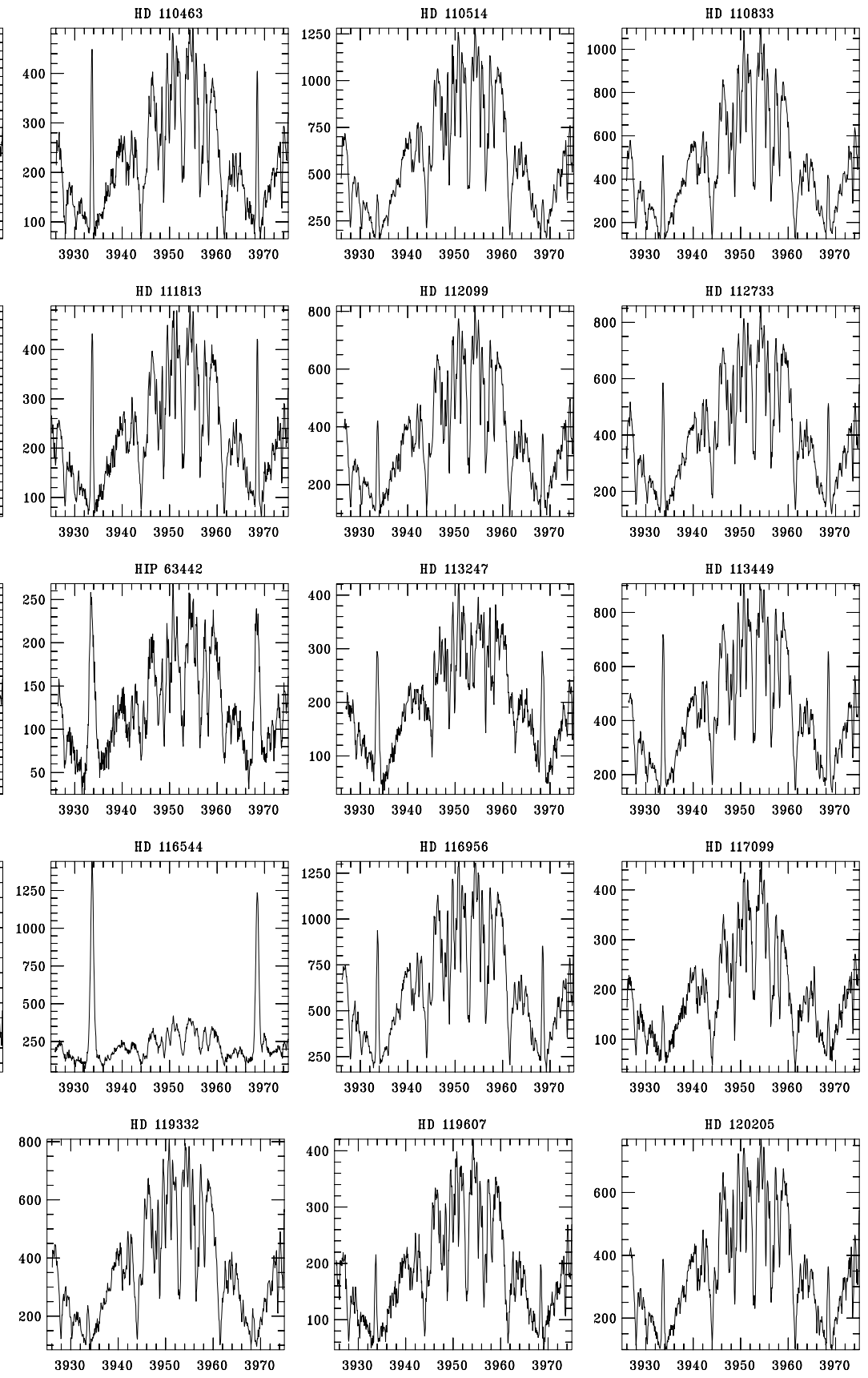


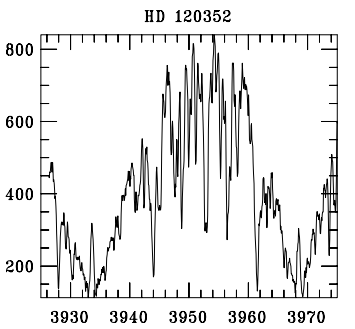

HD 123351

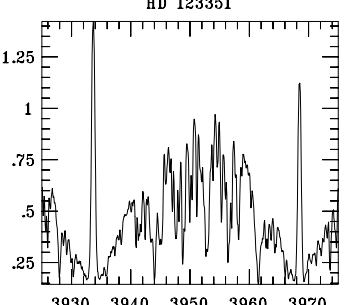

HD 127068

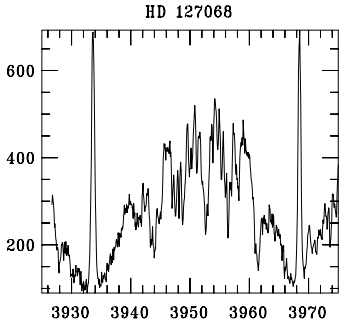

HD 130004
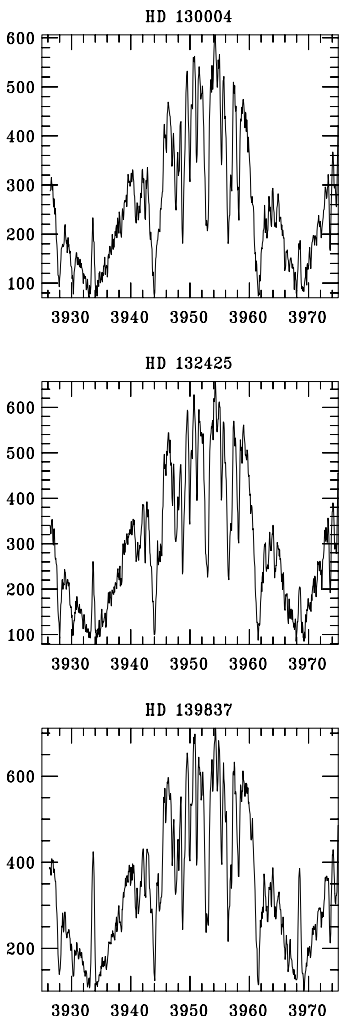

HD 141272

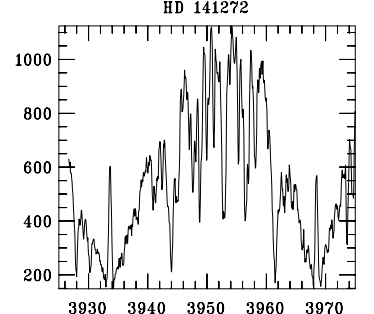

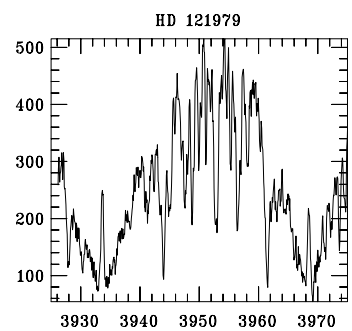

HD 124106
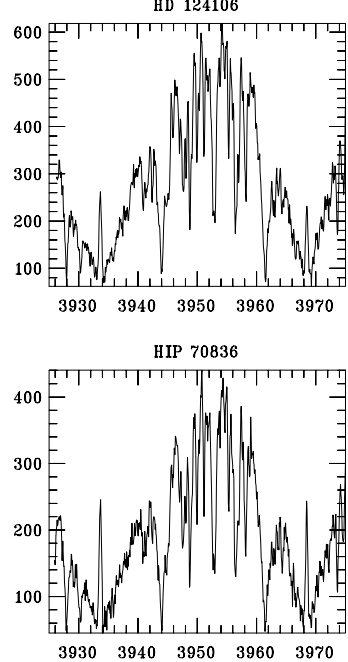

HD 130215
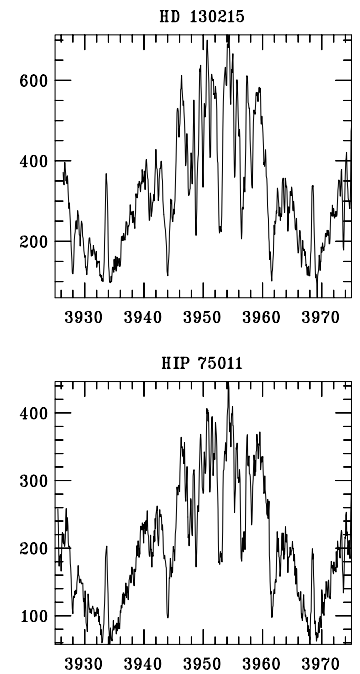

$$
\text { HIP } 77179
$$

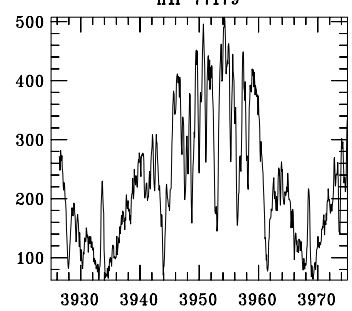

HD 141919

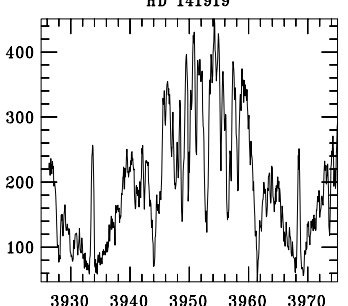

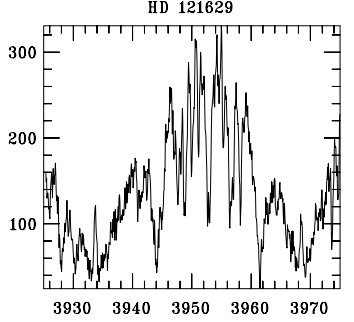
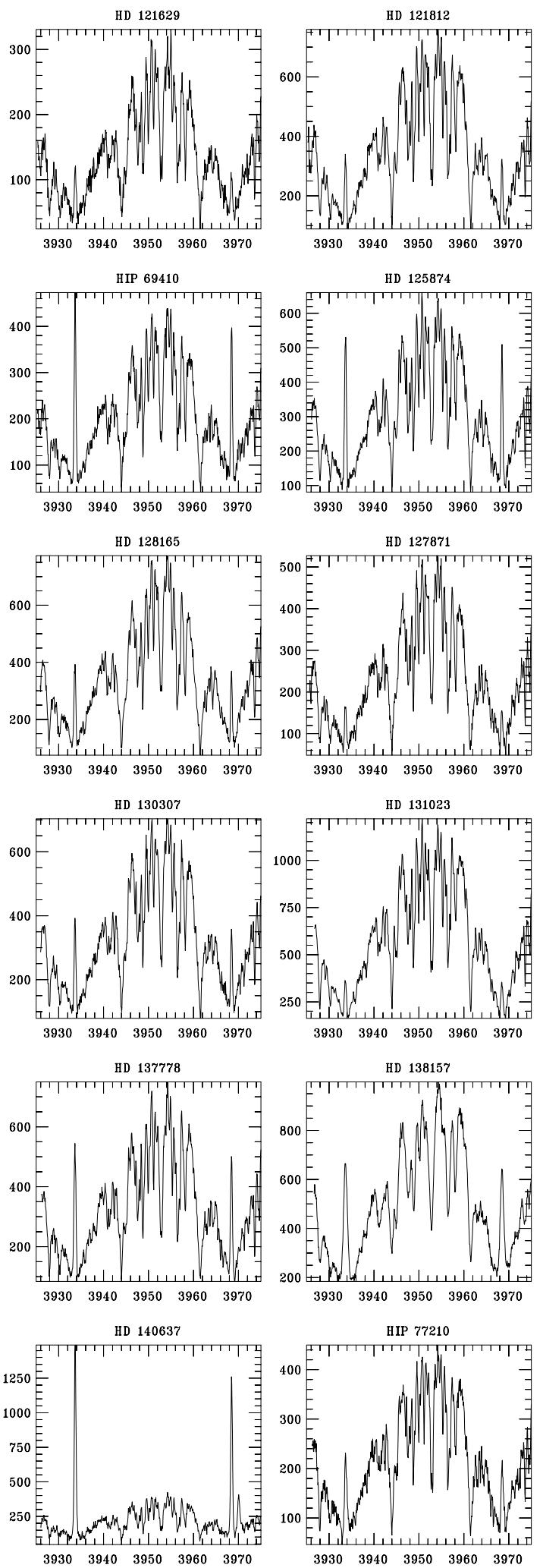

HD 142072

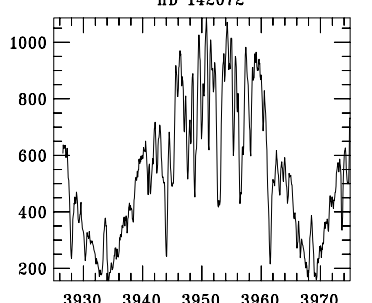

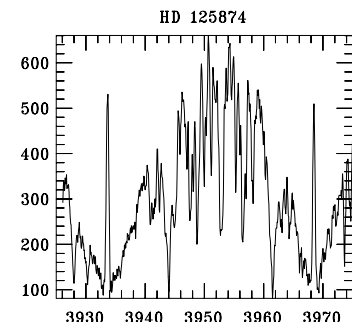
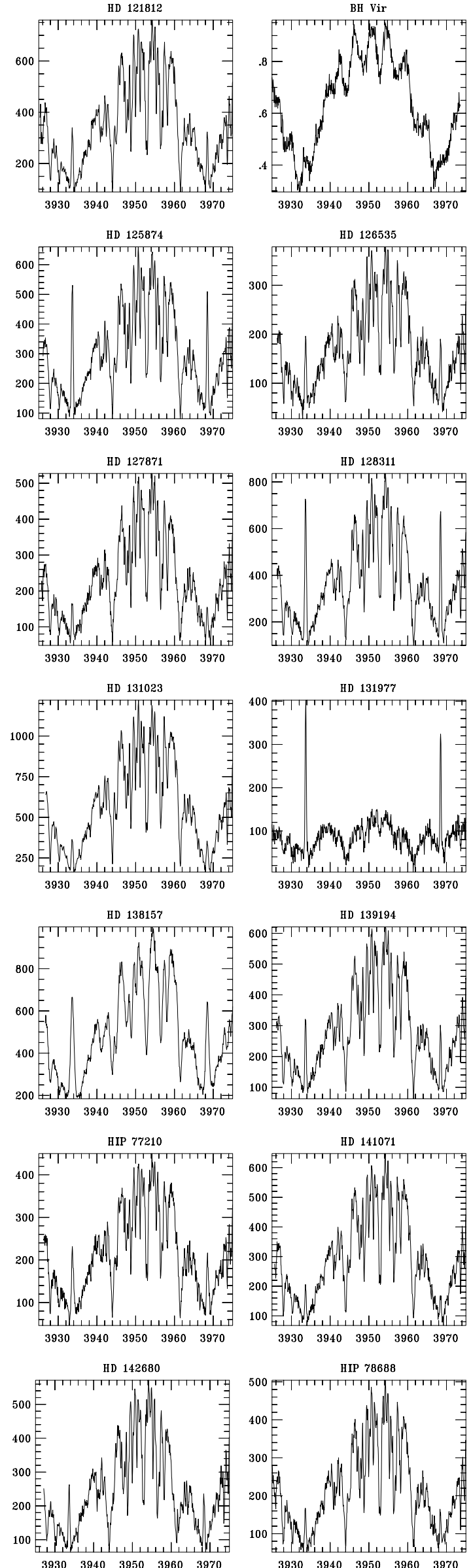

Fig. B1. continued 


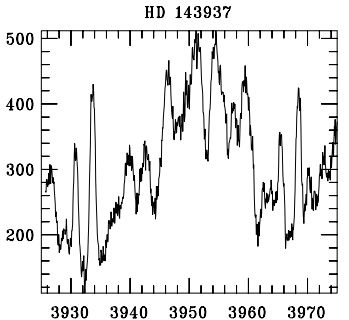

HD 147776

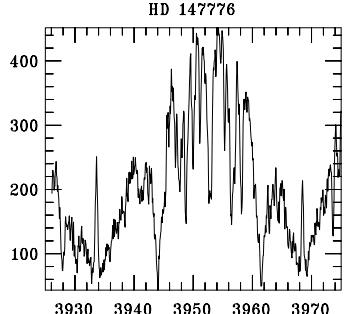

HD 152178
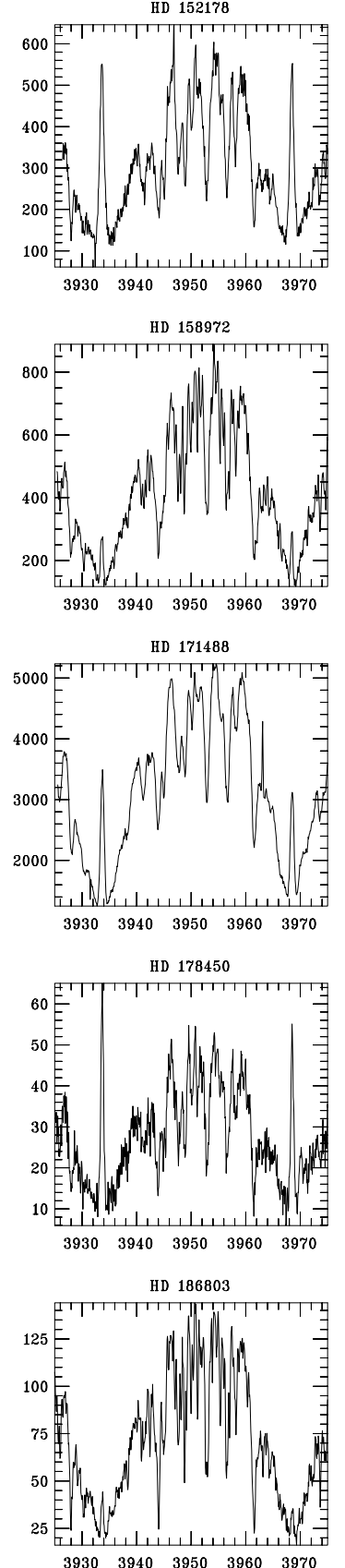

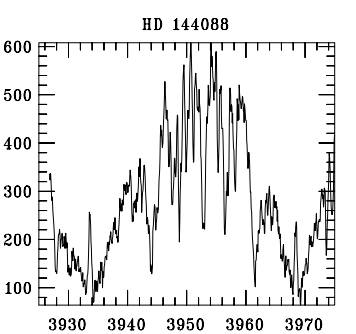

HD 149028
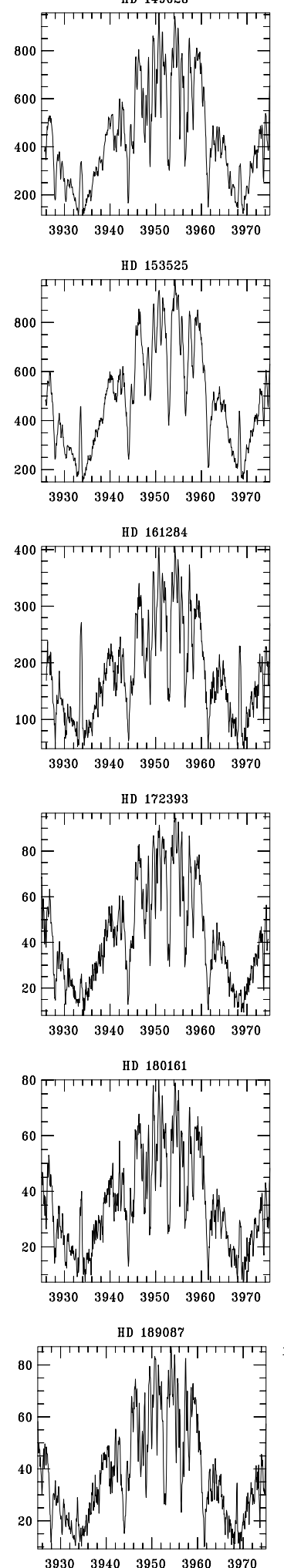
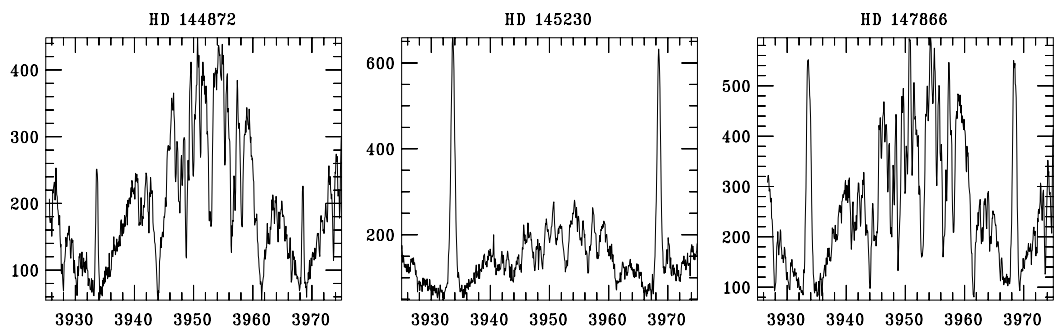

HD 150748
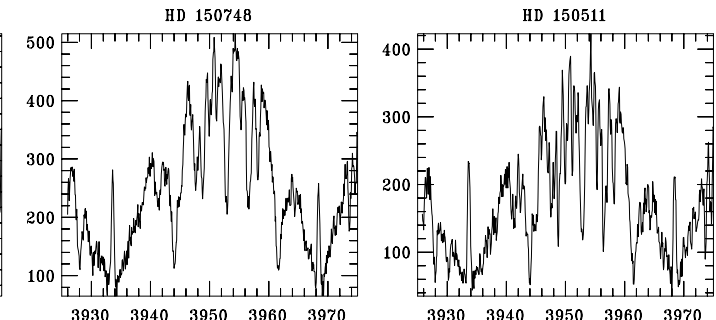

HD 153557

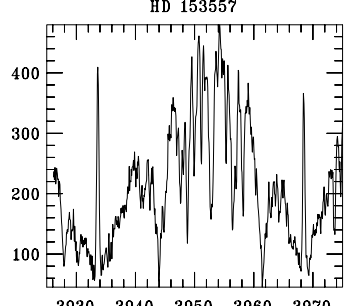

$\begin{array}{llllll}3930 & 3940 & 3950 & 3960 & 3970\end{array}$

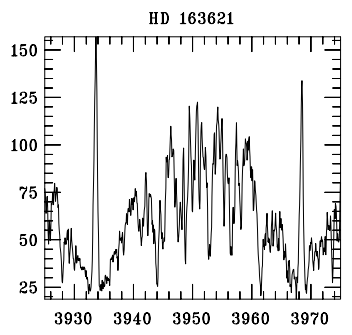

HD 173950
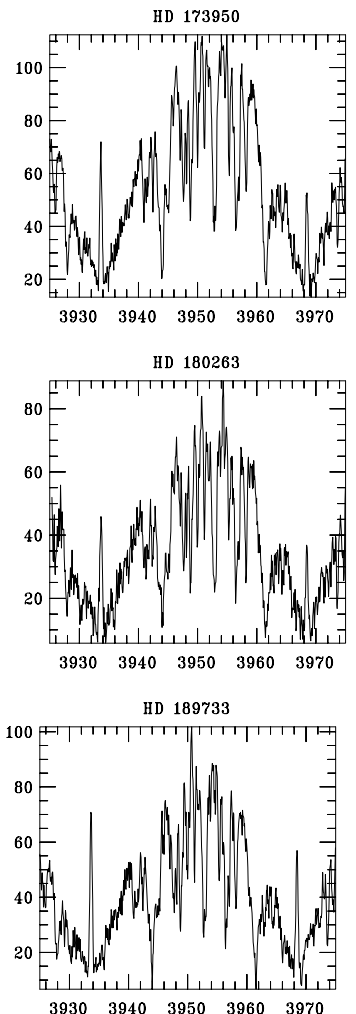

HIP 83141

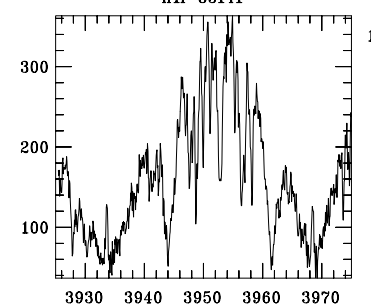

HD 167715

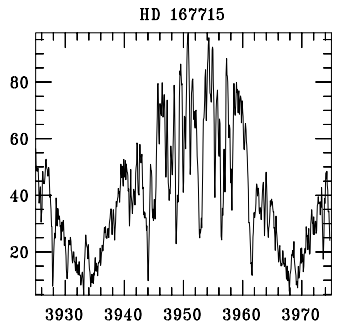

HD 175742
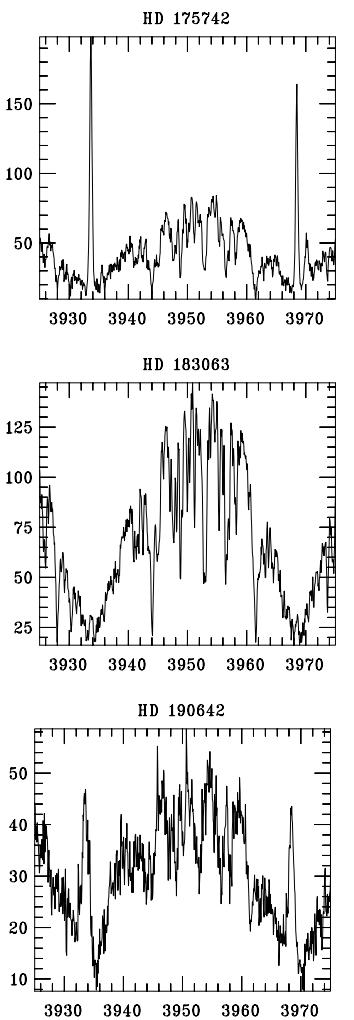

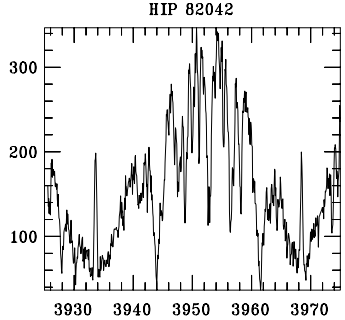

HD 155802
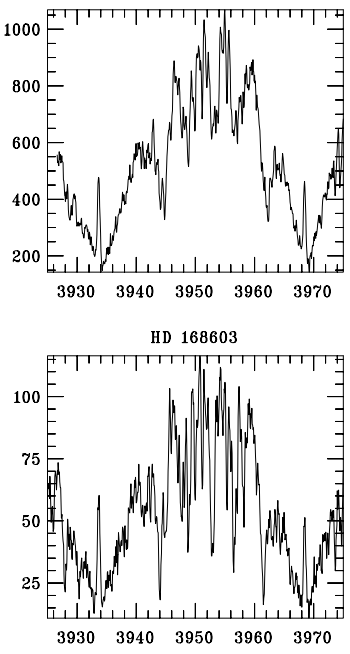

HD 176157
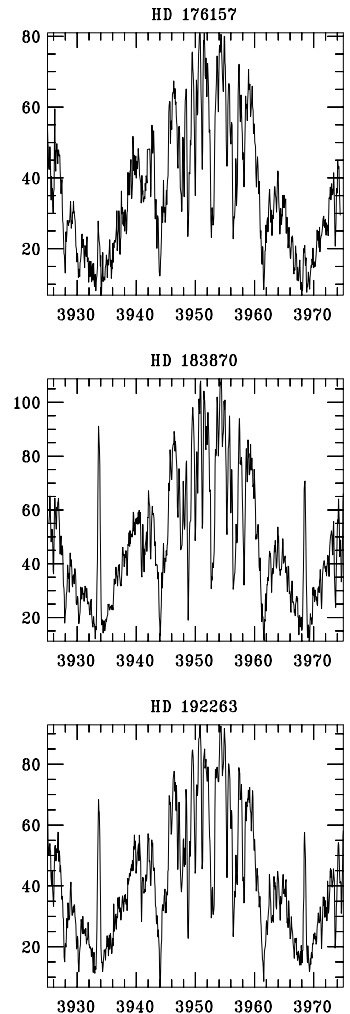

Fig. B1. continued 

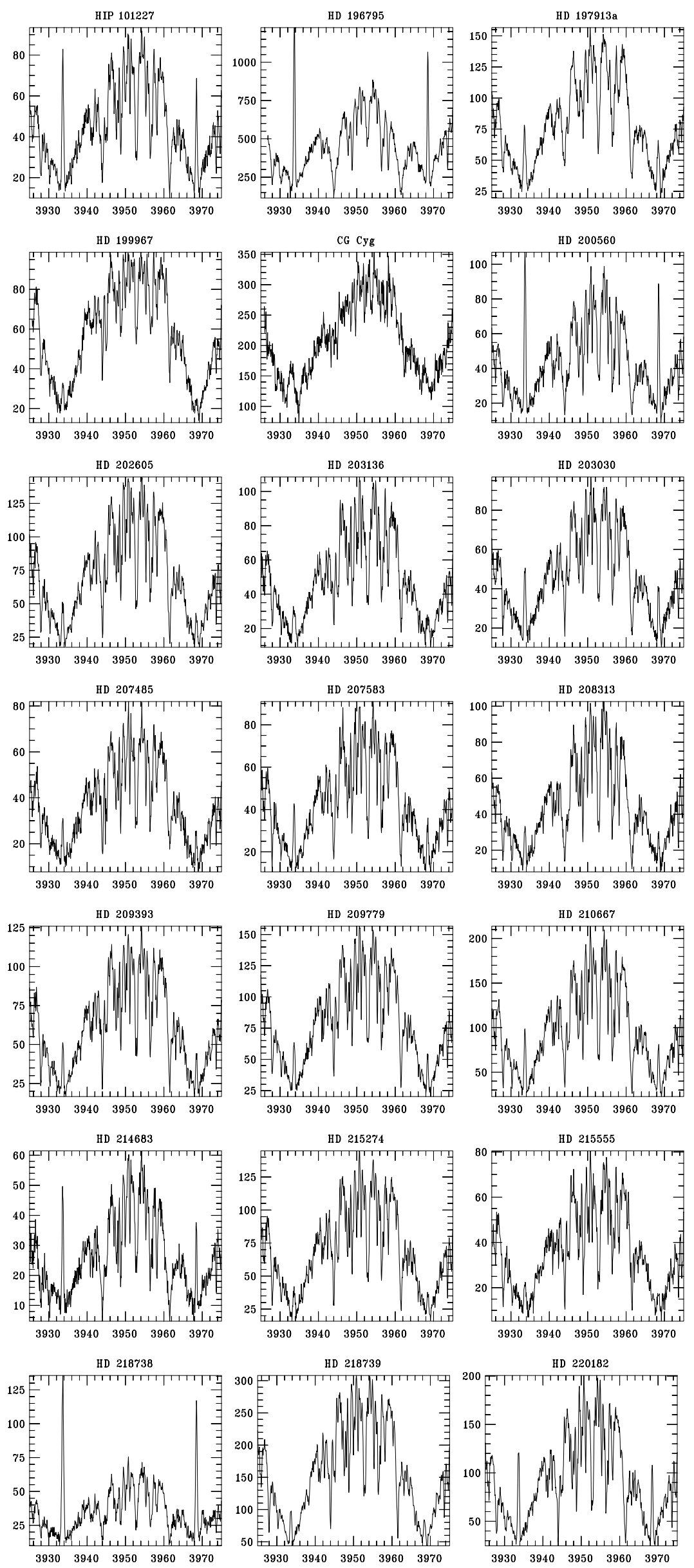

Fig. B1. continued
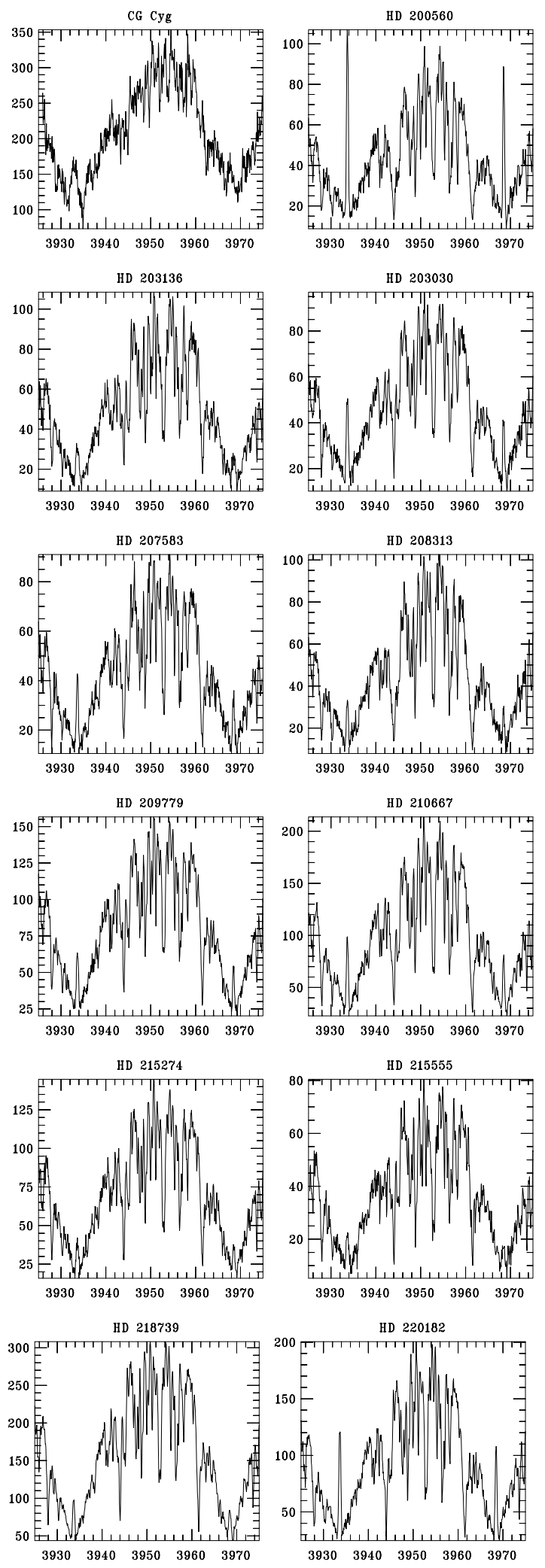
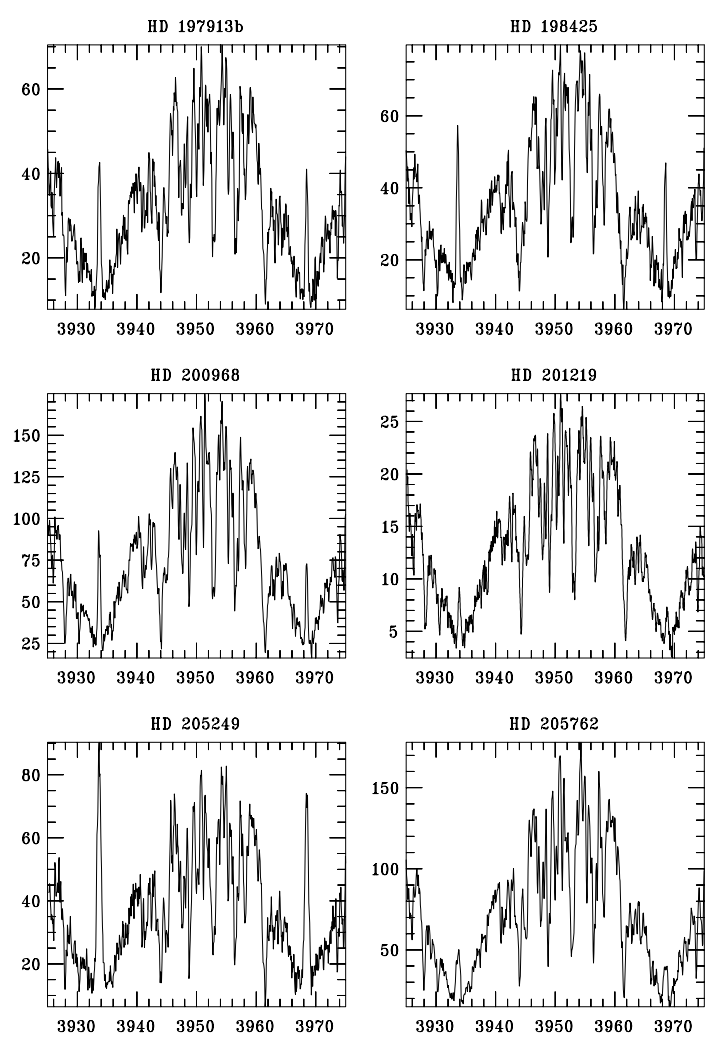

HD 208472
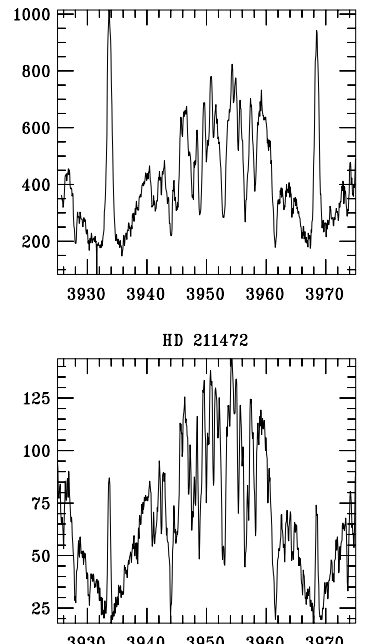
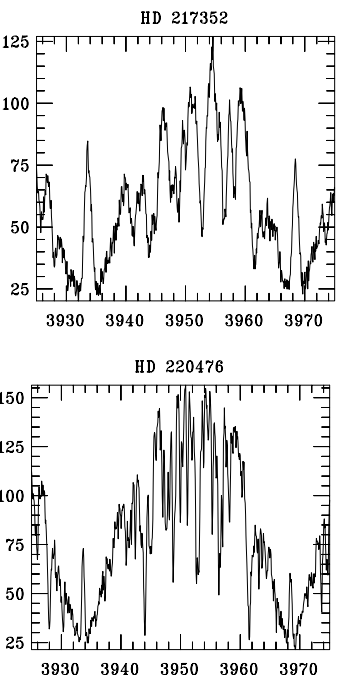
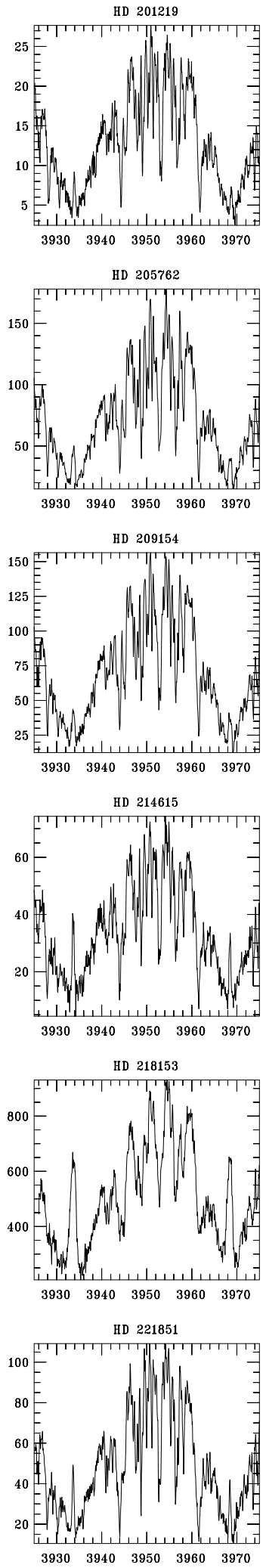
K.G. Strassmeier et al.: The Vienna-KPNO search for Doppler-imaging candidate stars. I.

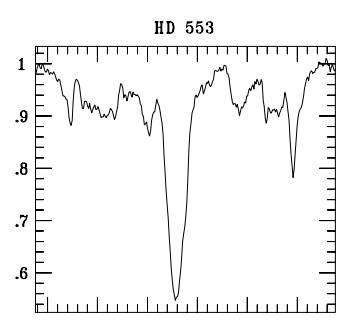

655065556560656565706575

HD 18632

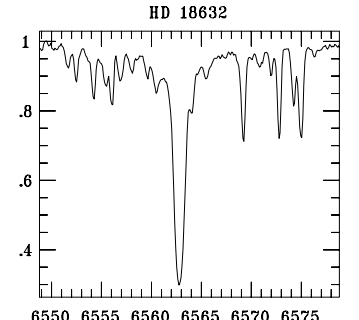

655065556560656565706575

SA0 150676

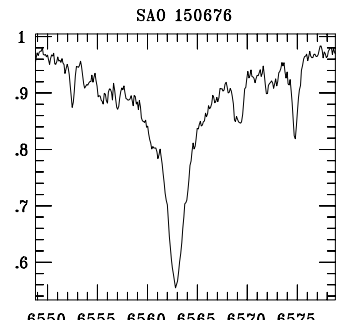

$6550 \quad 6555 \quad 6560 \quad 6565 \quad 6570 \quad 6575$

HD 261557

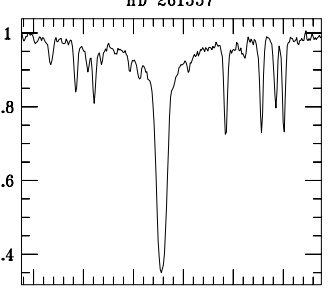

$655065556560 \quad 656565706575$

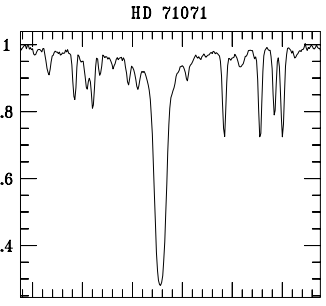

655065556560656565706575

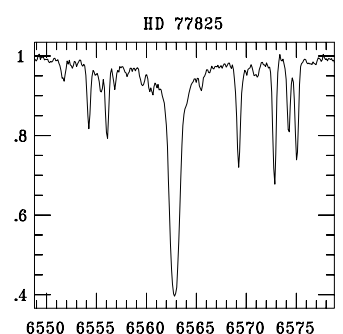

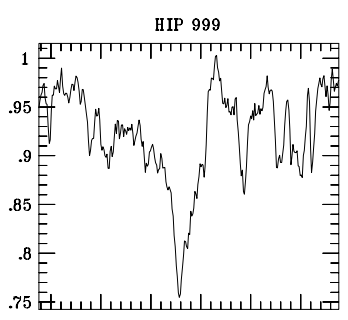

655065556560656565706575

HD 21845

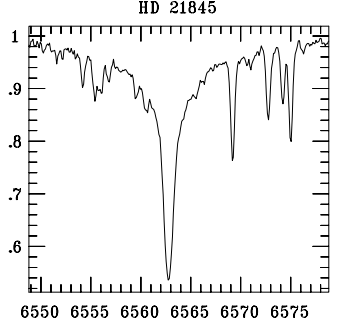

655065556560656565706575

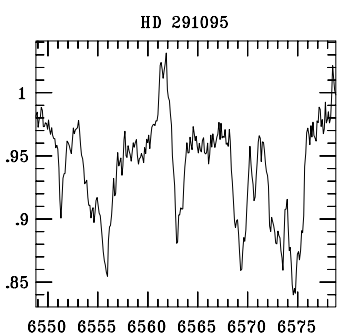

HD 56168

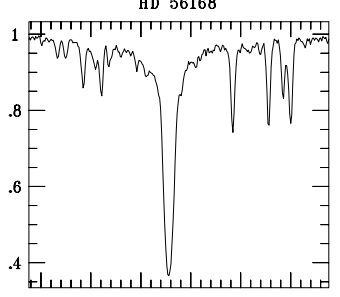

655065556560656565706575

HD 72146

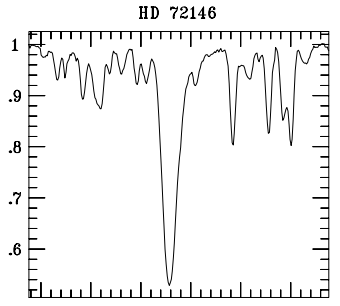

655065556560656565706575

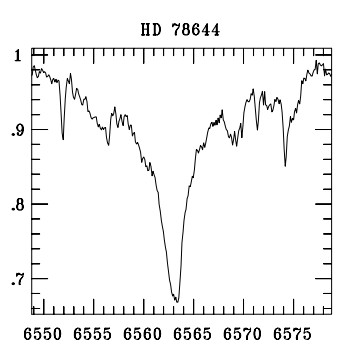

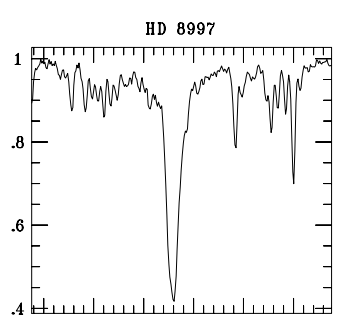

$6550 \quad 6555 \quad 6560 \quad 6565 \quad 6570 \quad 6575$

HD 23386

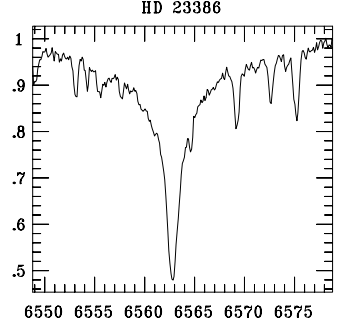

655065556560656565706575

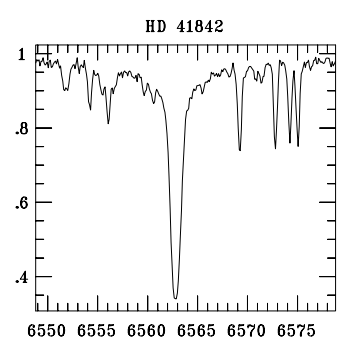

HD 60491

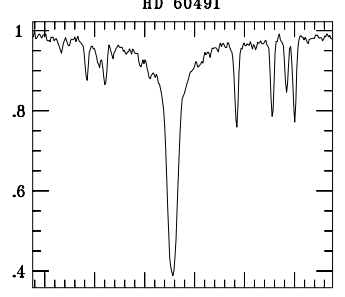

655065556560656565706575

HIP 42253

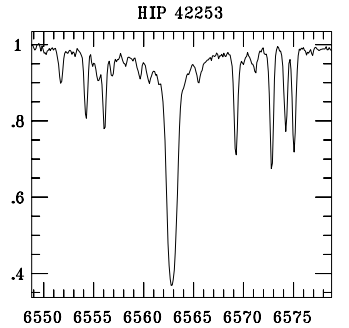

HIP 46634

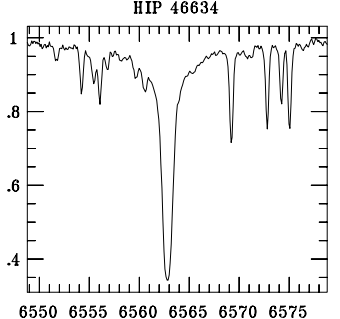

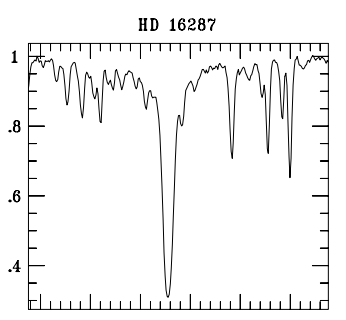

$65506555656065656570 \quad 6575$

HD 26900

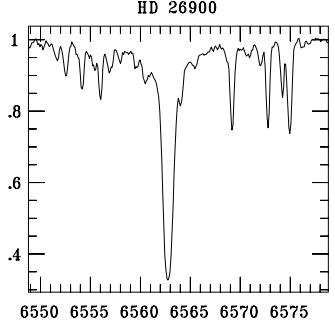

655065556560656565706575

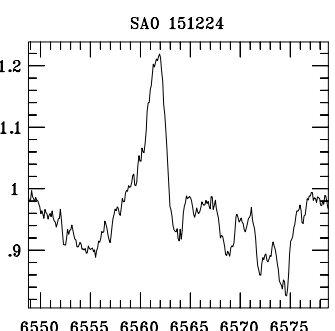

HD 62668

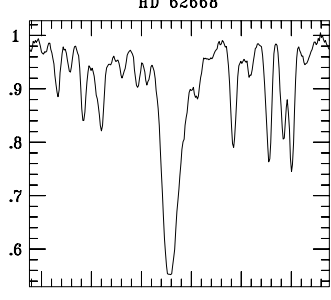

$655065556560 \quad 6565 \quad 6570 \quad 6575$

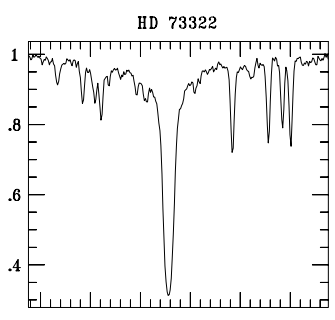

655065556560656565706575

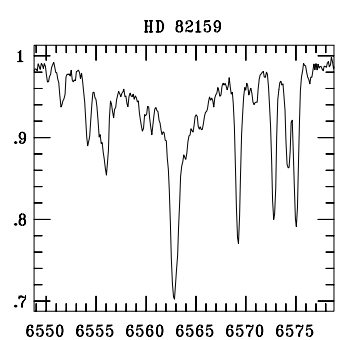

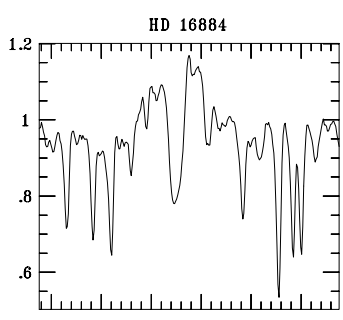

$6550 \quad 6555 \quad 6560 \quad 6565 \quad 6570 \quad 6575$

HD 28495

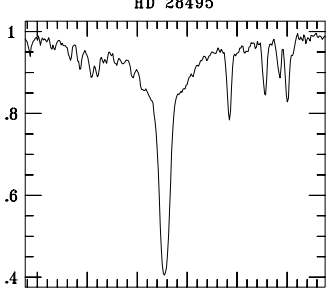

655065556560656565706575

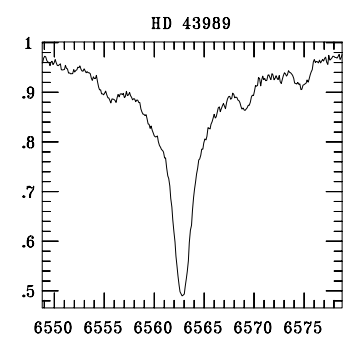

HIP 40774

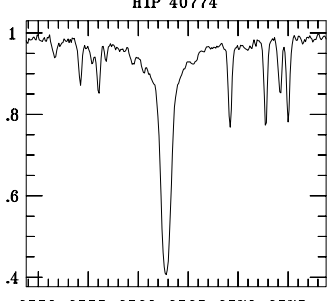

$6550 \quad 65556560 \quad 6565 \quad 65706575$

HIP 43422

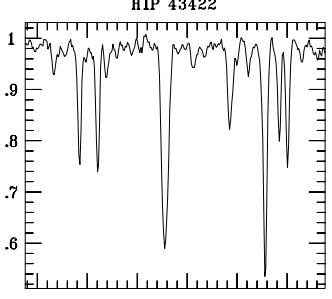

655065556560656565706575

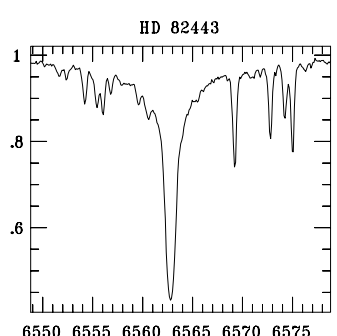

Fig. B2. Selected Balmer $\mathrm{H} \alpha$ spectra of stars with strong $\mathrm{Ca}$ II $\mathrm{H}$ and $\mathrm{K}$ emission. The HD number (or HIP number if no HD number exists) is identified on top of each plot. The wavelength range shown is $30 \AA$ 


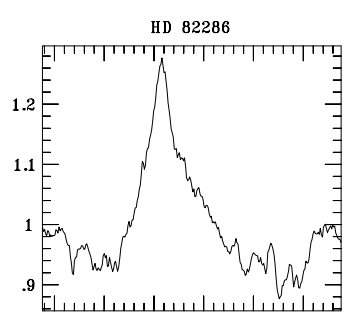

$6550 \quad 65556560 \quad 6565 \quad 6570 \quad 6575$
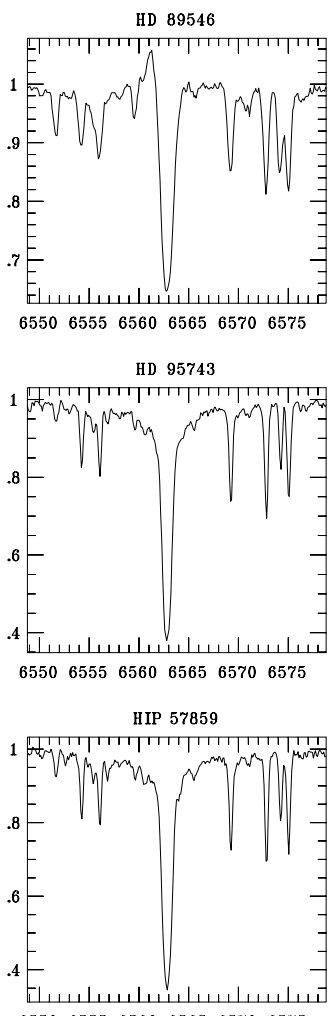

$655065556560 \quad 6565 \quad 6570 \quad 6575$

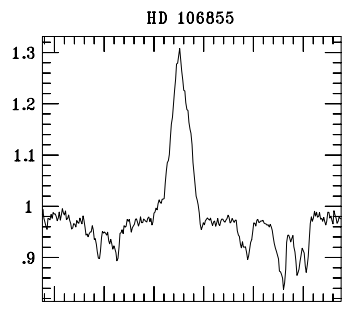

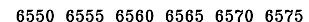

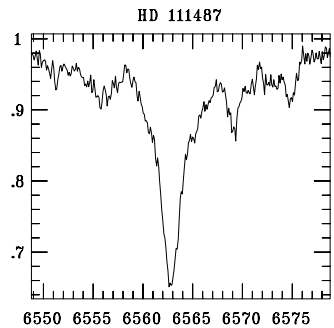

IP 63442

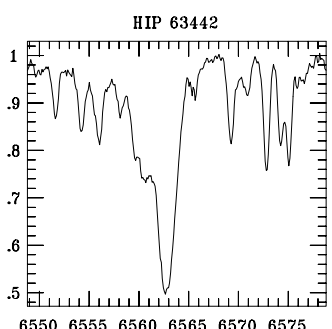

Fig. B2. continued

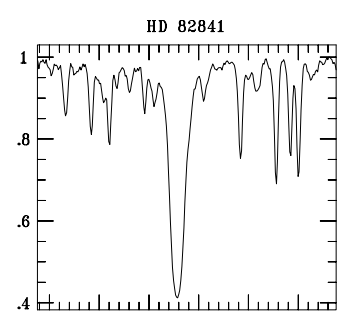

$65506555 \quad 6560 \quad 6565 \quad 6570 \quad 6575$

HD 92945

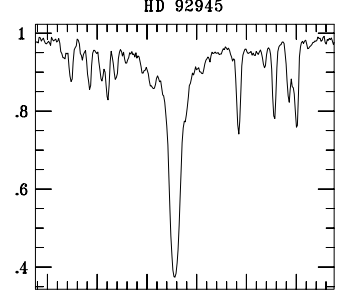

655065556560656565706575

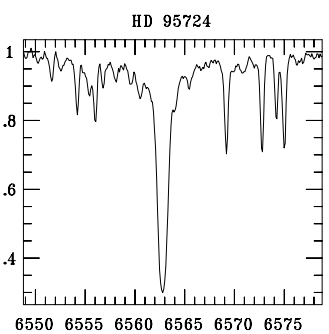

$$
\text { HD } 103720
$$

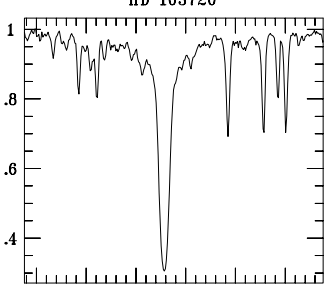

655065556560656565706575

HD 109011

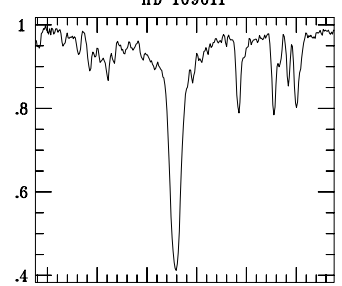

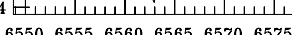

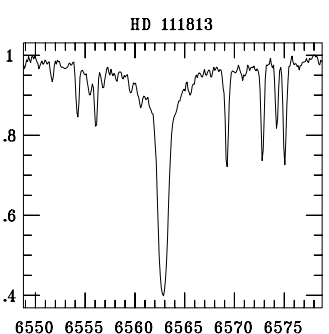

HD $11324 \%$

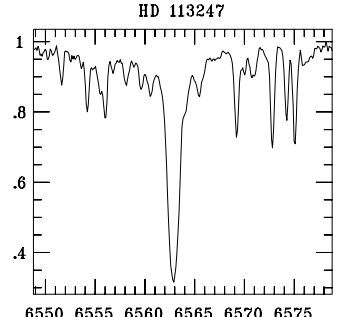

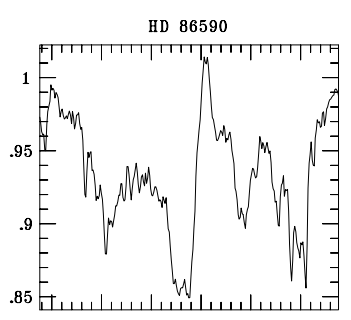

$6550655565606565 \quad 6570 \quad 6575$

HD 93811

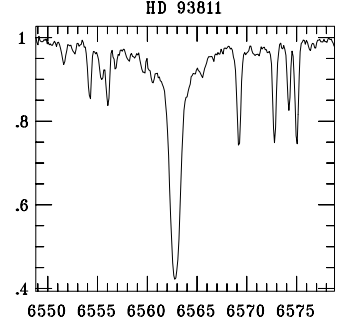

HD 97305

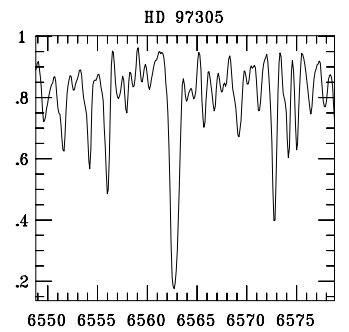

HD 105575

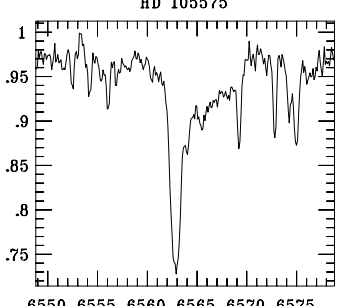

655065556560656565706575

HD 109647

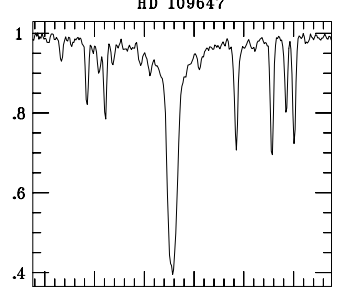

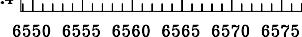

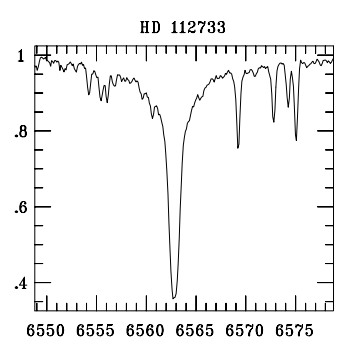

655065556560656565706575

HD 113449

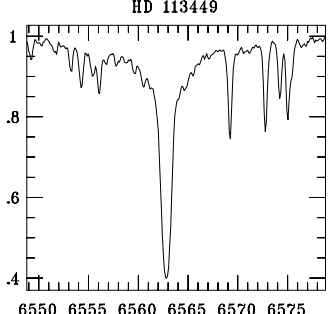

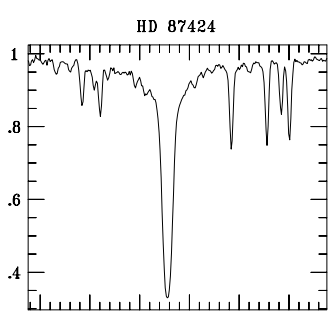

$65506555656065656570 \quad 6575$

HD 237944

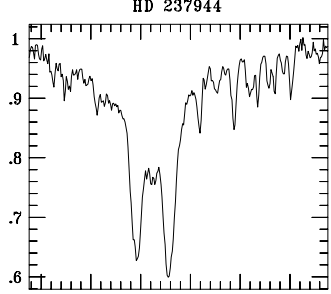

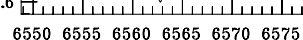

HD 101206

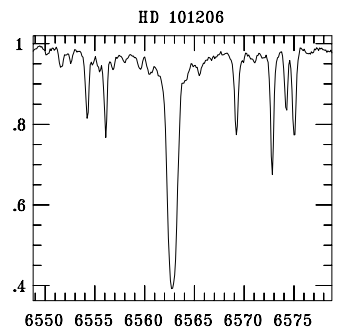

HD 105963

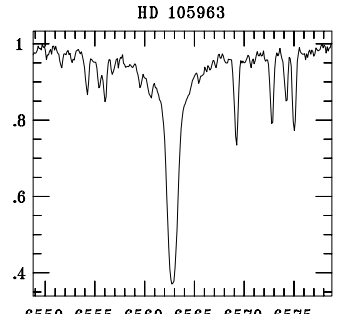

655065556560656565706575

HD 109703

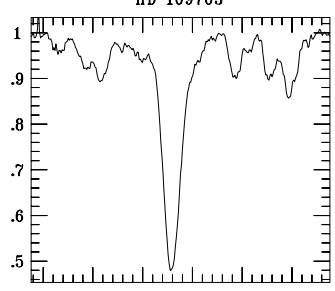

655065556560656565706575

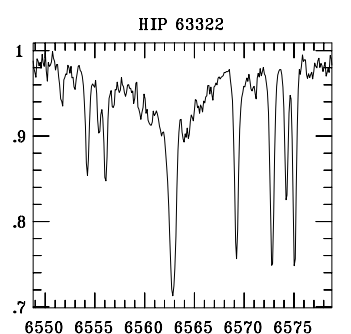

.655065556560656565706575

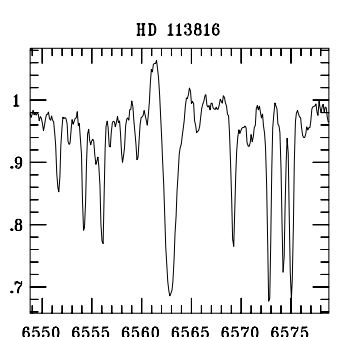

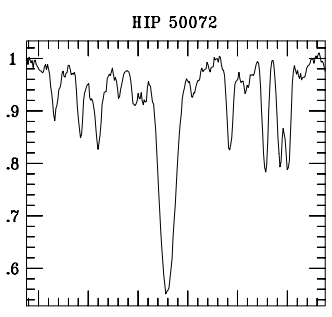

$6550 \quad 6555 \quad 6560 \quad 6565 \quad 6570 \quad 6575$

HD 95559

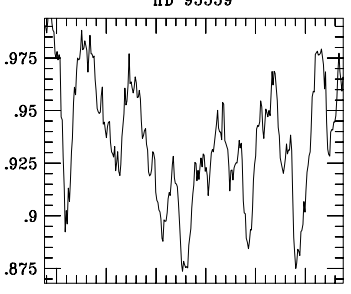

655065556560656565706575
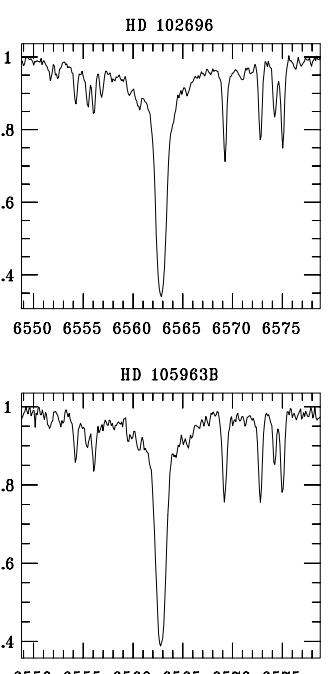

$655065556560 \quad 6565 \quad 65706575$

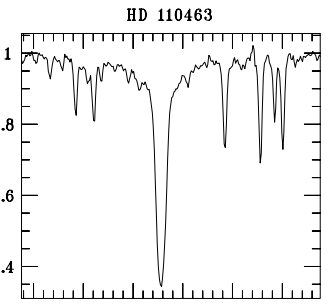

$6550 \quad 6555 \quad 6560 \quad 6565 \quad 6570 \quad 6575$

HD 112859

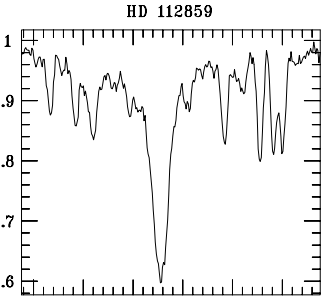
$655065556560 \quad 656565706575$

HD 116544

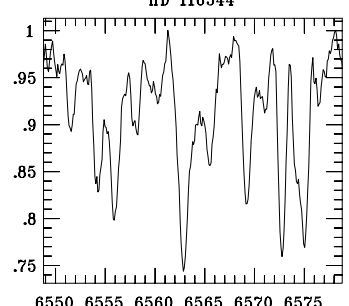




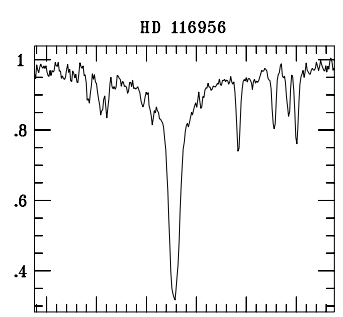

655065556560656565706575

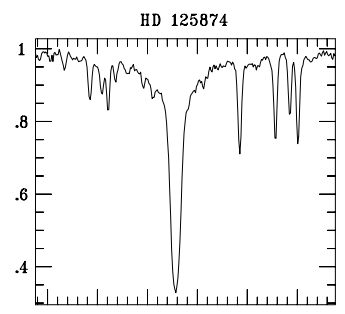

655065556560656565706575

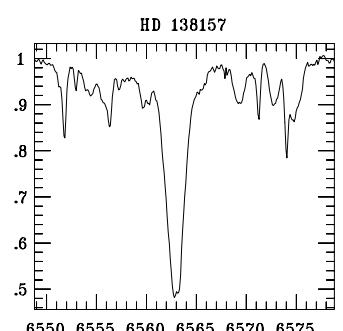

HD 147866

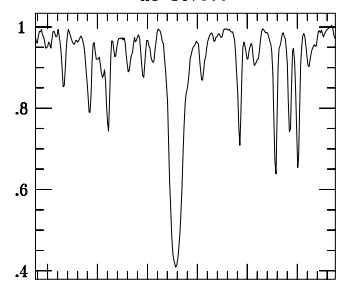

655065556560656565706575

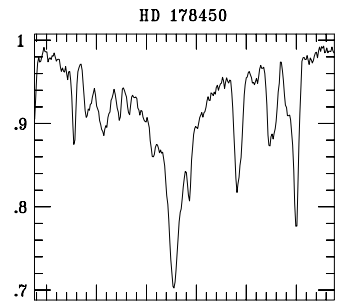

655065556560656565706575

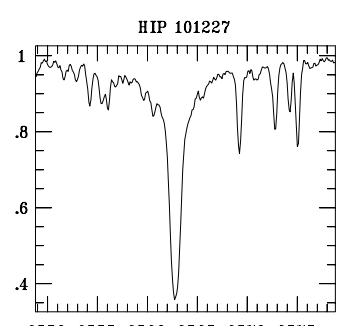

655065556560656565706575

HD 208472

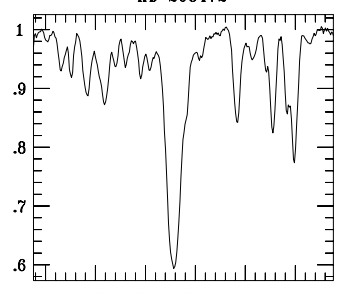

655065556560656565706575

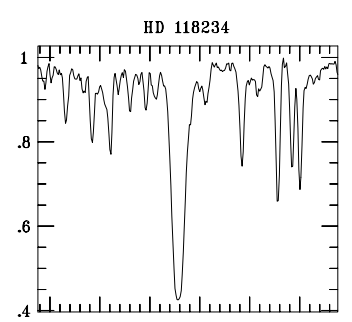

655065556560656565706575

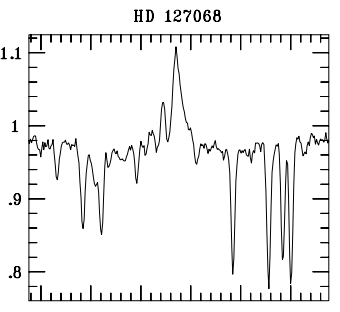

655065556560656565706575

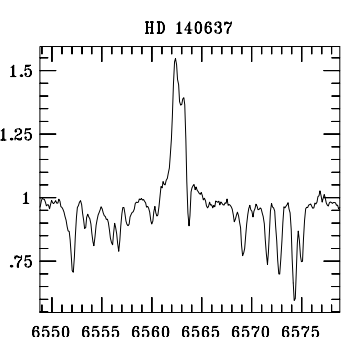

HD 152178

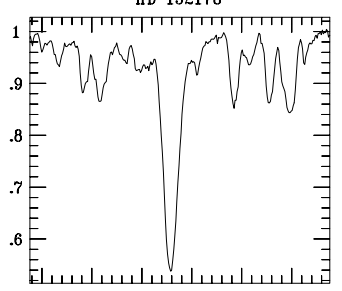

$655065556560 \quad 6565 \quad 65706575$

HD 180642

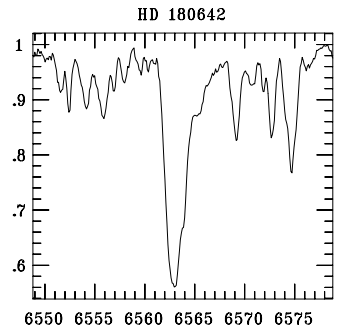

655065556560656565706575

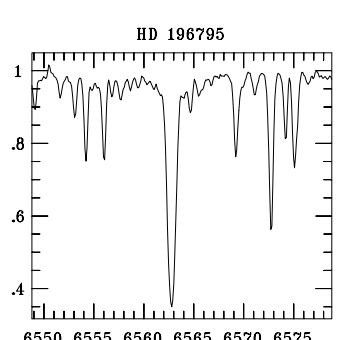

HD 214683

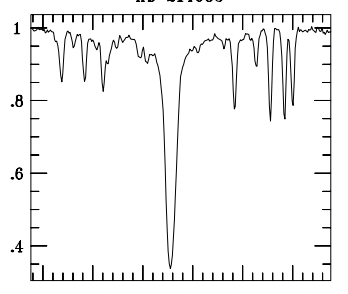

655065556560656565706575

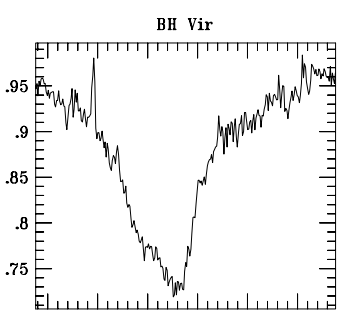

655065556560656565706575

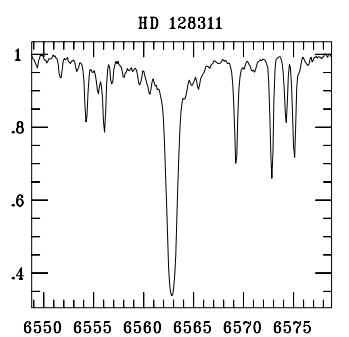

HD 142680

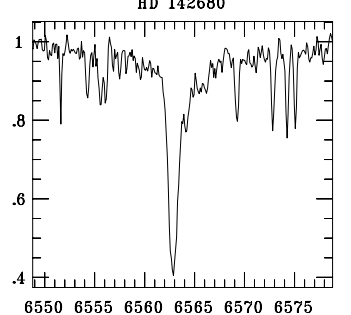

HD 153557

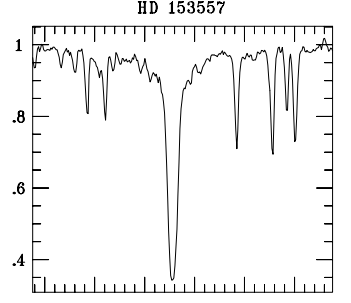

$655065556560 \quad 656565706575$

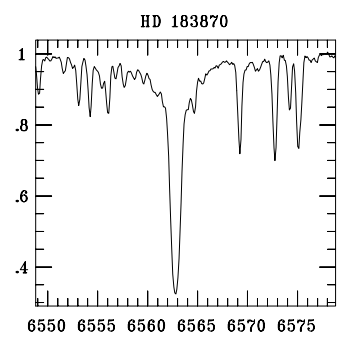

HD 198425

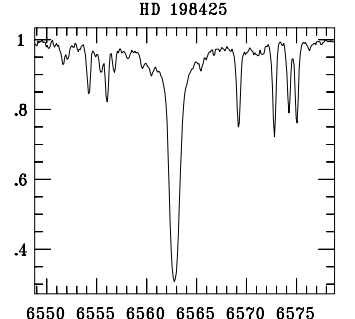

HD 217352

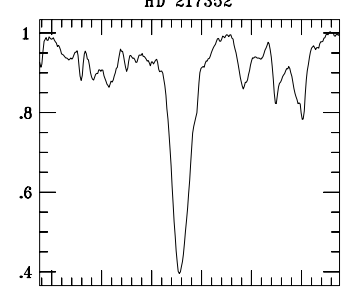

655065556560656565706575

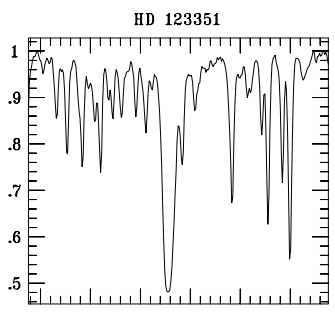

$6550 \quad 65556560 \quad 6565 \quad 6570 \quad 6575$

HD 131977

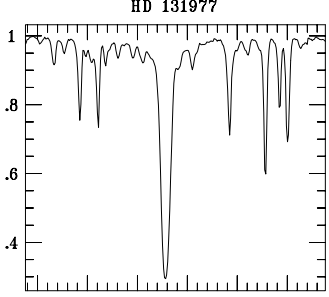

655065556560656565706575

HD 143937

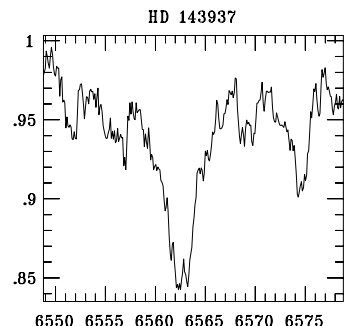

IID 163621

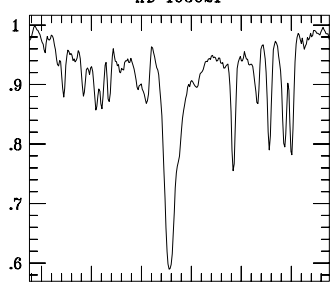

$6550 \quad 65556560656565706575$

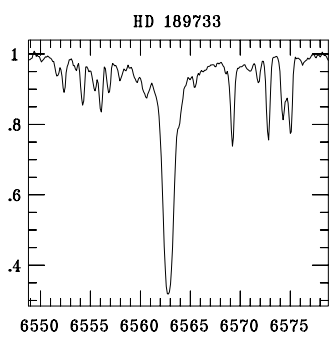

CG Cyg

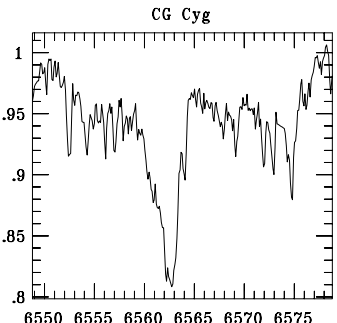

HD 218153

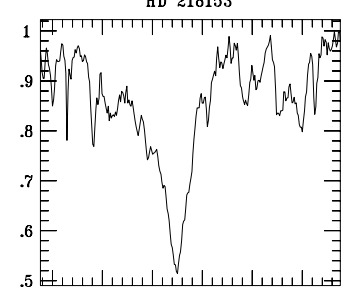

655065556560656565706575

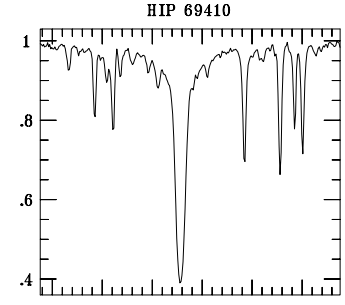

$6550 \quad 6555 \quad 6560 \quad 6565 \quad 6570 \quad 6575$

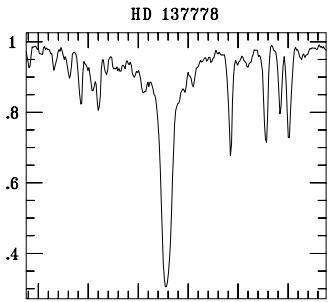

655065556560656565706575

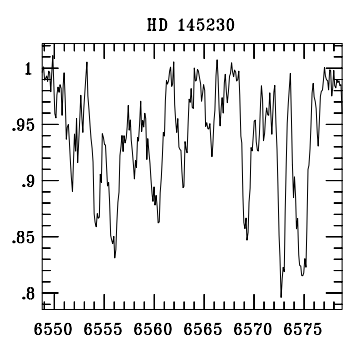

HD 171488

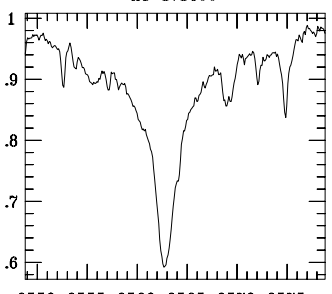

$6550 \quad 6555 \quad 6560 \quad 6565 \quad 6570 \quad 6575$

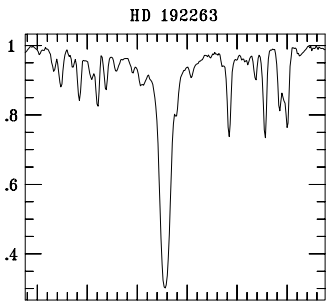

$655065556560 \quad 6565 \quad 65706575$

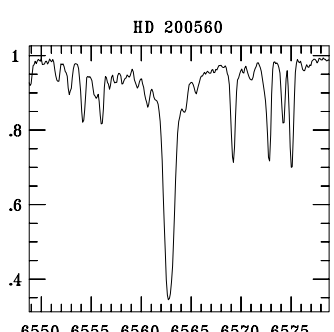

$6550 \quad 65556560656565706575$

HD 218738

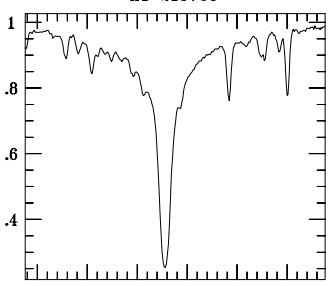

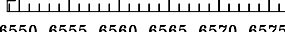

Fig. B2. continued 

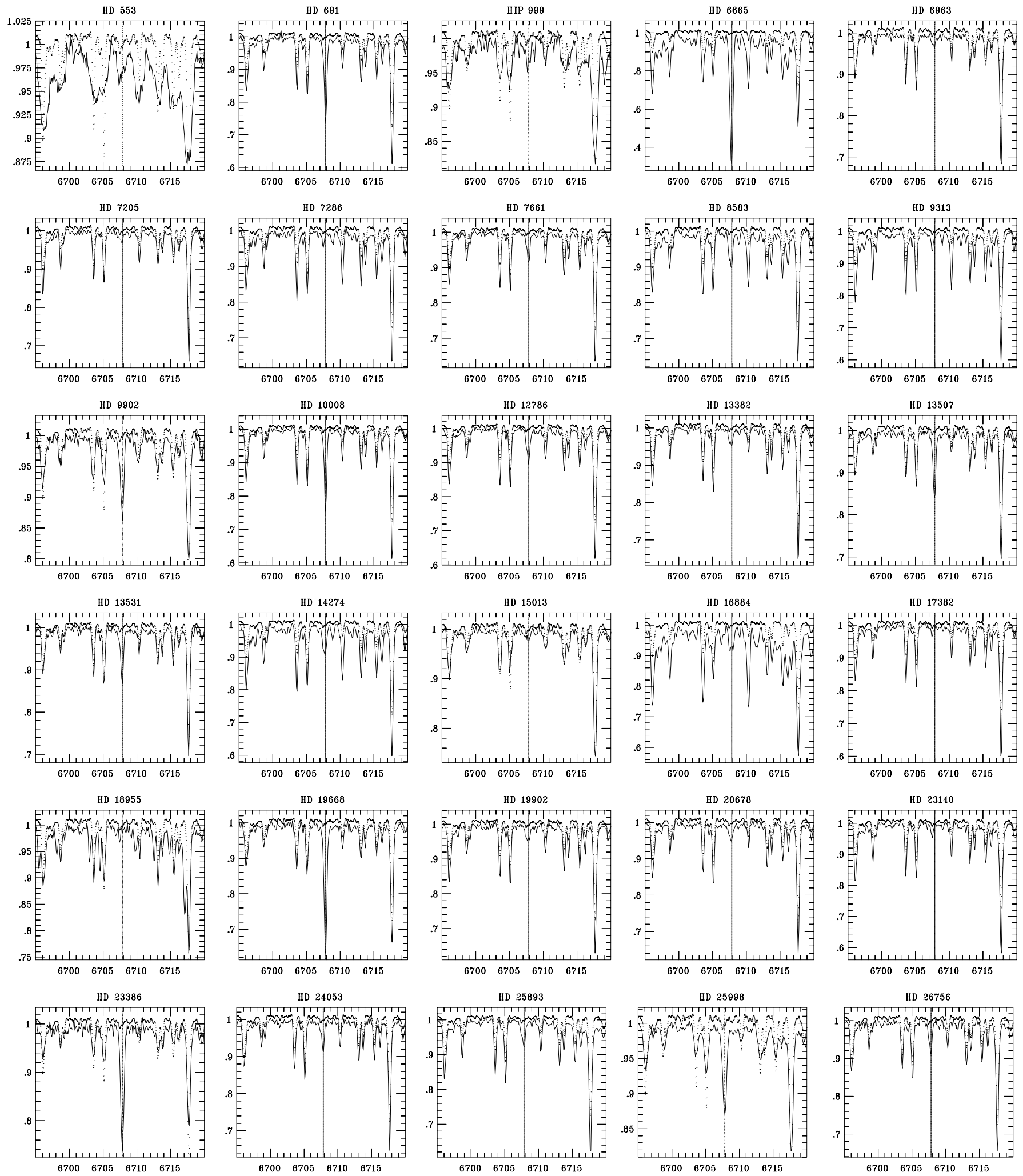

Fig. B3. Lithium 6707.8- $\AA$ spectra of stars with Ca II H and $\mathrm{K}$ emission and $W_{\mathrm{Li}} \geq 10 \mathrm{~m} \AA$. The HD number (or HIP number if no HD number exists) is identified on top of each plot. The wavelength range shown is $25 \AA$. The dotted spectrum is a spectrum of the Sun vertically shifted by 0.01 for comparison purposes (the solar lithium equivalent width is $\approx 2 \mathrm{~m} \AA$ ). The vertical line is the average rest wavelength of Li I at $6707.8 \AA$ 
K.G. Strassmeier et al.: The Vienna-KPNO search for Doppler-imaging candidate stars. I.
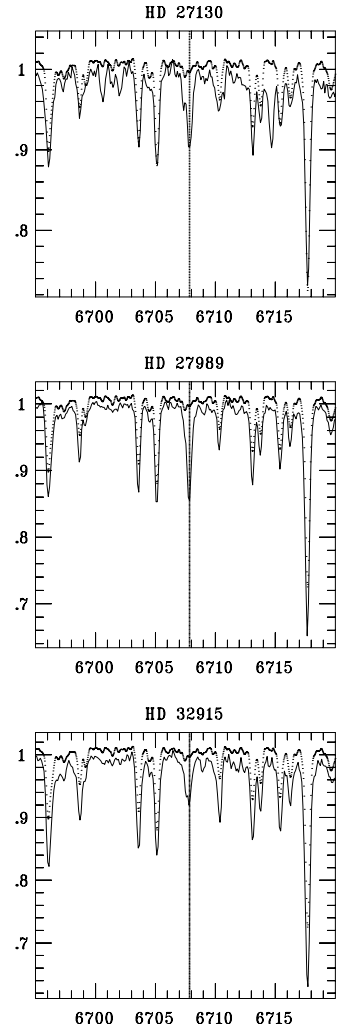

$6700 \quad 6705 \quad 6710 \quad 6715$

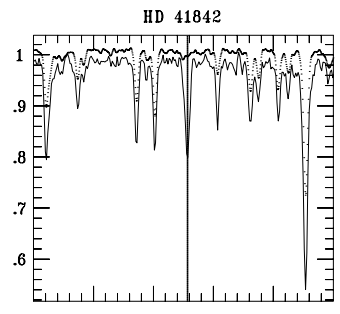

$6700 \quad 6705 \quad 6710 \quad 6715$

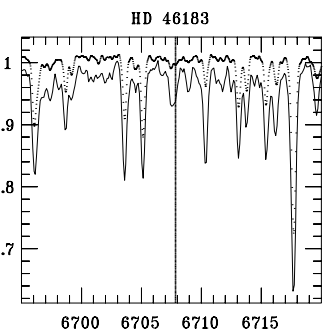

HD 56168

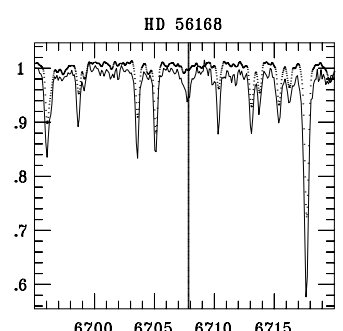

$6700 \quad 6705 \quad 6710 \quad 6715$

HD 64942

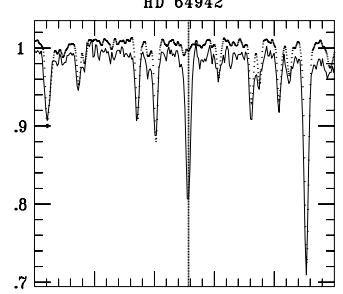

$6700 \quad 6705 \quad 6710 \quad 6715$
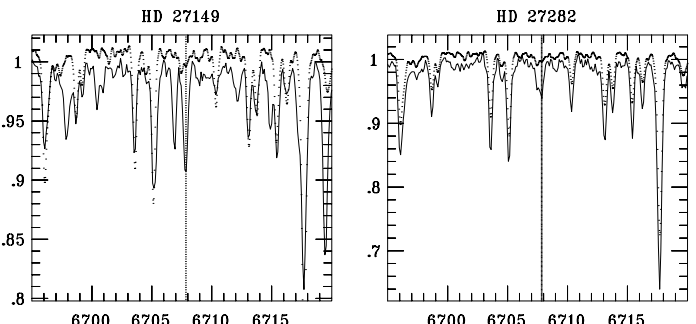

$\begin{array}{lllll}6700 & 6705 & 6710 & 6715\end{array}$
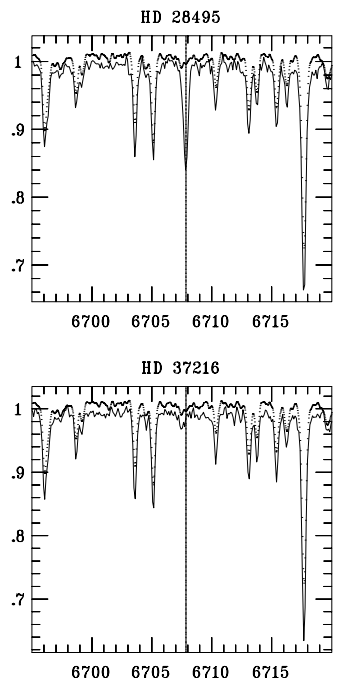

SA0 151224
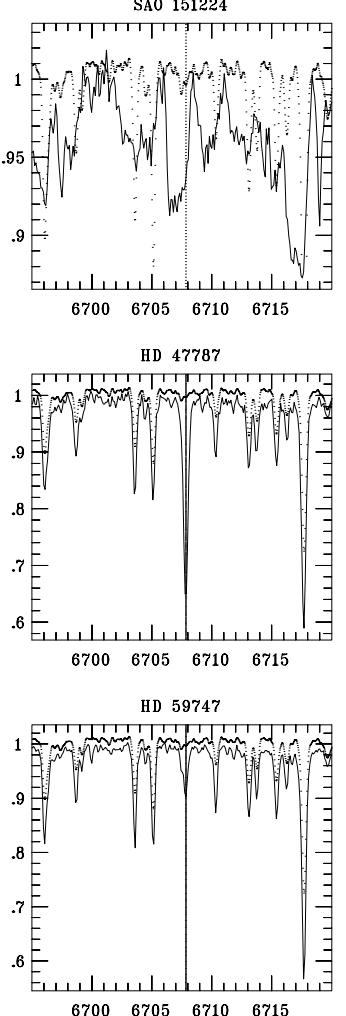

HD 64725

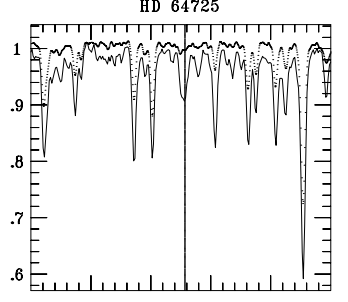

$6700 \quad 6705 \quad 6710 \quad 6715$
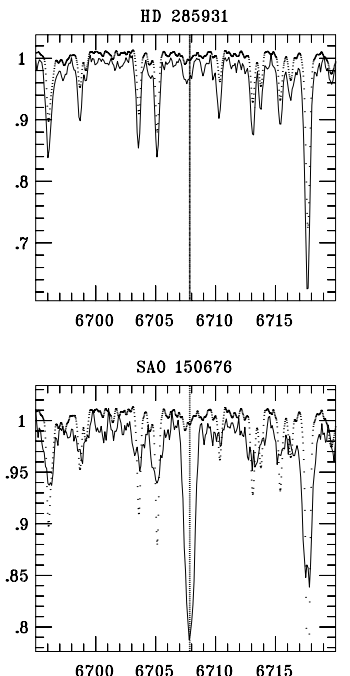

HD 43516
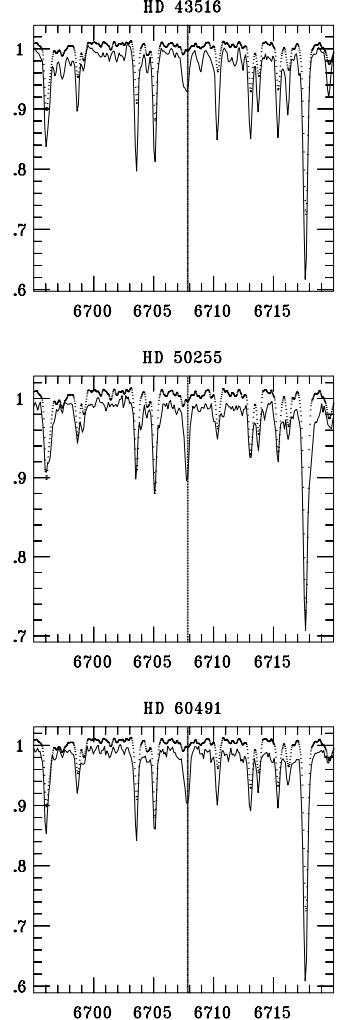

HIP 40774

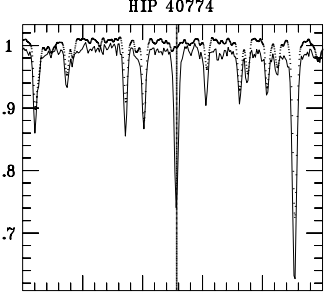

$\begin{array}{lllllll}6700 & 6705 & 6710 & 6715\end{array}$

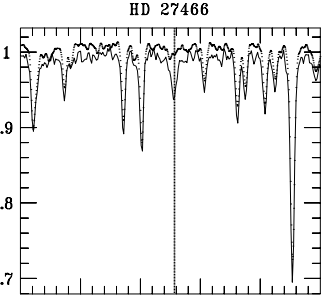

$\begin{array}{llll}6700 & 6705 & 6710 & 6715\end{array}$
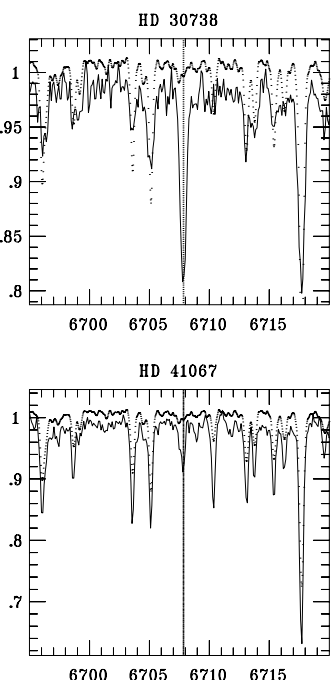

HD 43989
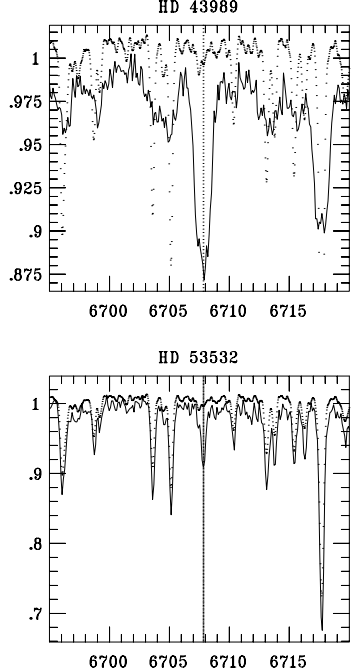

HD 62668

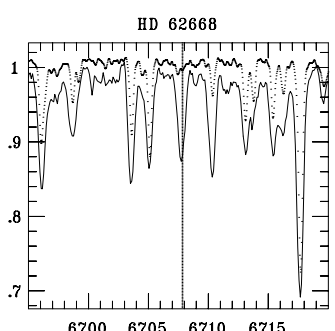

HD 69433

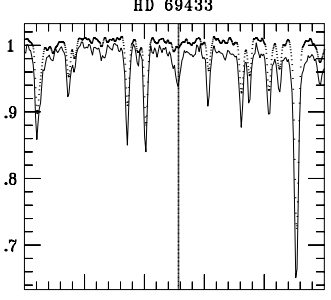

$\begin{array}{lllllll}6700 & 6705 & 6710 & 6715\end{array}$

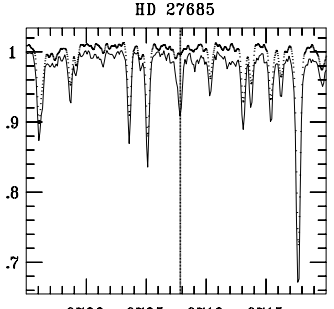

$6700 \quad 6705 \quad 6710 \quad 6715$

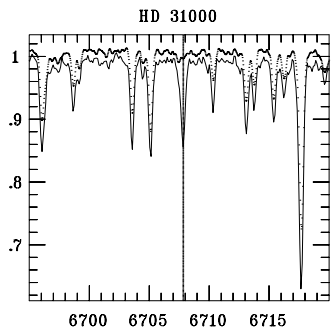

HD) 291095

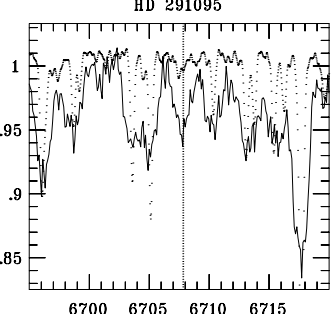

HD 45609
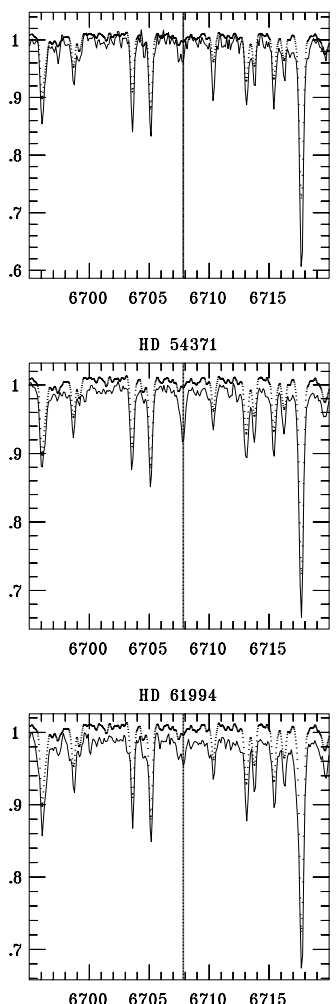

HD 70146

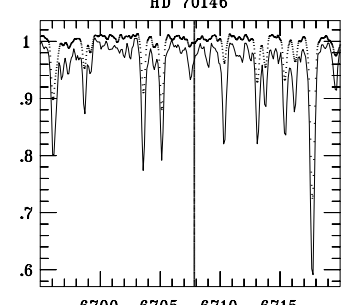

Fig. B3. continued 

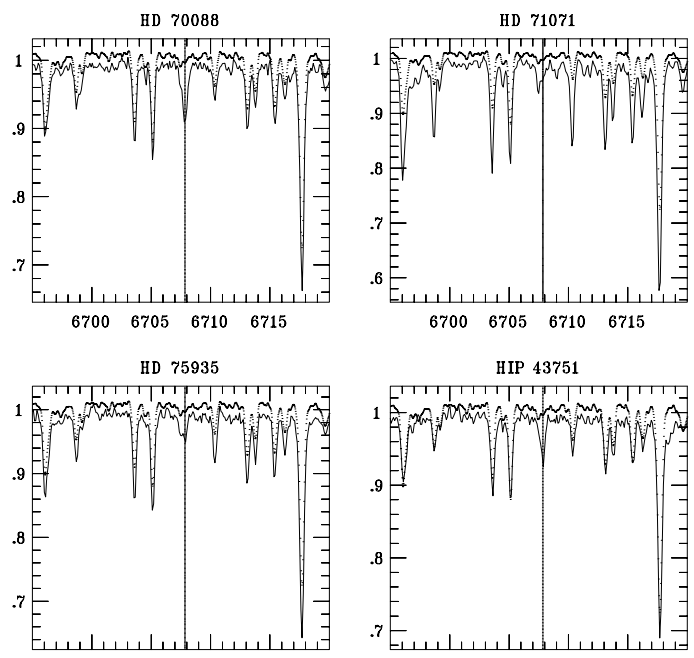

$\begin{array}{lllll}6700 & 6705 & 6710 & 6715\end{array}$

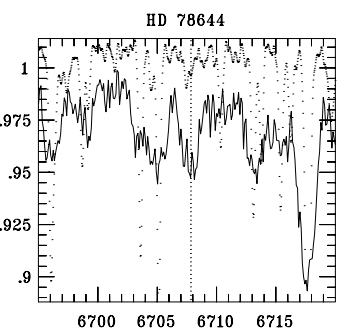

$\begin{array}{lllll}6700 & 6705 & 6710 & 6715\end{array}$
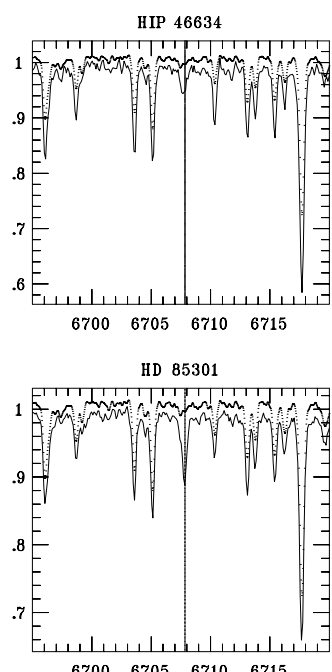

HD 92945

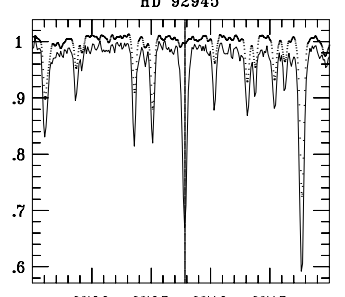

$\begin{array}{llll}6700 & 6705 & 6710 & 6715\end{array}$

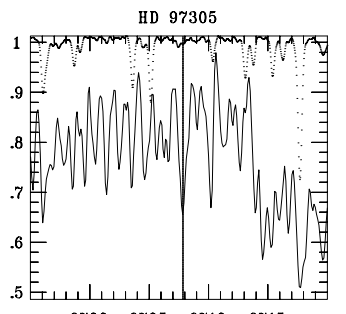

$\begin{array}{llllll}6700 & 6705 & 6710 & 6715\end{array}$

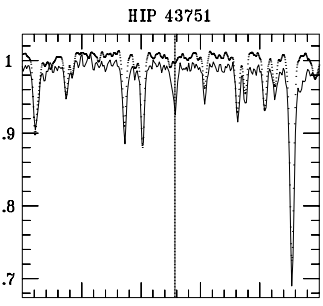

$\begin{array}{llll}6700 & 6705 & 6710 & 6715\end{array}$

HD 79993

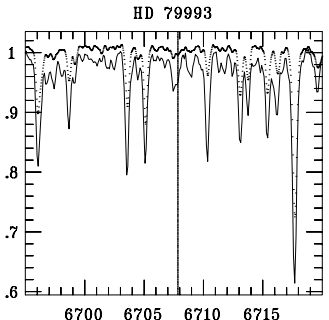

$\begin{array}{lllll}6700 & 6705 & 6710 & 6715\end{array}$
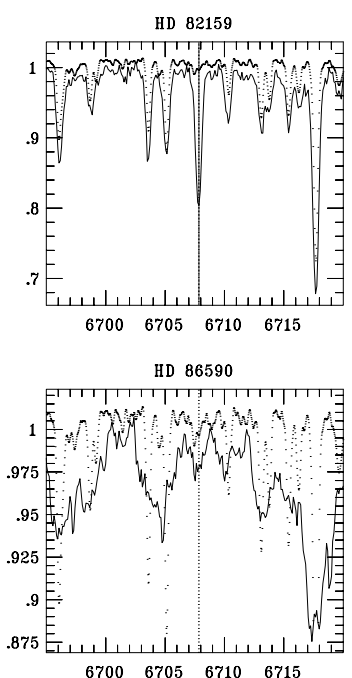

HD 93811

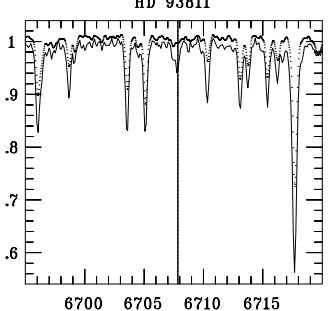

HD 97601

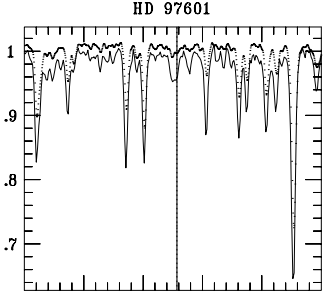

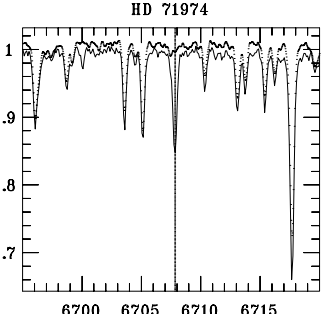

HD 76218

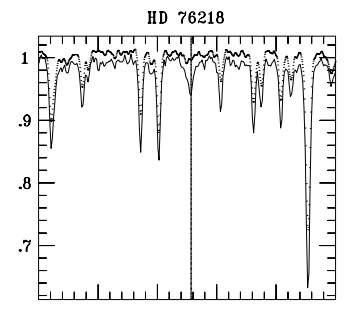

$\begin{array}{llll}6700 & 6705 & 6710 & 6715\end{array}$

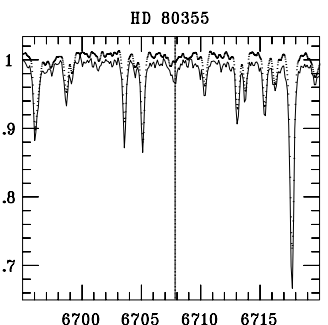

$\begin{array}{llllll}6700 & 6705 & 6710 & 6715\end{array}$
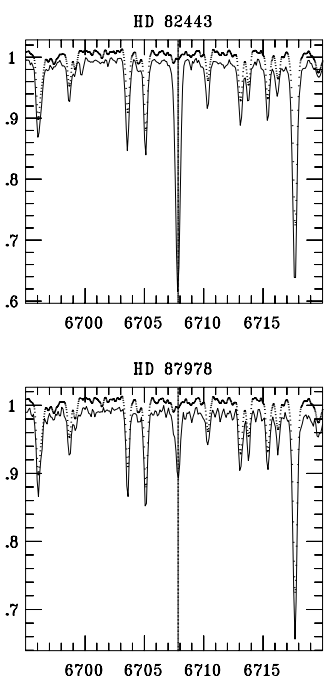

HD 93915

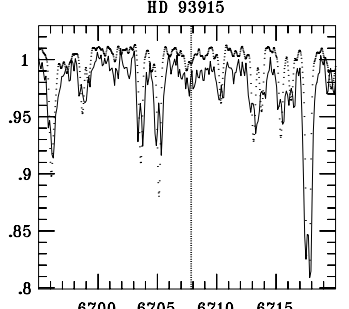

$\begin{array}{llll}6700 & 6705 & 6710 & 6715\end{array}$

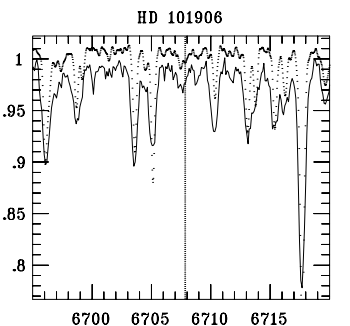

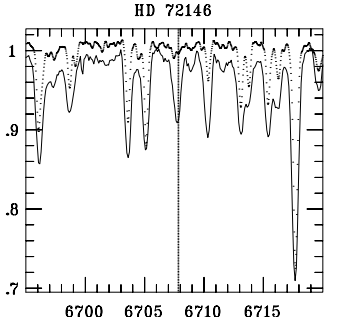

HD 76799

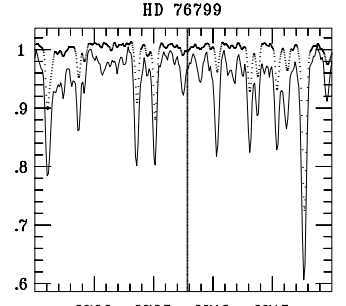

$\begin{array}{llll}6700 & 6705 & 6710 & 6715\end{array}$

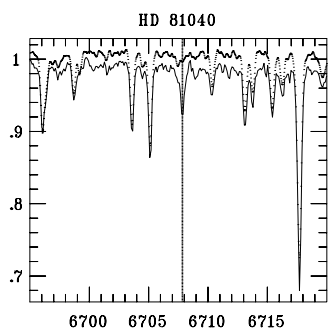

$6700 \quad 6705 \quad 6710 \quad 6715$

HD 82286
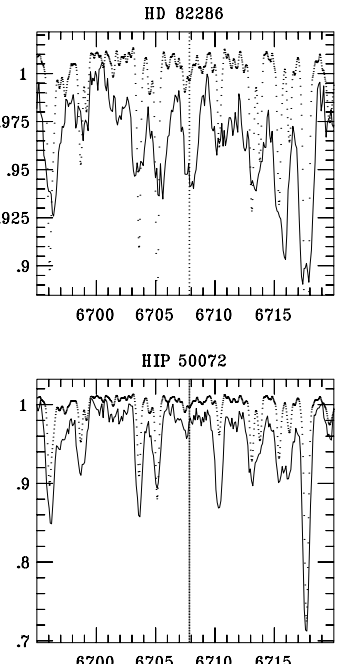

HD 95559
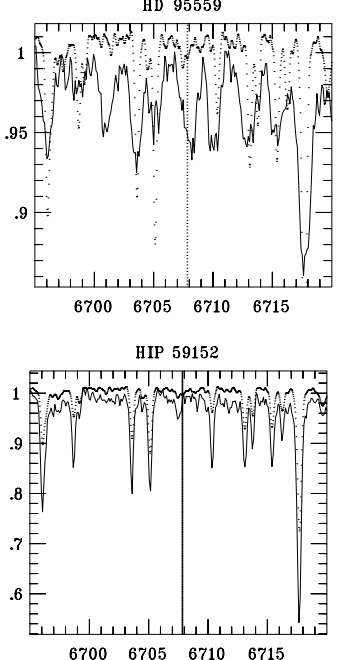

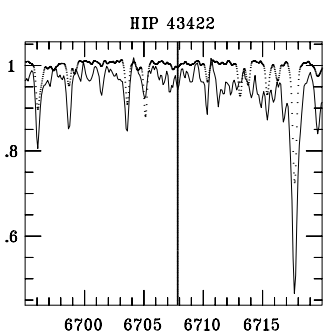

$\begin{array}{llll}6700 & 6705 & 6710 & 6715\end{array}$
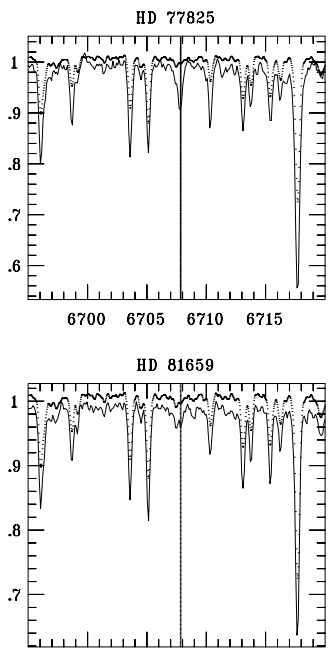

$\begin{array}{llllll}6700 & 6705 & 6710 & 6715\end{array}$
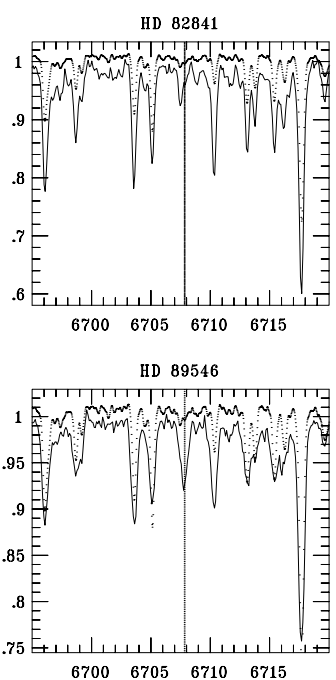

HD 96064

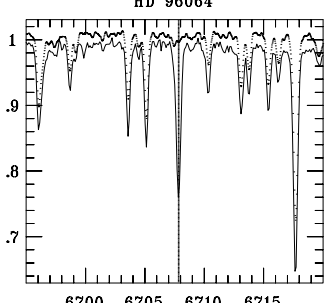

HD 105963

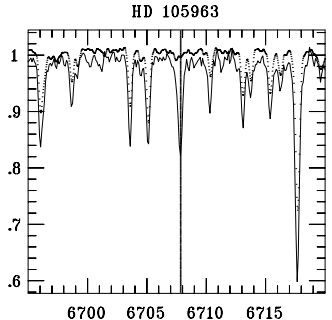

Fig. B3. continued 
K.G. Strassmeier et al.: The Vienna-KPNO search for Doppler-imaging candidate stars. I.
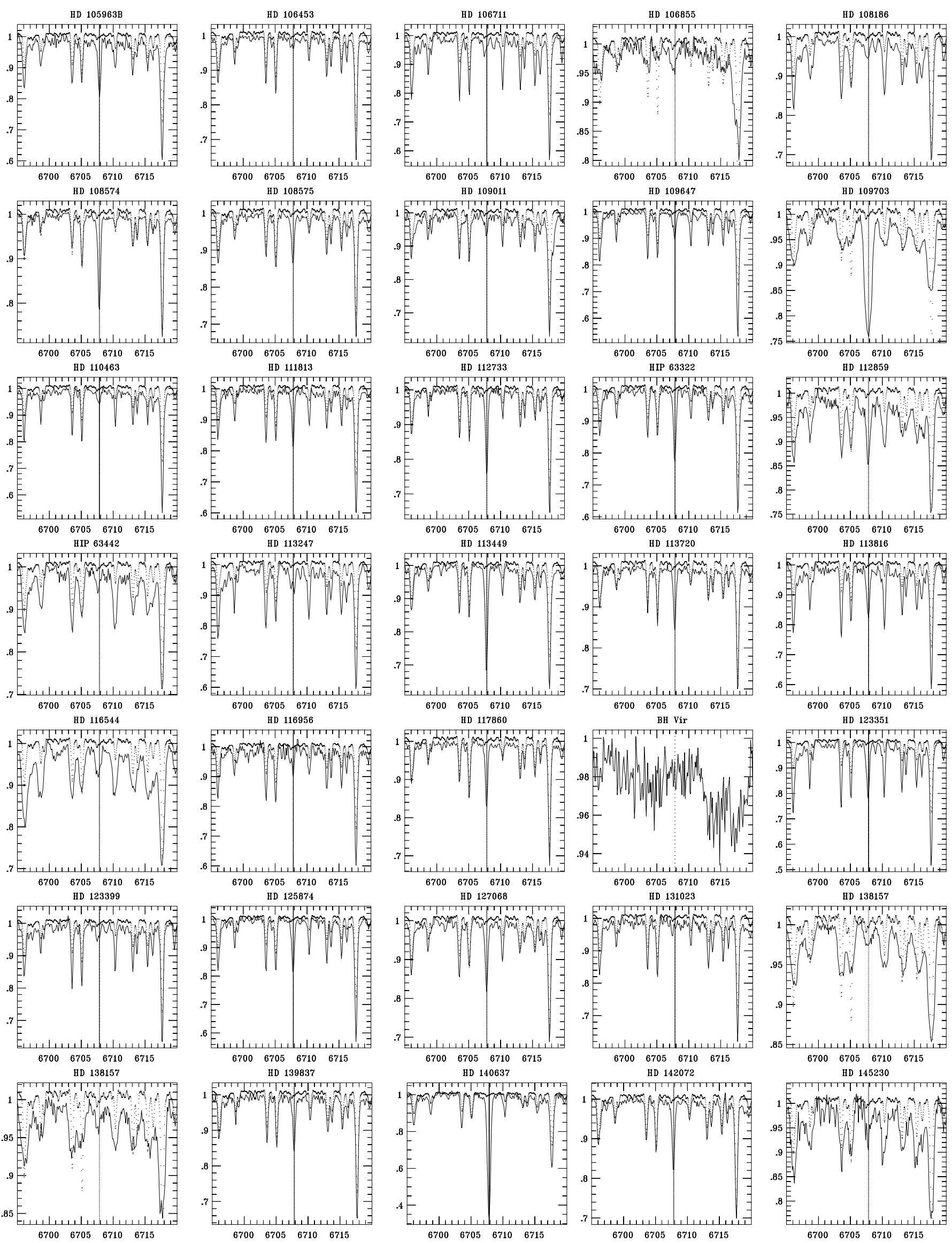

Fig. B3. continued 

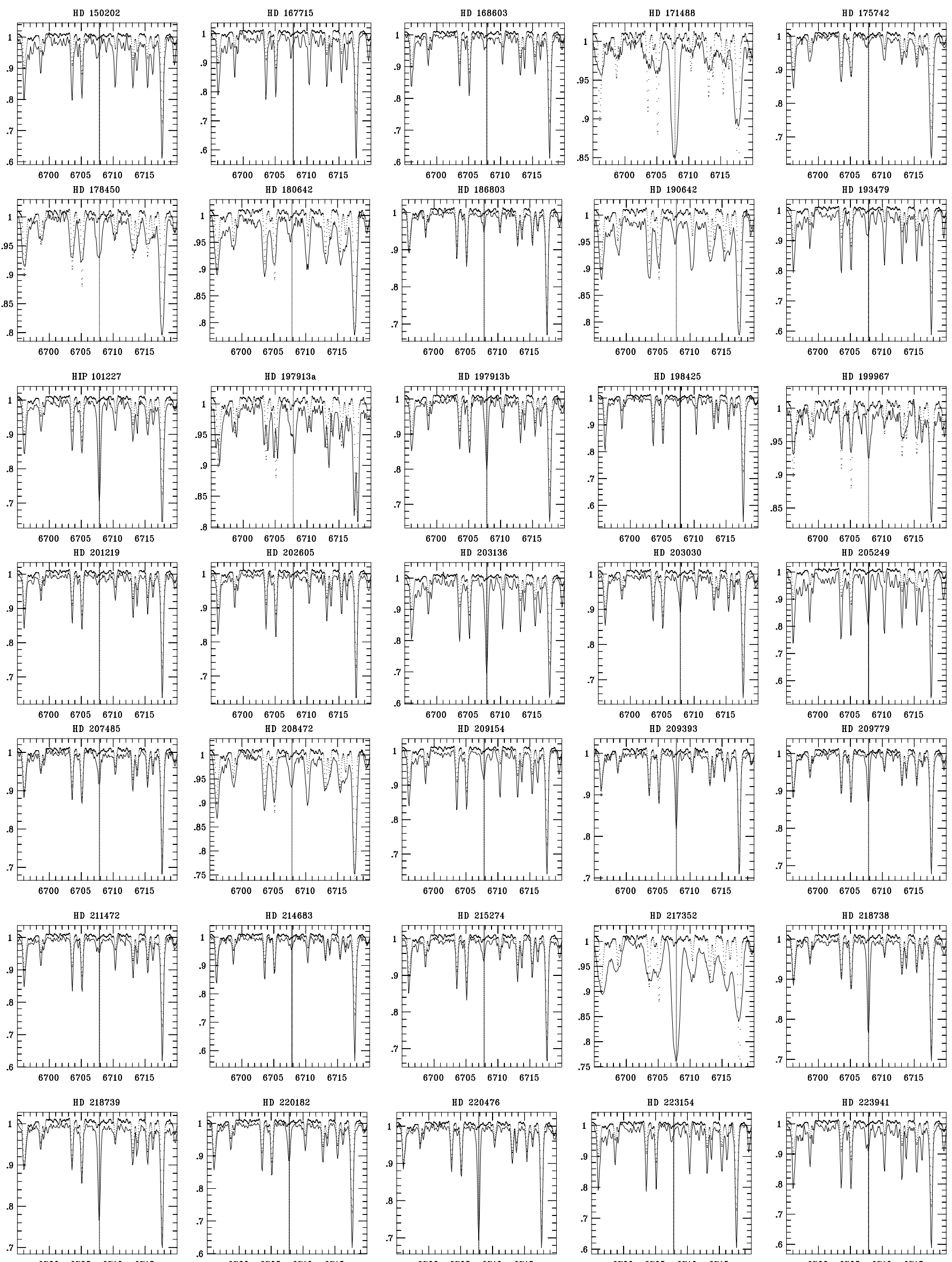

HD 220476

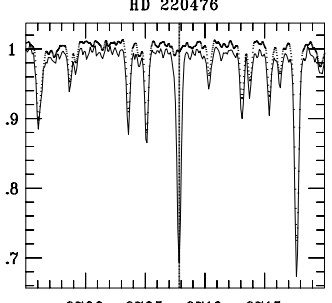

HD 223154

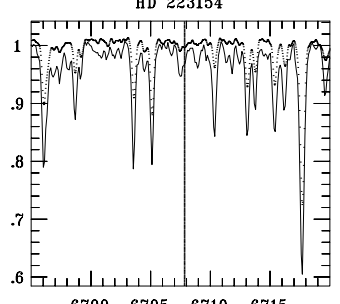

Fig. B3. continued
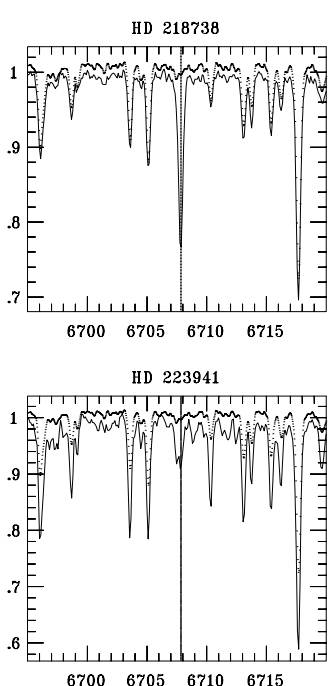

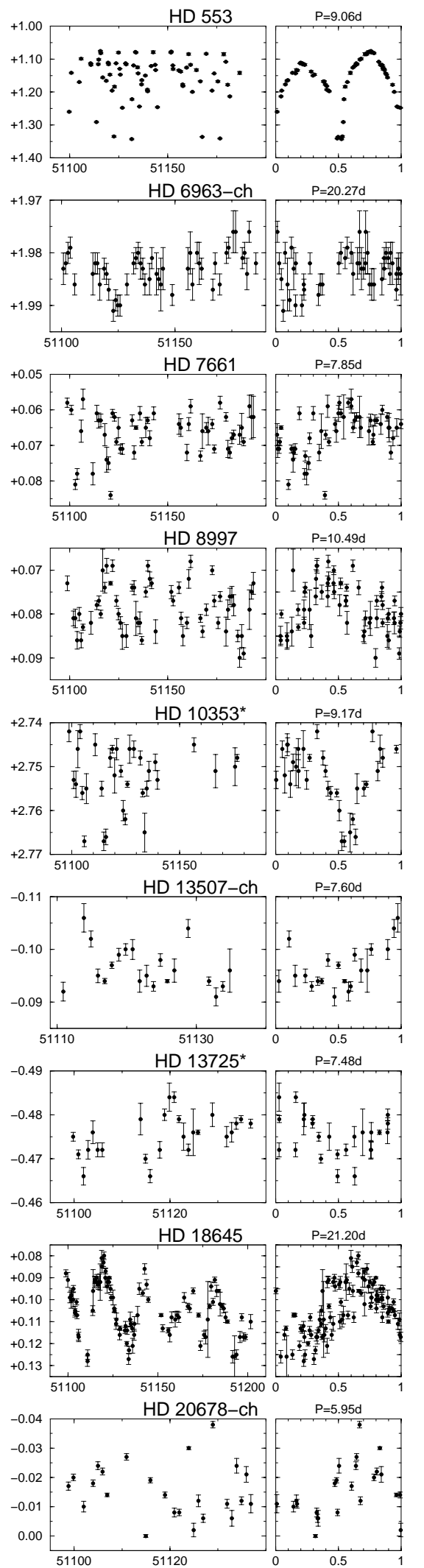
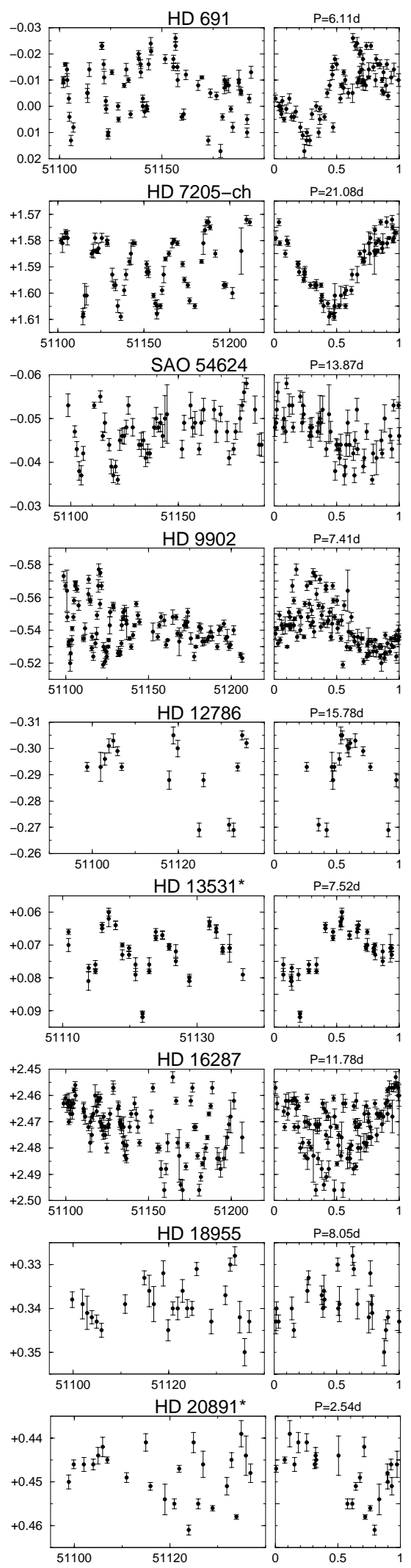
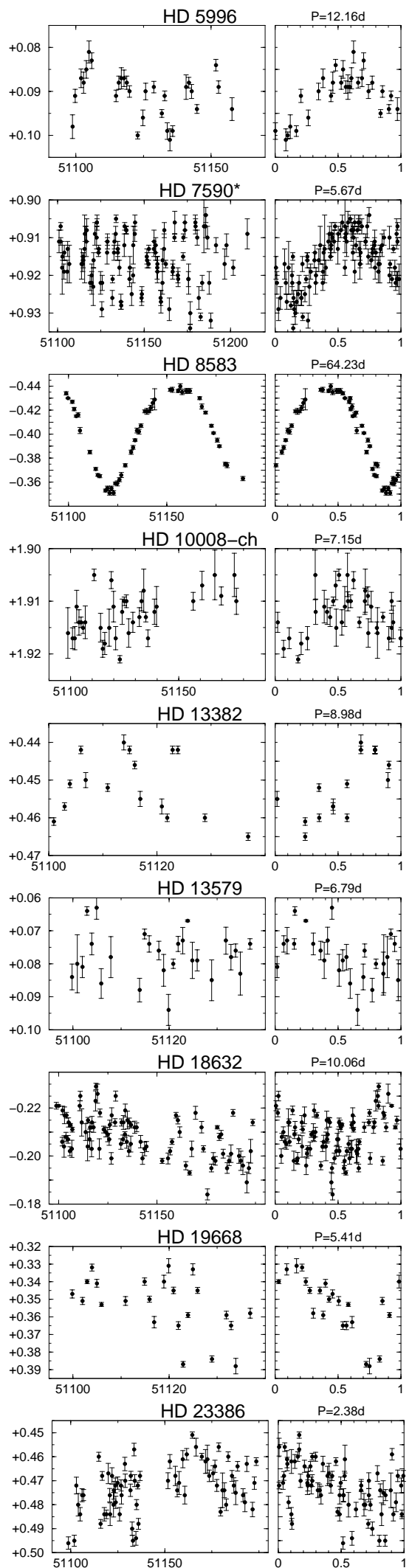

Fig. B4. Differential Strömgren-y light curves of stars with Ca II H and K emission. The HD number (or HIP number, or Variable Star designation, if no HD number exists) is identified on top of each plot. The left panel in each plot is the observations versus heliocentric Julian date. The right panel is the light curve phased with the best-fit photometric period from Table A3. Error bars for the observations indicate the variances from three individual readings between the variable and the comparison star 
K.G. Strassmeier et al.: The Vienna-KPNO search for Doppler-imaging candidate stars. I.
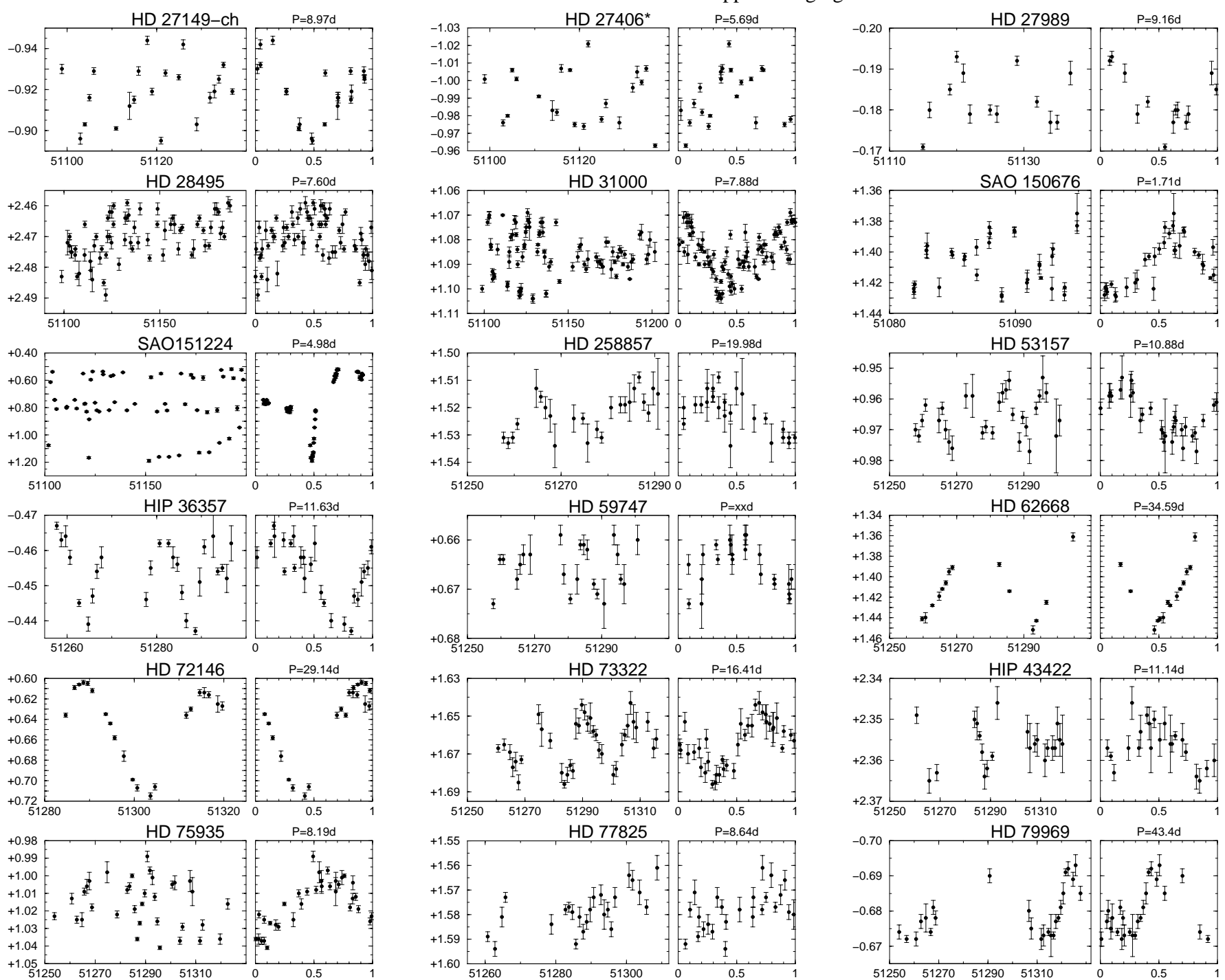

\begin{tabular}{cccccc}
+1.051250 & 51270 & 51290 & 51310 & 0 & 0.5 \\
& & HD 82159 & & $P=3.04 d$ \\
\hline
\end{tabular}

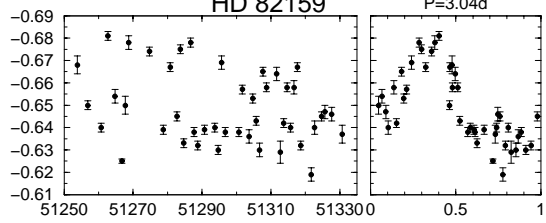

$\begin{array}{rrrrrr}-0.61 & 1 & 1 & 1 & 1 & 1 \\ 51250 & 51270 & 51290 & 51310 & 513300 & 0.5 \\ & & & H D & 83983 & P=10.92 d\end{array}$

+1.62
+1.63
+1.64
+1.65

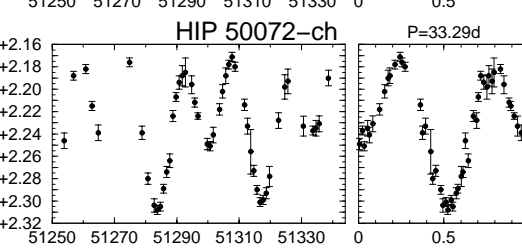

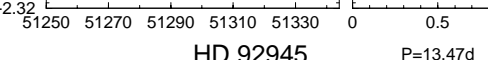
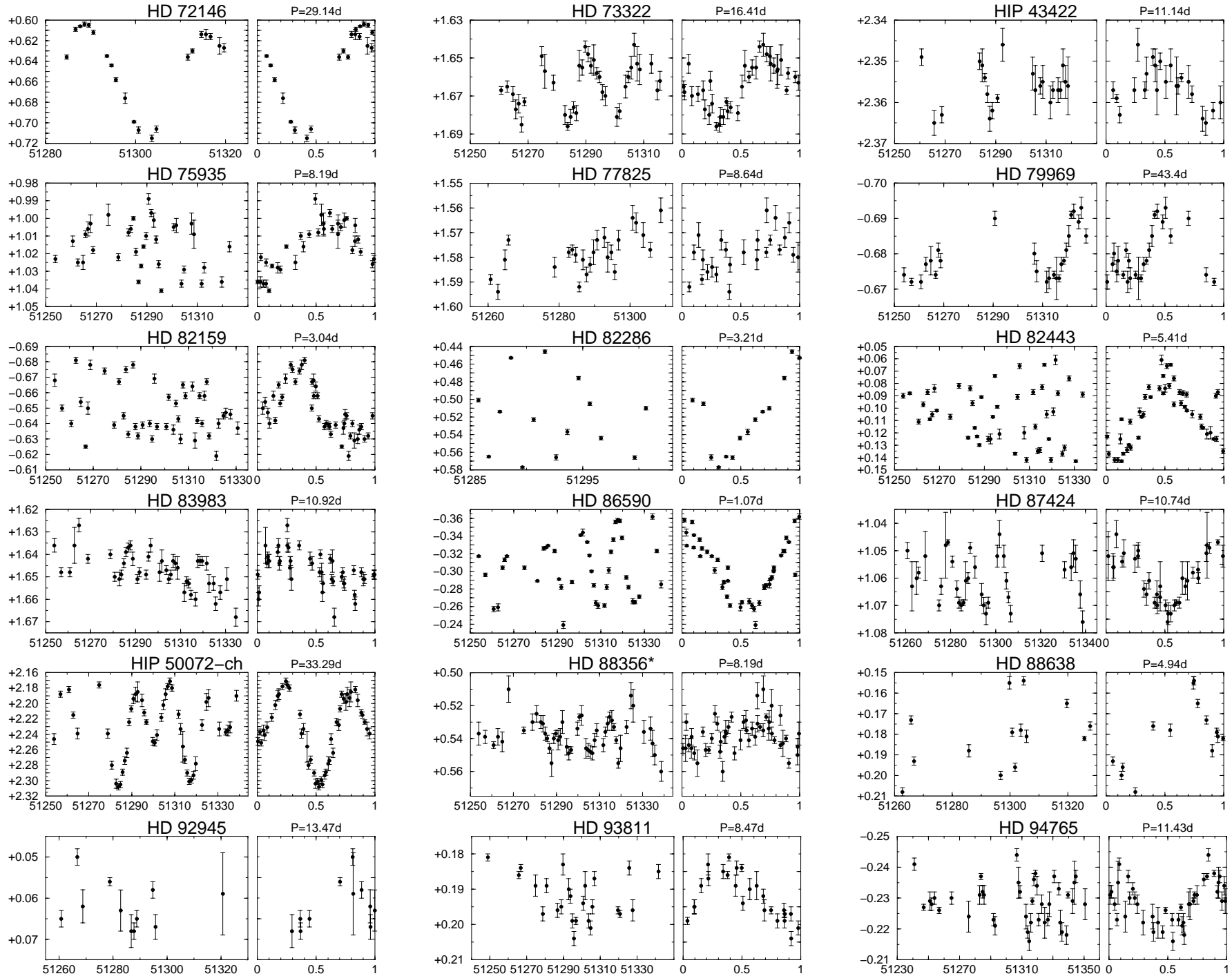

Fig. B4. continued 
K.G. Strassmeier et al.: The Vienna-KPNO search for Doppler-imaging candidate stars. I.

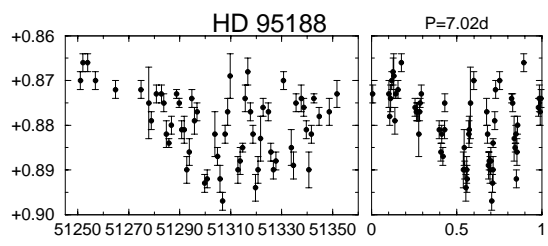

HD 95743

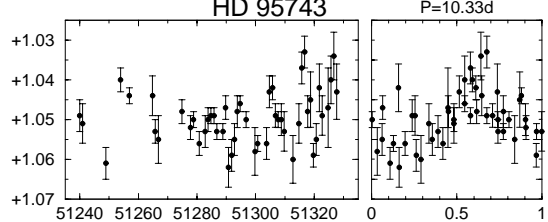

$\begin{array}{rrrr}+1.07 & \\ 5124051260512805130051320 & 0 & 0.5 \\ & \text { HIP } 57859 & P=11.43 d\end{array}$
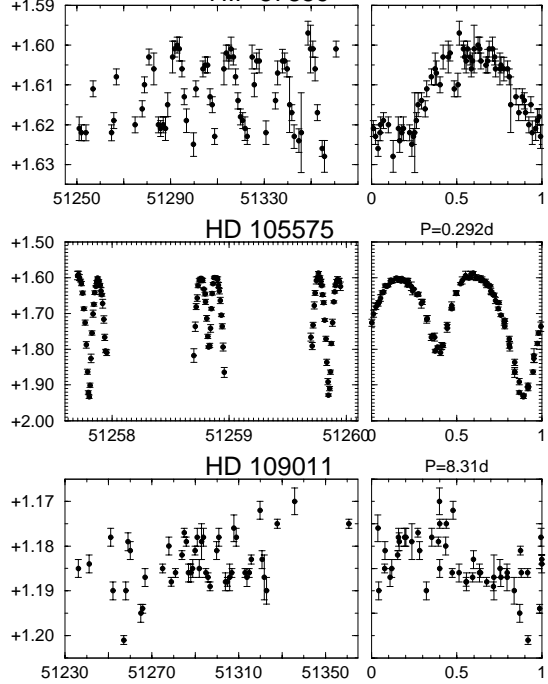

+1.35 HD $110463 \quad P=11.75 d$
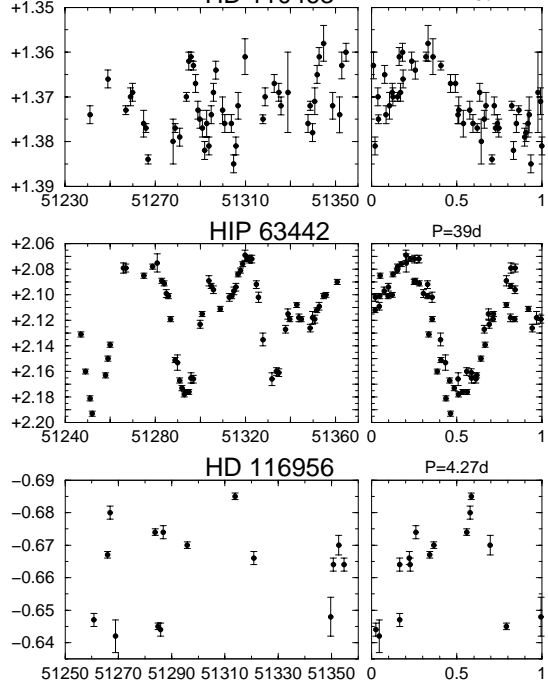

$+0.83 \quad$ HD $125874 \quad P=7.52 \mathrm{~d}$
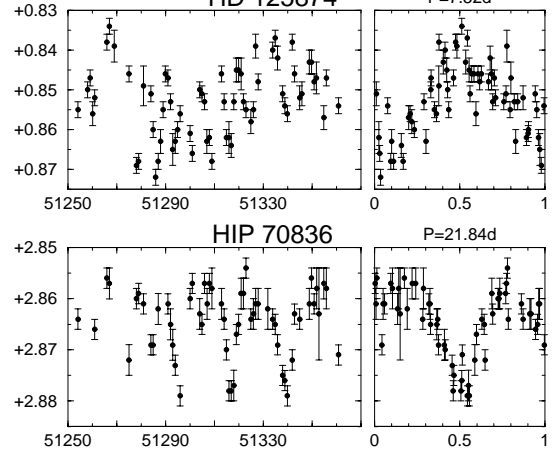

HD 95559
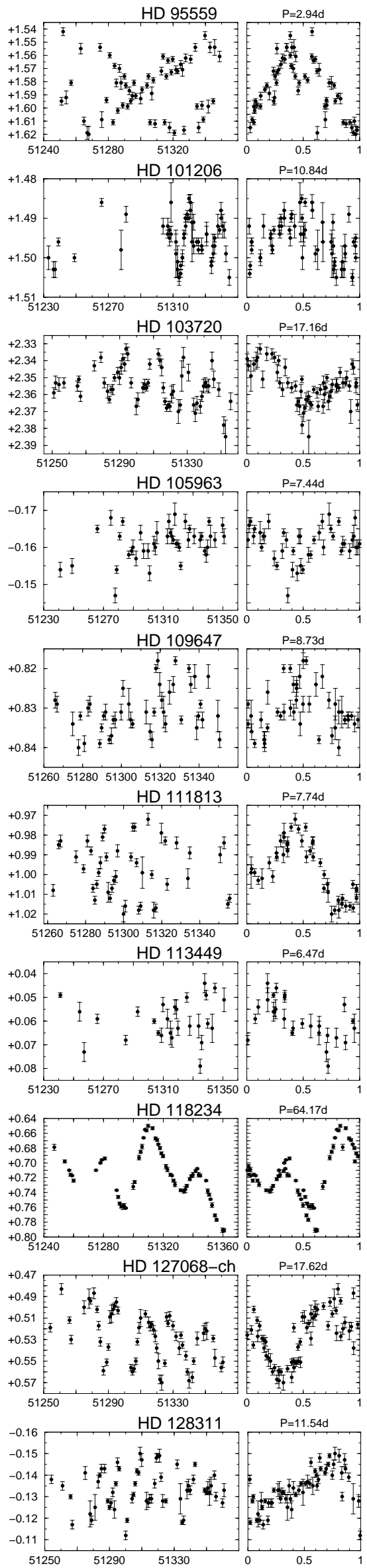
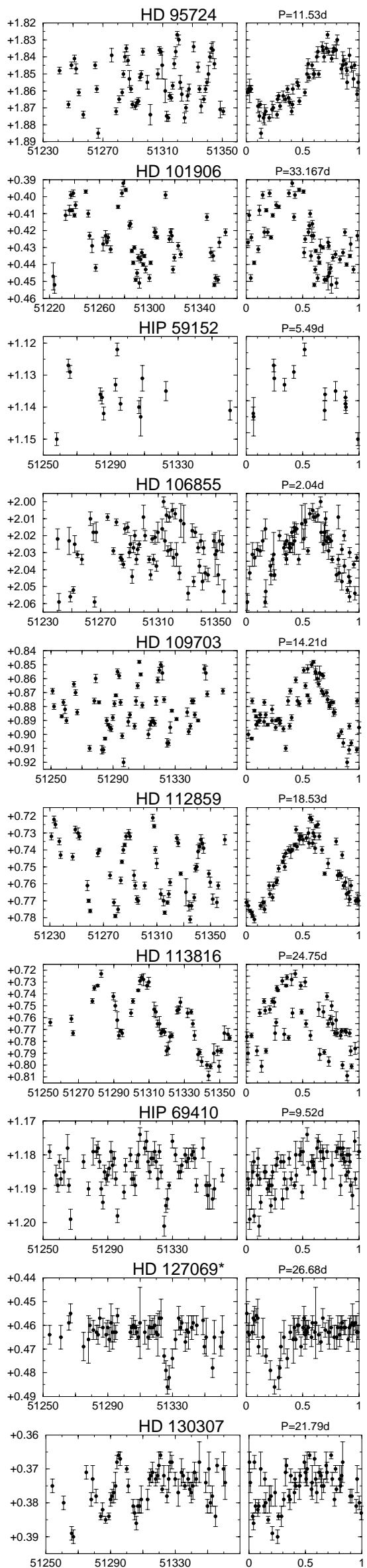

Fig. B4. continued 
K.G. Strassmeier et al.: The Vienna-KPNO search for Doppler-imaging candidate stars. I.
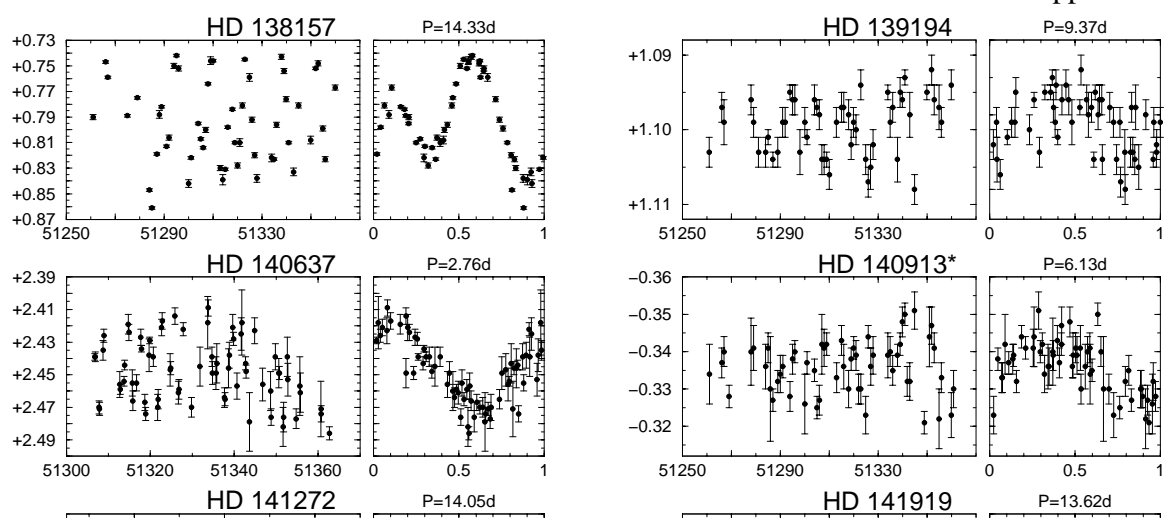

+0.87
+0.88
+0.89
+0.90
+0.91
+0.92
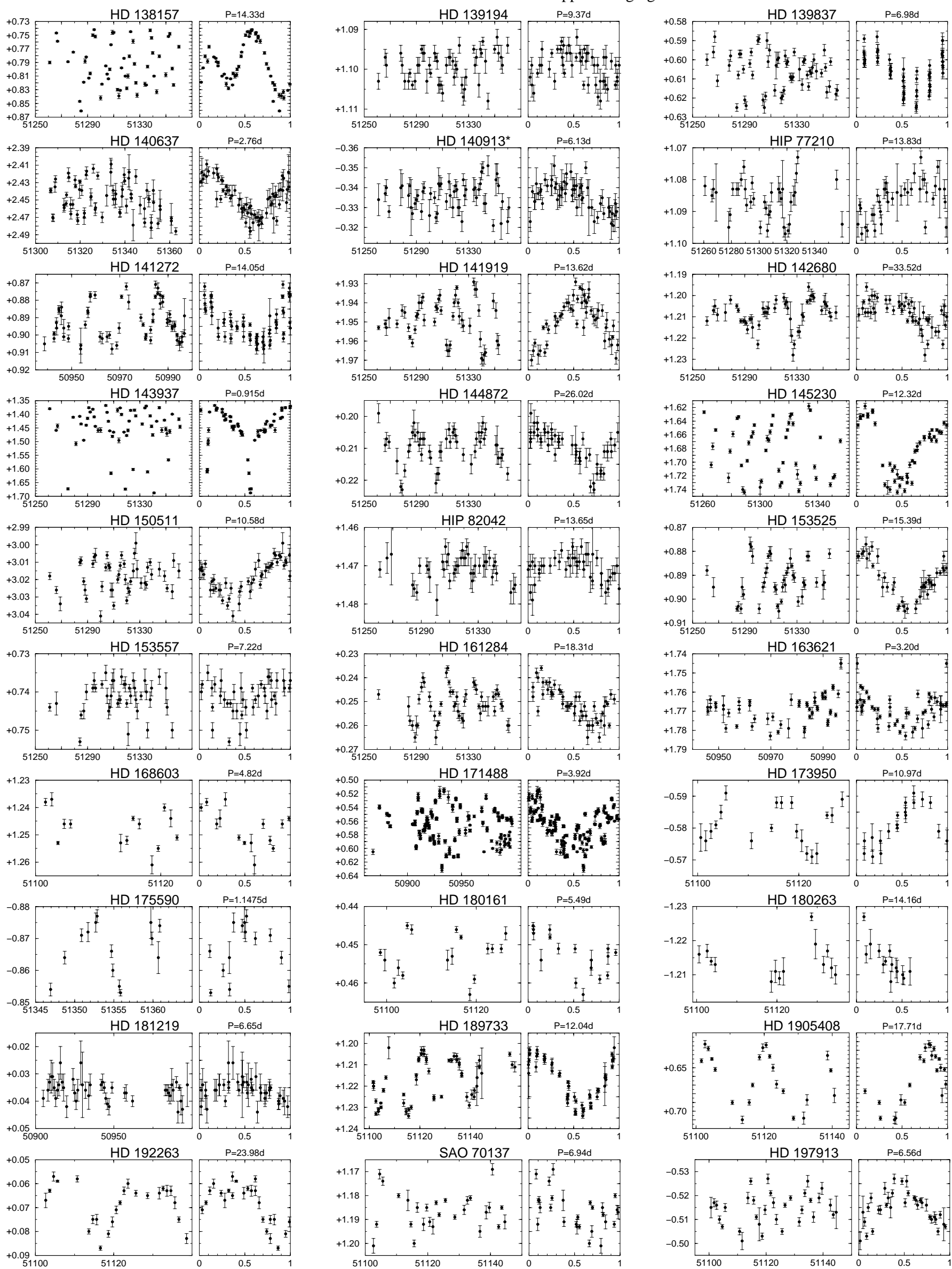

Fig. B4. continued 
K.G. Strassmeier et al.: The Vienna-KPNO search for Doppler-imaging candidate stars. I.
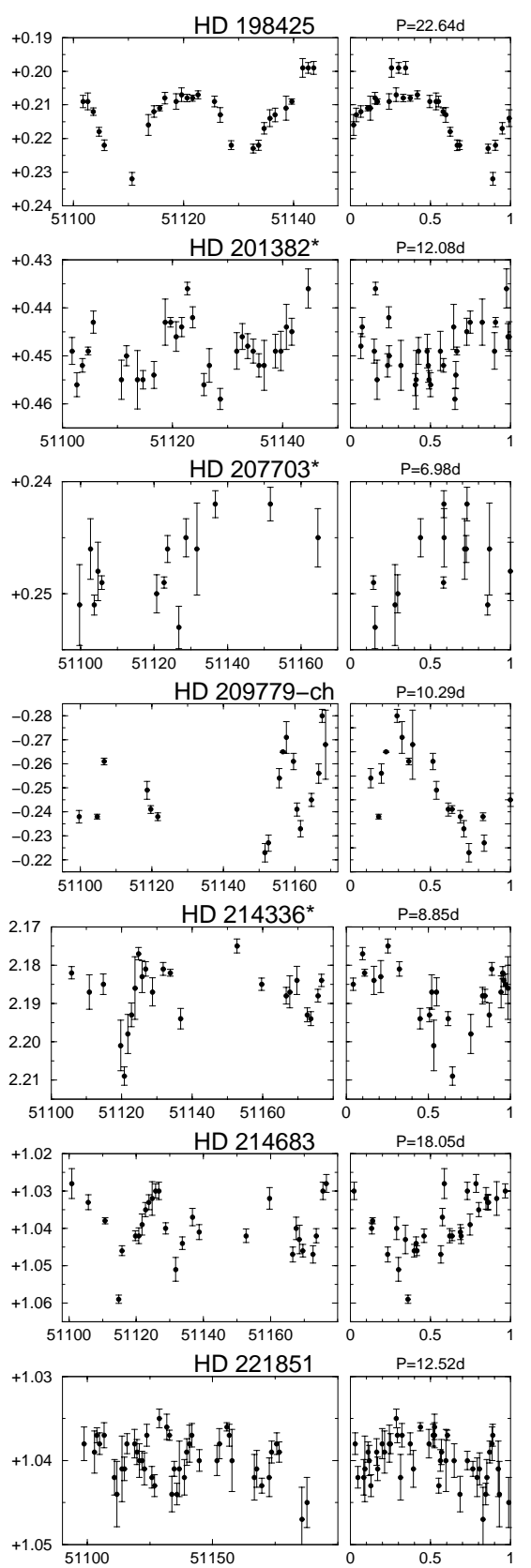

Fig. B4. continued
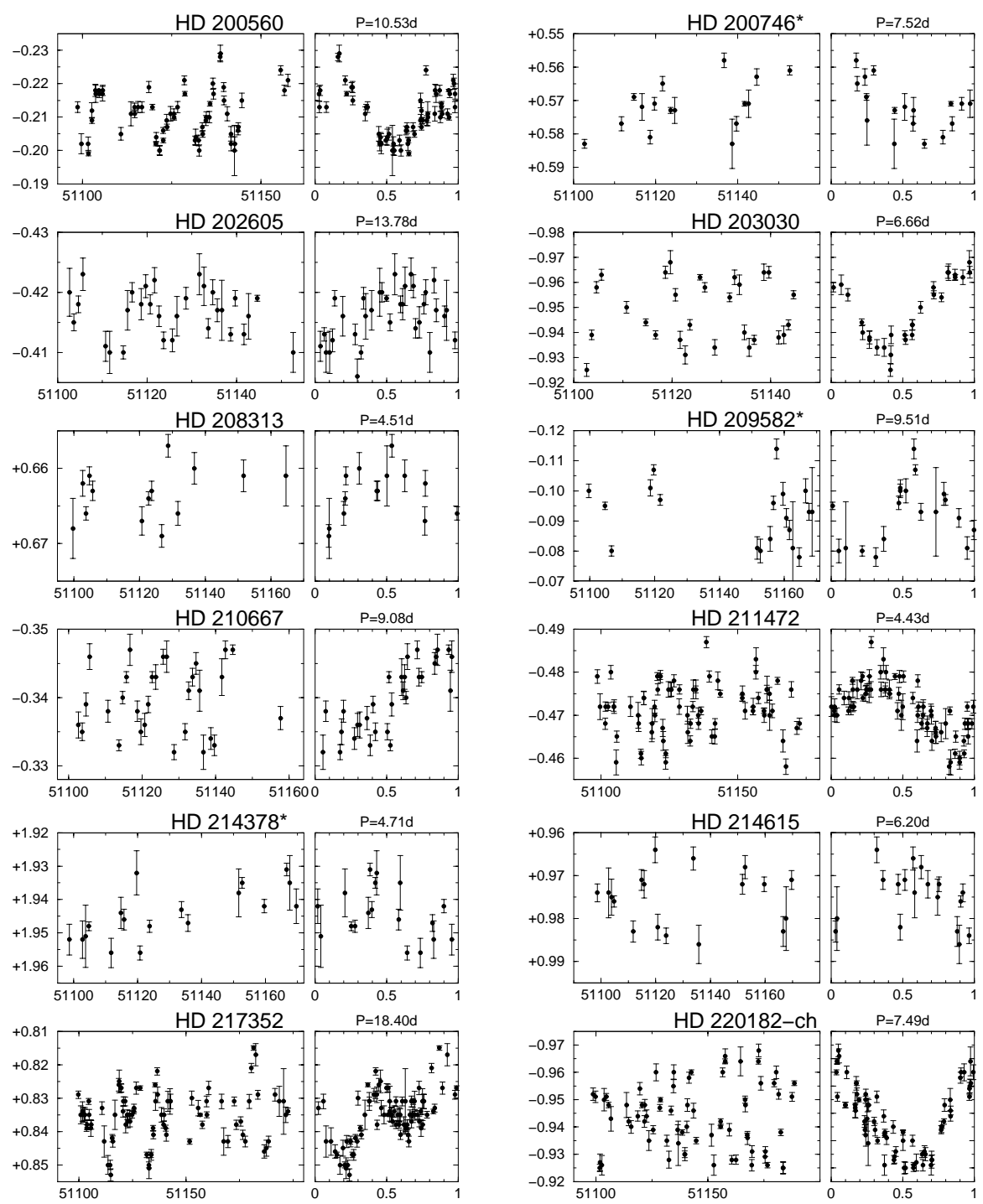
HD 220182-ch $P=7.49 d$

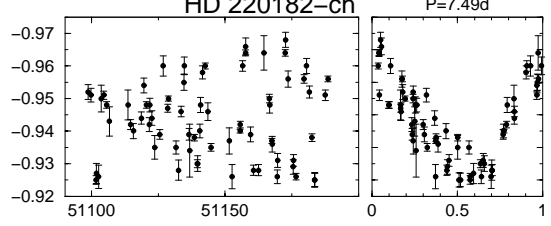


K.G. Strassmeier et al.: The Vienna-KPNO search for Doppler-imaging candidate stars. I.
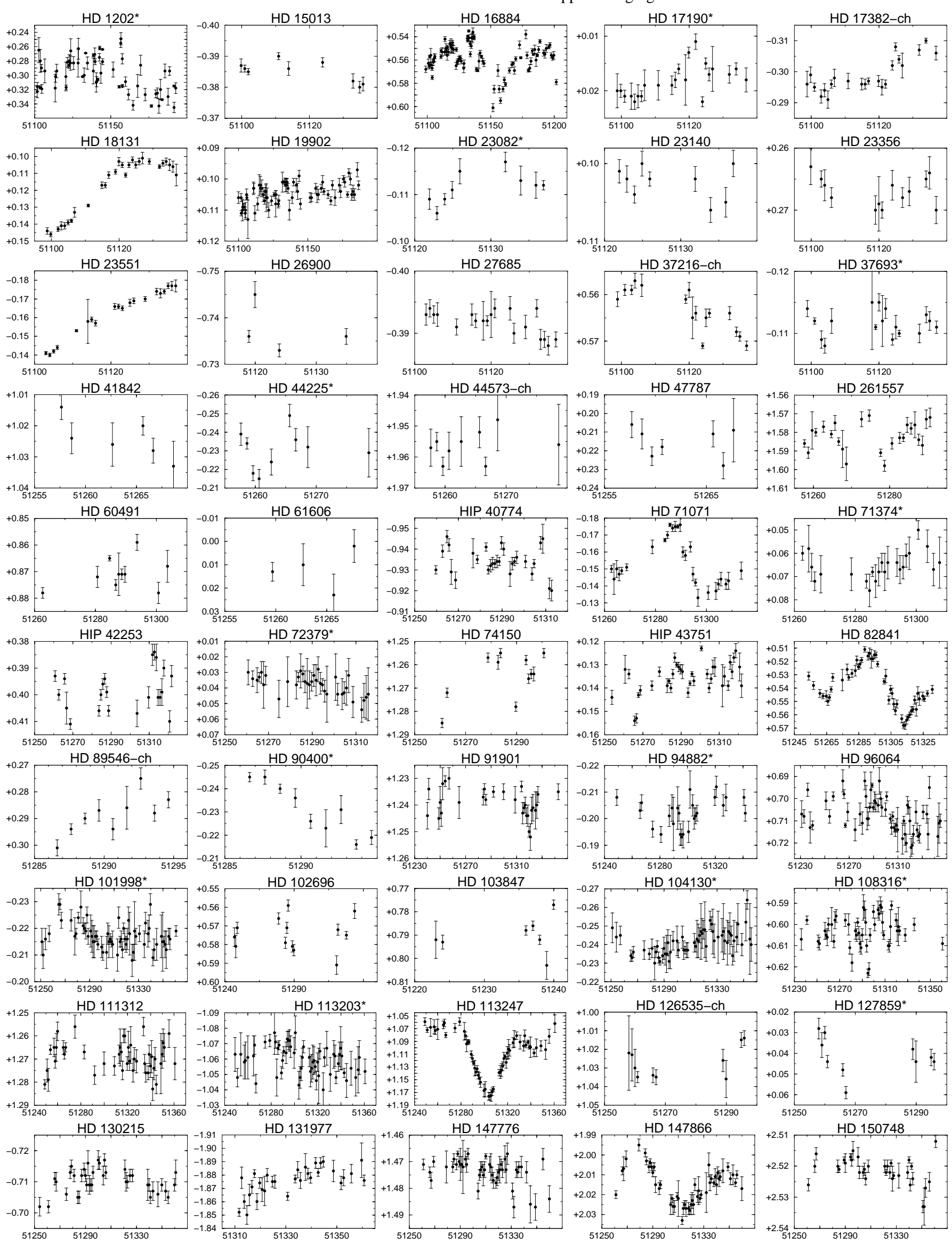

Fig. B5. Differential Strömgren-y light curves of stars without a period determination. Note that many of these stars are likely photometric variable but the time coverage was just too short to cover a full cycle. Otherwise as in Fig. B4 
K.G. Strassmeier et al.: The Vienna-KPNO search for Doppler-imaging candidate stars. I.
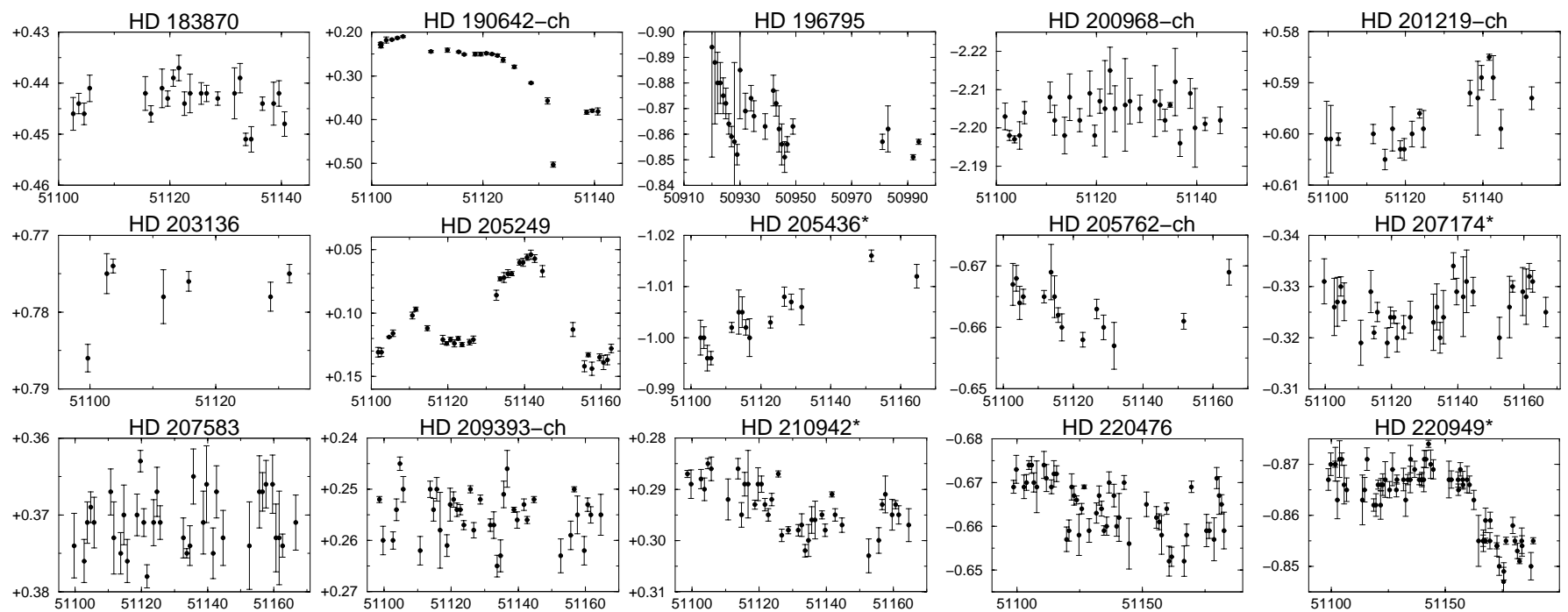

HD 220949

Fig. B5. continued 